

\title{
Graph Factors and Matching Extensions
}

\author{
Qinglin Roger $\mathrm{Yu}^{13}$ and Guizhen $\mathrm{Liu}^{2}$ \\ 1. Department of Mathematics \& Statistics \\ Thompson Rivers University, Kamloops, BC, Canada \\ and \\ 2. School of Mathematics \\ Shandong University, Jinan, P. R. China \\ and \\ 3. Center for Combinatorics \\ Nankai University, Tianjin, P. R. China
}

October 25, 2008

\footnotetext{
${ }^{1}$ Supported by Natural Sciences and Engineering Research Council of Canada under grant OGP0122059, and NSFC 60673047
} 


\section{Contents}

1 Matchings and Perfect Matchings 1

1.1 Definitions and terminologies .............. . . 3

1.2 Matchings in bipartite graphs and augmenting path . . . . . . . 6

1.3 Matchings in non-bipartite graphs . . . . . . . . . . . . . . . . 11

1.4 Sufficient conditions for 1-factors . . . . . . . . . . . 15

1.5 Gallai-Edmonds Structure Theorem . . . . . . . . . . . . . . 24

1.6 Number of 1 -factors . . . . . . . . . . . . . . . . . 33 


\section{Chapter 1}

\section{Matchings and Perfect Matchings}

As a young branch of mathematics, Graph Theory has experienced the explosion growth as the same phenomenon was taking place in computing science and communication networking. In the mean time, there are many new terminologies and knowledge accumulated in the process. So naturally, often there are more than one names or notions defined for a same entity. So we start to list the most terms and notions used in this book and hope to provide the readers with a consistent reference.

\section{Notions}

Let $G$ be a graph, with vertex set $V(G)$ and edge set $E(G)$. Let $f$ be a positive integer-valued function defined on vertex set $V(G)$.

$|S|$ : order of a set $S$;

$\lfloor x\rfloor$ : the largest integer not greater than $x$;

$\lceil x\rceil$ : the smallest integer not less than $x$;

$V(G)$ : the vertex set of $G$, sometimes, we use $|G|$ for the order of G, i.e., $|G|=$ $|V(G)|$

$E(G)$ : the edge set of $G$; occasionally we write $\|G\|$ for the size of $G$, i.e., $\|G\|=$ $|E(G)|$

$A \triangle B$ : symmetric difference of two sets $A$ and $B$, i.e., $A \triangle B=(A-B) \cup(B-A)$;

$d_{G}(v)$ : the degree of a vertex $v$ in $G$;

$\delta(G)$ : the minimum degree of $G$;

$\Delta(G)$ : the maximum degree of $G$;

$\nabla(v)$ : the edges incident with the vertex $v$;

$\nabla(S)$ : the set of edges with exactly one end-vertex in the set $S$;

$N_{G}(S)$ : the set of vertices adjacent to a vertex of $S$, i.e., $N_{G}(S)=\{x \mid x y \in E(G)$ and $y \in S\}$;

$E_{G}(S, T)$ : the edges with one end in $S$ and another end in $T$ and $e_{G}(S, T)=$ $\left|E_{G}(S, T)\right|$ 
$\sigma_{k}(G)$ : degree sum of $k$ independent vertices;

$\mu(G)$ : the matching number or fractional matching number of $G$;

$\eta(G)$ : maximum cardinality of a join of $G$;

$\xi(\Sigma)$ : the smallest integer such that no graph $G$ embeddable in surface $\Sigma$ is $\xi(\Sigma)$ extendable;

$\operatorname{ext}(G)$ : extendability number of a graph $G$;

$b(G)$ : the number of bricks of $G$ in a decomposition;

$\hat{c}(G)$ : the number of critical $\left\{K_{2}, K_{3}\right\}$-components in $G$

$p(G)$ : the number of Petersen graphs;

$i \mu(G)$ : the induced matching number;

$G^{c}$ : the complement of $G$;

$G[S]$ : the induced subgraph of $G$ by a vertex set $S$;

$o(G)$ : the number of odd components of $G$;

$\omega(G)$ : the number of components of $G$;

$c(G)$ : the circumference of $G$;

$\beta^{*}(G)$ : the vertex covering number of $G$;

$I(G)$ : the set of isolated vertices in $G$;

$i(G)$ : the number of isolated vertices in $G$;

$D(G)$ : the set of all vertices in $G$ which are not saturated by at least one maximum matching of $G$;

$A(G)$ : the set of vertices in $V(G)-D(G)$ adjacent to at least one vertex in $D(G)$; $C(G)=V(G)-D(G)-A(G)$

$f c(G)$ : the number of factor-critical components in $G$;

$\operatorname{tc}(G)$ : the number of triangle clusters in $G$;

$o c(G-S)$ : the number of those components of $G-S$ that are odd cacti;

$\Phi(G)$ : the number of 1 -factors in $G$;

$G_{\delta}$ : the subgraph induced by the vertices with minimum degree in $G$;

$G_{\Delta}$ : the subgraph induced by the vertices with maximum degree in $G$;

$\kappa(G)$ : the vertex connectivity of $G$;

$\kappa^{\prime}(G)$ : the edge connectivity of $G$;

$\lambda(C)$ : characteristic of a cut $C$;

$c \lambda(G)$ : the cyclic connectivity of $G$;

$\alpha(G)$ : independent number of $G$;

$\gamma(G)$ : the dominating number;

$\gamma^{*}(G)$ : the genus of $G$;

$\chi^{\prime}(G)$ : the edge chromatic number of $G$;

$\chi_{c}^{\prime}(G)$ : the edge-cover number of $G$;

$\tau_{2}(G)$ : the size of a minimum 2-covering of $G$;

$G \vee H$ : the join of two graphs $G$ and $H$;

$G^{r}$ : the $r$ th power of a graph $G$. 
$t(G)$ : toughness of $G$ introduced by Chvátal [153],

$$
t(G)=\min \left\{\frac{|S|}{\omega(G-S)} \mid S \subseteq V(G), \omega(G-S) \geq 2\right\}
$$

$\tau(G)$ : a variation of toughness introduced by Enomoto [187],

$$
\tau(G)=\min \left\{\frac{|S|}{\omega(G-S)-1} \mid S \subseteq V(G), \omega(G-S) \geq 2\right\}
$$

We define, for all disjoint subsets $S$ and $T$ of $V(G)$,

$$
\delta_{G}(S, T)= \begin{cases}k|S|+\sum_{x \in T} d_{G}(x)-k|T|-e_{G}(S, T)-q_{G}(S, T) & \text { for } k \text {-factor; } \\ f(S)+\sum_{x \in T} d_{G}(x)-f(T)-e_{G}(S, T)-q_{G}(S, T) & \text { for } f \text {-factor; } \\ f(S)+\sum_{x \in T} d_{G}(x)-g(T)-e_{G}(S, T)-q_{G}(S, T) & \text { for }(g, f) \text {-factor. }\end{cases}
$$

\subsection{Definitions and terminologies}

To understand the developments of matchings and factors in graphs, we would like to review the history briefly by listing the most significant milestones in this fascinating area of graph theory.

The first attempt on the study of factors was due to Danish mathematician Petersen (1891), who proved that every graph of even degrees can be decomposed into the union of edge-disjoint 2-factors. This was motivated from the study of an algebraic factorization problem. He also showed that every 2-connected cubic graph has a 1-factor. These two results can be viewed as a forerunner of modern graph factor theory.

For matchings in bipartite graphs, König (1931) and Hall (1935) obtained the so-called König-Hall Theorem (sometimes, it is known as Hall's Theorem). Due to its wide applications to many graph theory problems and to other branches of mathematics, König-Hall Theorem remains one of most influential graph-theoretic results.

In 1947, Tutte gave a characterization (i.e, so-called Tutte's 1-Factor Theorem) for the existence of perfect matchings in arbitrary graphs and it has become a cornerstone of factor theory. Till now, this elegant theorem is still one of the most fundamental results in factor theory. Subsequently, Tutte (1952) extended the techniques in the proof of 1-Factor Theorem to obtain a sufficient and necessary condition for a graph to have an $f$-factor.

Gallai (1964) and Edmonds (1965), independently, investigated a canonical decomposition of arbitrary graphs in terms of its maximum matchings and thus revealed the structure of graphs related to matchings. This deep and important result is referred as Gallai-Edmonds Structure Theorem. The existence of the canonical decomposition 
and an efficient method to find such a decomposition allowed Edmonds to obtain the first polynomial algorithm for finding maximum matchings in graphs.

The most general degree-constrained factors, $(g, f)$-factors, were studied by Lovász (1970). He gave necessary and sufficient conditions for a graph to have a $(g, f)$-factor. This theorem generalized the criteria of all other factors, such as 1 -factors, $k$-factors, $f$-factors and $[a, b]$-factors.

For the more comprehensive account of history on matching theory and graph factors, readers can refer to Preface of Lovász and Plummer's Matching Theory or Biggs, Lloyd and Wilson's Graph Theory 1736-1936.

In this book, we will mainly deal with factors in finite undirected simple graphs. Some results also hold for graphs with multiple edges, in such cases, we will point out.

The degree of a vertex $v$ in a graph $G$, denoted by $d_{G}(v)$, is the number of edges of $G$ incident with $v$, each loop counting as two edges. In particular, if $G$ is a simple graph, $d_{G}(v)$ is the number of neighbours of $v$ in $G$. A vertex of degree zero or one are called an isolated vertex and a leave, respectively. We denote by $\delta(G)$ and $\Delta(G)$ the minimum and maximum degrees of the vertices of $G$.

A cut-edge of a graph is one whose deletion results in one more component. Sometimes, a cut-edge is also called a bridge.

A matching $M$ of a graph $G$ is a subset of $E(G)$ such that any two edges of $M$ have no end-vertices in common. A matching of $k$ edges is called a $k$-matching. Let $d$ be a non-negative integer. A matching is called a defect-d matching if it covers exactly $|V(G)|-d$ vertices of $G$. A defect-0 matching is called a perfect matching and defect-1 matching is called near-perfect matching.

A perfect matching is also referred as 1-factor since it is a 1-regular subgraph. If a graph has 1-factors, it is called 1-factorable. There are probably equal numbers of people using "perfect matchings" rather than "1-factors", so we will use them non-discriminatively.

If an edge $e$ is contained in a 1-factor, we called $e$ an allowed edge. If an edge $f$ does not lie in any 1-factor, then $f$ is forbidden.

A covering of a graph $G$ is a subset $S$ of $V(G)$ such that every edge of $G$ has at least one end in $S$. A covering $S^{*}$ is a minimum covering if $G$ has no covering $S$ with $|S|<\left|S^{*}\right|$. The order of a minimum covering is called covering number and denoted by $\beta^{*}(G)$.

A graph $G$ is said to be bicritical if for every pair of distinct vertices $u$ and $v$, $G-\{u, v\}$ has a 1-factor. A graph $\mathrm{G}$ is factor-critical if $G-u$ has a 1-factor for every $u \in V(G)$.

A graph $G$ is called $n$-factor-critical if the subgraph $G-S$ has a 1 -factor for all $n$-subset $S$ of $V(G)$.

Clearly, the concept of $n$-factor-criticality is a generalization of bicriticality and factor-criticality. That is, bicritical graphs and factor-critical graphs are $n$-factor- 


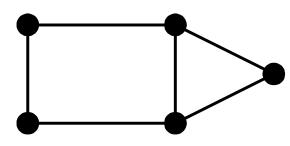

(a)

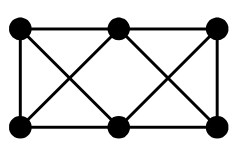

(b)

Figure 1.1: (a) a factor-critical graph and (b) a bicritical graph

critical graph when $n=1$ and 2 , respectively.

From the definitions, we can see the following result easily.

Proposition 1.1.1 (a) A graph $G$ is factor-critical if and only if the join of $G$ and $K_{1}, G \vee K_{1}$, is bicritical;

(b) a graph $G$ is bicritical if and only if $G-v$ is factor-critical for every $v \in V(G)$.

Let $G$ be a connected graph with a 1-factor and $|V(G)| \geq 2 k+2$. If each $k$ matching of $G$ is contained in a 1 -factor, we call $G$ a $k$-extendable graph. For convenience, a 0-extendable graph means a graph which has a 1-factor.

Clearly bicritical graphs are 1-extendable. A 3-connected bicritical graph is called a brick. A brace is a 2-extendable bipartite graph (i.e., 3-connected 2-extendable bipartite graph).

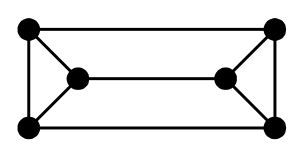

(a)

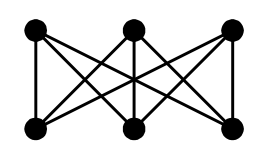

(b)

Figure 1.2: (a) a brick and (b) a brace

Factors in a graph is spanning subgraphs satisfying some given properties. A $k$-factor of a graph $G$ is a spanning subgraph $H$ such that $d_{H}(x)=k$ for every $x \in V(G)$.

Let $f$ be an integer-valued function defined on $V(G)$, i.e.,

$$
f: V(G) \rightarrow \mathbb{Z}^{+}=\{0,1,2, \ldots\},
$$

then a spanning subgraph $H$ such that $d_{H}(x)=f(x)$ for all $x \in V(G)$ is called an f-factor.

A more general factor is so-called $(g, f)$-factors: for two functions $g, f: V(G) \rightarrow$ $\mathbb{Z}$, a spanning subgraph $H$ is called a $(g, f)$-factor if $g(x) \leq d_{H}(x) \leq f(x)$ for all $x \in V(H)$. In addition, if $g(x) \equiv f(x)(\bmod 2)$ for all $x \in V(G)$, a $(g, f)$-factor $F$ with $d_{F}(x) \equiv f(x)$ is called a $(g, f)$-parity-factor. 


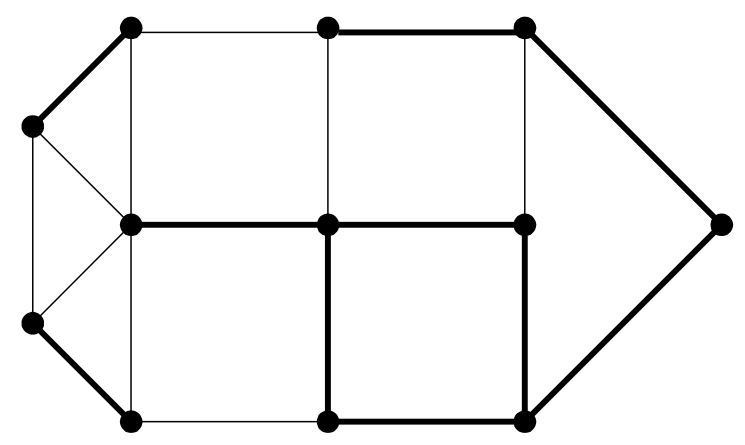

Figure 1.3: When $g(x)=1, f(x)=3$, a spanning subgraph $H$ is a $(g, f)$-factor

For two positive integers $a, b$, let $g(x)=a$ and $f(x)=b$ for all vertex $x \in V$, then $(g, f)$-factors are called $[a, b]$-factors.

A set $S \subset V$ is a dominating set of $G$ if every vertex in $V$ is either in $S$ or is adjacent to a vertex in $S$. If $A$ and $B$ are subsets of $V$, we say that $A$ dominates $B$ if every vertex of $B$ has a neighbor in $A$ or is a vertex of $A$; we also say that $B$ is dominated by $A$. The dominating number, $\gamma(G)$, of $G$ is the minimum cardinality of a dominating set. A set $S$ is 2-dominating if every vertex of $V$ is either in $S$ or is within distance 2 to $S$.

We only define the most often used terms of the book in this section. For other undefined terms, we follow Bollobás [85].

\subsection{Matchings in bipartite graphs and augmenting path}

In this section, we focus on matchings and perfect matchings in bipartite graphs. From historic point of view, it is the starting point of matching theory. This is not surprise since matchings in bipartite graphs are closely related to other disciples of mathematics (e.g., matrix theory and set theory) and to many application problems (e.g., the job assignment problem). Furthermore, the properties and techniques of matchings in bipartite graphs also motivate the research of matchings in non-bipartite graphs.

For a matching $M$, a vertex $x$ of $G$ is called saturated or covered by $M$ if $x$ is incident to an edge of $M$. A matching $M$ is called a maximum matching if $|M| \geq\left|M^{\prime}\right|$ for any matching $M^{\prime}$ of $G$. Clearly, a perfect matching (i.e., a 1-factor) is a maximum matching saturating every vertex of a graph. Of course, any graph with perfect matchings must have even number of vertices.

In many applications, one wishes to find a matching in a bipartite graph $G=$ 
$(X, Y)$ which covers every vertex in $X$. Necessary and sufficient conditions for the existence of such a matching were proven by Hall (1935). To this day, Hall's Theorem remains one of most applied graph-theoretic results. The proof here is due to Rado.

Theorem 1.2.1 (Hall's Theorem, Hall (1935)) Let $G=(X, Y)$ be a bipartite graph. Then $G$ has a matching saturating all vertices of $X$ if and only if for any $S \subseteq X$,

$$
|N(S)| \geq|S| .
$$

Proof. The necessity is clear.

Let $G$ be a minimal bipartite graph satisfying the condition (1.1). It suffices to show that $G$ consists of $|X|$ independent edges.

Otherwise, $G$ contains two edges, $x_{1} y$ and $x_{2} y$, where $x_{1}, x_{2} \in X, x_{1} \neq x_{2}$ and $y \in Y$. From the minimality, the deletion of either of these edges invalidates (1.1). Thus there are two subsets $X_{1}, X_{2} \subset X$ such that for $i=1,2$ we have $\left|N\left(X_{i}\right)\right|=\left|X_{i}\right|$, and $x_{i}$ is the only vertex of $X_{i}$ adjacent to $y$. Then

$$
\begin{aligned}
\left|N\left(X_{1}\right) \cap N\left(X_{2}\right)\right| & \geq\left|N\left(X_{1}-\left\{x_{1}\right\}\right) \cap N\left(X_{2}-\left\{x_{2}\right\}\right)\right|+1 \\
& \geq\left|N\left(X_{1} \cap X_{2}\right)\right|+1 \geq\left|X_{1} \cap X_{2}\right|+1 .
\end{aligned}
$$

But this implies the following contradiction:

$$
\begin{aligned}
\left|N\left(X_{1} \cup X_{2}\right)\right| & =\left|N\left(X_{1}\right) \cup N\left(X_{2}\right)\right| \\
& =\left|N\left(X_{1}\right)\right|+\left|N\left(X_{2}\right)\right|-\left|N\left(X_{1}\right) \cap N\left(X_{2}\right)\right| \\
& \leq\left|X_{1}\right|+\left|X_{2}\right|-\left|X_{1} \cap X_{2}\right|-1 \\
& =\left|X_{1} \cup X_{2}\right|-1 .
\end{aligned}
$$

Note that Hall's Theorem also holds for multigraphs. The above result is the usual version of matching characterization in bipartite graphs. However, we present the next version in terms of isolated vertices, which has a strong similarity to Tutte's 1-Factor Theorem appeared later. Recall that $i(G)$ denotes the number of isolated vertices in $G$.

Theorem 1.2.2 (Hall's Matching Theorem, Hall (1935)) Let $G=(X, Y)$ be a bipartite graph. Then $G$ has a perfect matching if and only if $|X|=|Y|$ and for any $S \subseteq X$

$$
i(G-S) \leq|S| .
$$

Remark 1.2.1. For every bipartite graph $G=(X, Y)$, with $|X| \geq|Y|$ and maximum degree $\Delta$, König (1916) showed that there exists $\Delta$-regular bipartite super-graph $G^{\prime}=\left(X, Y^{\prime}\right)$ (a multigraph is allowed) such that $Y \subseteq Y^{\prime}$ and $G$ is a subgraph of $G^{\prime}$. 
Corollary 1.2.1 (König (1916)) Every r-regular bipartite graph $G$ can be decomposed into $r$ edge-disjoint 1-factors. In particular, $G$ has a 1-factor.

Corollary 1.2.2 Every bipartite graph $G$ has a maximum matching which covers all the vertices of maximum degree.

Proof. By Remark 1.2.1, there exists a $\Delta(G)$-regular supergraph $G^{\prime}$ containing $G$. From Corollary 1.2.1, $G^{\prime}$ has a 1 -factor and so $G$ has a matching covers all the vertices of maximum degree. In turn, this matching can be augmented to a maximum matching of $G$.

A family of graphs which share many properties with bipartite graphs was discovered by Fulkerson, Hoffman and McAndrew [221]. A graph $G$ is said to have the odd cycle property (OCP in short) if every pair of odd cycles in $G$ either have a vertex in common or are joined by an edge. We shall discuss the relation between this property and the existence of factors in much more details in Section 2.2.

The following is a generalization of Corollary 1.2.1.

Theorem 1.2.3 If $G$ is a r-regular graph of even order with the odd cycle property, then $G$ has a 1-factor.

Let $M$ be a matching in a graph $G$. An $M$-alternating path or cycle in $G$ is a path or cycle whose edges are alternately in $M$ and $E(G)-M$. Note that an $M$-alternating path might or might not start or end with edges of $M$. If neither its origin nor its terminus is covered by $M$, the $M$-alternating path is called an $M$-augmenting path.

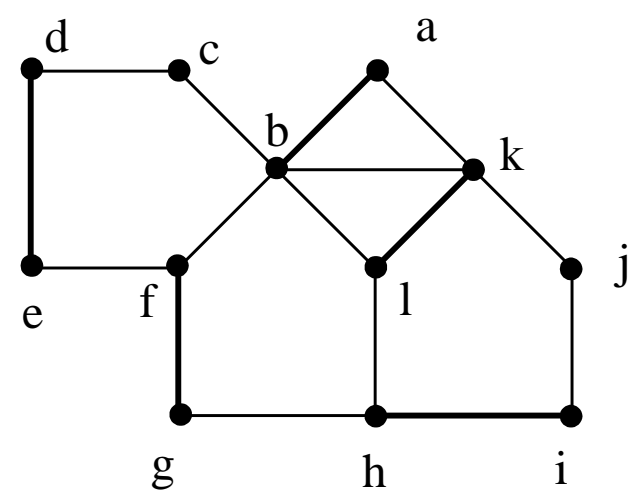

Figure 1.4: An $M$-alternating cycle $C=a b l k$, an $M$-alternating path $P=\operatorname{defghi}$ and an $M$-augmenting path $P^{\prime}=$ cdefghij.

The idea of $M$-augmenting path is first introduced by Berge (1957). Today, it has become a very matured and standard technique in the study of factor theory. There are many variations of augmenting path defined for different problems, we 
shall see all kinds of variations in the coming chapters. It is not an overstatement that augmenting path technique is the most powerful technique not only in the study of matchings and also for the study of all factors.

Berge (1957) use augmenting path to give a characterization of maximum matching.

Theorem 1.2.4 (Berge (1957)) A matching $M$ in a graph $G$ is maximum if and only if $G$ has no $M$-augmenting path.

Proof. Let $M$ be a matching in $G$. Suppose that $G$ contains an $M$-augmenting path $P$. Then $M^{\prime}:=M \triangle E(P)$ is a matching in $G$, and $\left|M^{\prime}\right|=|M|+1$. Thus $M$ is not a maximum matching.

Conversely, suppose that $M$ is not a maximum matching, and let $M^{\prime \prime}$ be a maximum matching in $G$, so that $\left|M^{\prime \prime}\right|>|M|$. Set $H:=G\left[M \triangle M^{\prime \prime}\right]$. Then each vertex of $H$ has degree one or two in $H$, for it can be incident with at most one edge of $M$ and one edge of $M^{\prime \prime}$. Consequently, each component of $H$ is either an even cycle with edges alternately in $M$ and $M^{\prime \prime}$, or else a path with edges alternately in $M$ and $M^{\prime \prime}$.

Because $\left|M^{\prime}\right|>|M|$, the subgraph $H$ contains more edges of $M^{\prime \prime}$ than of $M$, and therefore some path-component $P$ of $H$ must start and end with edges of $M^{\prime \prime}$. The origin and terminus of $P$, being covered by $M^{\prime \prime}$, are not covered by $M$. The path $P$ is thus an $M$-augmenting path in $G$, a contradiction.

Hall's Theorem can be restated in a more general version. We denote the matching number, the size of a maximum matching, by $\mu(G)$.

Theorem 1.2.5 Let $G=(X, Y)$ be a bipartite graph, $M$ a matching in $G$, and $U$ the set of $M$-unsaturated vertices in $X$. Then

(a) for any subset $S$ of $X,|U| \geq|N(S)|-|S|$; and

(b) $|U|=|N(S)|-|S|$ if and only if $M$ is a maximum matching of $G$.

Furthermore, the matching number of $G$ is given by

$$
\mu(G)=|X|-\max \{|S|-|N(S)| \mid S \subseteq X\} .
$$

The expression for $\mu(G)$ is known as the König-Ore Formula.

Next, we investigate the properties of matching transformation in bipartite graphs. That is, given two matchings, we can start with one and transform gradually to another one through the operation of symmetric difference.

Theorem 1.2.6 A maximum matching $M$ of a graph $G$ can be obtained from any other maximum matching $M^{\prime}$ by a sequence of transfers along alternating cycles and paths of even lengths. 
Proof. From the proof of Theorem 1.2.4, every component of $G\left[M^{\prime} \triangle M\right]$ is either an alternating cycle or an alternating even path relative to $M^{\prime}$. Changing $M^{\prime}$ in each component in turn will transform $M^{\prime}$ into $M$.

Theorem 1.2.7 (Dulmage and Mendelsohn [438]) Let $G=(X, Y)$ be a bipartite graph and $M_{1}, M_{2}$ be two matchings in $G$. Then there is a matching $M \subseteq M_{1} \cup M_{2}$ such that $M$ covers all the vertices of $X$ saturated by $M_{1}$ and all the vertices of $Y$ saturated by $M_{2}$, that is, $V(M) \supseteq\left(V\left(M_{1}\right) \cap X\right) \cup\left(V\left(M_{2}\right) \cap Y\right)$.

Proof. Let $X_{i}$ (respectively, $Y_{i}$ ) be the vertices of $X$ (of $Y$ ) covered by $M_{i}(i=1,2$ ). Let $G_{1}, G_{2}, \ldots, G_{k}$ be the connected components of $G\left[M_{1} \triangle M_{2}\right]$. Then each $G_{i}$ is an even cycle or path. Let $M_{1 i}=E\left(G_{i}\right) \cap M_{1}$ and $M_{2 i}=E\left(G_{i}\right) \cap M_{2}(1 \leq i \leq k)$ and define a matching $P_{i}$ in each $G_{i}$ :

$$
P_{i}= \begin{cases}M_{1 i} & \text { if } G_{i} \text { is a cycle; } \\ M_{1 i} & \text { if there is a vertex } v \in V\left(G_{i}\right) \cap\left(X_{1}-X_{2}\right) ; \\ M_{2 i} & \text { if there is a vertex } v \in V\left(G_{i}\right) \cap\left(Y_{2}-Y_{1}\right) .\end{cases}
$$

It is not hard to check that $M=\left(M_{1} \cap M_{2}\right) \cup P_{1} \cup P_{2} \cup \cdots \cup P_{k}$ is the required matching.

The local neighborhood condition can be used as a sufficient condition for the maximum matching in bipartite graphs. The following theorem is due to Alon.

Theorem 1.2.8 Let $G=(X, Y)$ be a bipartite graph. If one of the following conditions holds,

(a) $d(x)$ is odd for all $x \in X$ and any two vertices of $X$ have an even number of common neighbors; or

(b) $d(x) \geq 1$ for all $x \in X$ and $d(x) \geq d(y)$ for all $x y \in E(G)$, where $x \in X$ and $y \in Y$,

then $G$ has a matching saturating $X$.

The next result is of type of degree condition.

Theorem 1.2.9 Let $G=(X, Y)$ be a bipartite graph with $|X|=|Y|=n$. If $\delta(G) \geq$ $\frac{n}{2}$, then $G$ has a 1-factor.

A matching is induced if no two edges in the matching are joined by an edge in $G$. Unlike the usual matching problem, the induced matching is a much more difficulty problem to deal with. The problem of finding a maximum induced matching is NPhard, even for bipartite graphs and for planar graphs of maximum degree 4 (see [111]). The polynomial algorithms for solving the maximum induced matching problem are found only for a few families of special graphs (e.g., weakly chordal graphs, circle 
graphs, chordal graphs, and outerplanar graphs). However, we have a neat formula for the size of maximum induced matching, i.e., induced matching number $i \mu(G)$, in bipartite graphs.

Theorem 1.2.10 (Liu and Zhou [381]) Let $G=(X, Y)$ be a connected bipartite graph. Then the size of a maximum induced matching $M$ of $G$ is

$$
i \mu(G)=\max \left\{|S| \mid S \subseteq X \text { such that } N_{G}(T) \neq N_{G}(S) \text { for all } T \subset S\right\} .
$$

Matchings in bipartite graphs and Hall's Theorem have proved to be a valuable tool both in graph theory and in other areas of mathematics. In fact, Hall's Theorem has several equivalent formulations, including the following one in terms of set systems.

Let $\mathcal{A}=\left(A_{i}: i \in I\right)$ be a finite family of (not necessarily distinct) subsets of a finite set $A$. A system of distinct representatives (SDR) for the family $\mathcal{A}$ is a set $\left\{a_{i} \mid i \in I\right\}$ of distinct elements of $A$ such that $a_{i} \in A_{i}$ for all $i \in I$. In this language, Hall's Theorem says that $\mathcal{A}$ has a system of distinct representatives if and only if $\left|\cup_{i \in J} A_{i}\right| \geq|J|$ for all subsets $J$ of $I$. To see that this is indeed a reformulation of Hall's Theorem, let $G=(X, Y)$, where $X=I, Y=A$, and $E(G)=\left\{i a \mid a \in A_{i}\right.$, where $i \in I$ and $\left.a \in A\right\}$.

The other formulations of Hall's Theorem can be found in $(0,1)$-matrix theory and transversal matroid.

\subsection{Matchings in non-bipartite graphs}

In this section, we consider the existence of 1-factors in arbitrary graphs. The characterization of 1-factors was given by Tutte in 1947. It is one of most important theorems in graph theory for several reasons: firstly 1-factor as a 1-regular spanning subgraph is a simplest but the most essential structure of any graph; secondly due to its wide applications in other areas of mathematics and in optimization problems (it often serves as subroutine in many optimization algorithms); finally it is one of earliest topics in graph theory which has a relative complete understanding and matured techniques comparing with other topics in graph theory.

A component $C$ of a graph is called odd if $|V(C)|$ is odd; otherwise, it is called even. Recall that $o(H)$ is the number of odd components in a graph $H$. Using a parity argument, it is not hard to see that

$$
o(G-S)+|S| \equiv|V(G)|(\bmod 2) .
$$

If $G$ is of even order, then $|V(G)| \equiv 0(\bmod 2)$ and thus

$$
o(G-S) \equiv|S|(\bmod 2) .
$$


This fact is quite handy when estimating the number of odd components in a graph.

Now we present Tutte's criterion for 1-factors. Besides Tutte's original proof for this theorem, there are several shorter and simpler proofs, respectively, from Anderson (1971), Lovász (1975), as well as Ore's transformation from bipartite graphs. Here we use the proof from Lovász.

Theorem 1.3.1 (Tutte's 1-Factor Theorem, Tutte (1947)) A graph G has a 1-factor if and only if for any $S \subseteq V(G)$

$$
o(G-S) \leq|S|
$$

Proof. Necessity. Since the odd components of $G-S$ must have vertices matched to distinct vertices of $S$, we have $o(G-S) \leq|S|$.

Sufficiency. Note that adding an edge joining two components of $G-S$ does not increase the number of odd components. Hence (1.4) is preserved from the addition of edges. That is, let $G^{\prime}=G \cup e$ and $S \subseteq V(G)$, then $o\left(G^{\prime}-S\right) \leq o(G-S) \leq|S|$. Furthermore, if $G^{\prime}$ has no 1-factor, then $G$ has no 1-factor either.

Assume that $G$ satisfies (1.4) but has no 1-factor. Without loss of generality, let $G$ be (edge-wise) maximal such a graph. Note that (1.4) implies that $|V(G)|$ is even (considering $S=\emptyset$ ).

Let $S_{0}$ be the set of vertices in $G$ that have degree $|V(G)|-1$.

Case 1. $G-S_{0}$ consists of disjoint complete graphs.

In this case, vertices in each component of $G-S_{0}$ can be paired in any way, with one extra in the odd components. Since $o\left(G-S_{0}\right) \leq\left|S_{0}\right|$ and each vertex of $S_{0}$ is adjacent to all of $G-S_{0}$, we can match the leftover vertices to vertices of $S_{0}$.

The remaining vertices are in $S_{0}$, which is a clique. Since $V(G)$ is even, by parity, an even number of vertices remain in $S_{0}$ and thus we can form a 1-factor.

Case 2. $G-S_{0}$ is not a disjoint union of cliques.

In this case, $G-S_{0}$ has two vertices at distance 2 ; these are nonadjacent vertices $x, z$ with a common neighbor $y \notin S_{0}$. Furthermore, $G-S_{0}$ has another vertex $w$ not adjacent to $y$, since $y \notin S_{0}$. By the maximality of $G$, adding an edge to $G$ creates a 1-factor; let $M_{1}$ and $M_{2}$ be 1-factors in $G \cup x z$ and $G \cup y w$, respectively. It suffices to show that $M_{1} \triangle M_{2}$ contains a 1-factor avoiding $x z$ and $y w$, because this will be a 1-factor of $G$.

Let $F=M_{1} \triangle M_{2}$. Since $x z \in M_{1}-M_{2}$ and $y w \in M_{2}-M_{1}$, both $x z$ and $y w$ are in $F$. Since every vertex of $G$ has degree 1 in each $M_{1}$ and $M_{2}$, every vertex of $G$ has degree 0 or 2 in $F$. Hence the components of $F$ are even cycles and isolated vertices.

Let $C$ be the cycle of $F$ containing $x z$ (see Figure 1.3). If $C$ does not also contain $y w$, then the desired 1-factor consists of the edges of $M_{2}$ from $C$ and all of $M_{1}$ not 


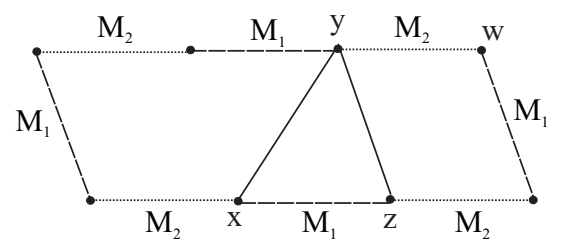

Figure 1.5: Alternative Paths

in $C$. If $C$ contains both $y w$ and $x z$, then to avoid them we use $y x$ or $y z$. In the portion of $C$ starting from $y$ along $y w$, we use edges of $M_{1}$ to avoid using $y w$. When we reach $\{x, z\}$, we use $z y$ if we arrive at $z$; otherwise, we use $x y$. In the remainder of $C$ we use the edges of $M_{2}$. We have produced a 1-factor of $C$ that does not use $x z$ or $y w$. Combined with $M_{1}$ or $M_{2}$ outside $C$, we have a 1 -factor of $G$.

Hall's Theorem and Tutte's 1-Factor Theorem are in fact equivalent. They both belong to a large family of fundamental results in graph theory which are all equivalent to each other (see Jacobs and Heiratssatz [266]).

As one of applications of 1-Factor Theorem and also a refinement, Theorem 1.3.1 can also be restated in terms of factor-critical graphs. This result was a by-product of the algorithm for finding a maximum cardinality matching by Edmonds [168]. A non-algorithmic proof of this theorem was given by Anderson [55].

Let $f c(G)$ be the number of factor-critical components of graph $G$.

Theorem 1.3.2 (Edmonds [168]) A graph $G$ of even order has a 1-factor if and only if for every subset $S \subseteq V(G)$,

$$
f c(G-S) \leq|S|
$$

Proof. Since every factor-critical component is an odd component, so the necessity follows immediately from 1-Factor Theorem.

Conversely, to the contrary, assume that $G$ has no 1-factor. Then, by 1-Factor Theorem, there exists a subset $S \subseteq V(G)$ such that $o(G-S)>|S|$ and $S \neq \emptyset$ or $V(G)$. Note the fact

$$
o\left(G-S^{\prime}\right) \leq\left|S^{\prime}\right| \text { for all } S_{0} \subset S^{\prime} \subseteq V(G) .
$$

Choose maximal such a set $S_{0}$. Then the maximality of $S_{0}$ implies that $G-S_{0}$ has no even component. Next we show that every component of $G-S_{0}$ is factor-critical, i.e., $f c\left(G-S_{0}\right)=o\left(G-S_{0}\right)$.

Let $C$ be an odd component of $G-S_{0}$, and $v$ any vertex of $C$. Then for every $T \subseteq V(C-v),(1.5)$ implies 


$$
\begin{aligned}
\left|S_{0}\right|+1+|T| & \geq o\left(G-\left(S_{0} \cup\{v\} \cup T\right)\right) \\
& =o\left(G-S_{0}\right)-1+o(C-(\{v\} \cup T)) \\
& >\left|S_{0}\right|-1+o((C-v)-T) .
\end{aligned}
$$

Hence $o((C-v)-T)<|T|+2$, which implies $o((C-v)-T) \leq|T|$ by (1.2). Therefore $C-v$ has a 1 -factor by 1 -Factor Theorem, or $C$ is factor-critical. Consequently, $f c\left(G-S_{0}\right)=o\left(G-S_{0}\right)>\left|S_{0}\right|$, a contradiction to the assumption of the theorem.

In fact, Tutte's 1-Factor Theorem can be refined further by considering only the special subsets $S$ (i.e., barrier set) and the number of factor-critical components. More refinement, i.e., Gallai-Edmonds Structure Theorem, will be discussed in Section 1.4.

When applying 1-Factor Theorem to various classes of graphs, the criterion can be much simpler and nicer. The next result is such an example and it can be proven easily by induction.

Proposition 1.3.1 (Chungphaisan (1976)) A tree $T$ of even order has a 1-factor if and only if $o(T-v)=1$ for every vertex $v$ of $T$.

Augmenting path (or chain) technique lies in the heart of factor theory. It is an effective way to adjust a subgraph to get a better subgraph (or factor). Its significance is also reflected in the fact that if an augmenting technique is developed for a given factor problem (e.g., 1 -factors, $k$-factors, $f$-factors, $L$-factors with $(*)$ property, etc.), then it often can be converted into a polynomial algorithm for the problem.

Augmenting path (or chain) technique posses many nice properties, as shown in the next theorem. These properties are important for the adjustment process to reach the optimal subgraphs. The uttermost usage of such properties can be found in treating $L$-factors in [406, 407].

For a matching $M$ and a vertex set $S$, if $V(M) \cap S=\emptyset$, then we say that $M$ avoids $S$.

Theorem 1.3.3 (see [13]) Let $A$ and $B$ be vertex subsets of a graph $G$ such that $|A|<|B|$. Then

(a) if there exist two matchings, one saturating $A$ and the other saturating $B$, then there exists a matching that saturates $A$ and at least one vertex in $B-A$;

(b) if there exist two maximum matchings, one avoiding $A$ and the other avoiding $B$, then there exists a maximum matching that avoids $A$ and at least one vertex in $B-A$. 
Proof. (a) Let $M_{A}$ and $M_{B}$ be matchings in $G$ which saturate $A$ and $B$, respectively. If $M_{A}$ saturates one vertex in $B-A$, then $M_{A}$ itself is the desired matching. Hence we may assume that $M_{A}$ avoids $B-A$.

Consider the subgraph $G\left[M_{A} \triangle M_{B}\right]$. Then for every vertex $b$ in $B-A$, there exists a path in $G\left[M_{A} \triangle M_{B}\right]$ starting with $b$, which may end with a vertex in $A-B$. Since $|A|<|B|$ and no path of $G\left[M_{A} \triangle M_{B}\right]$ ends with a vertex in $A \cap B$, there exists a path $P$ in $G\left[M_{A} \triangle M_{B}\right]$ that starts with $b_{1} \in B-A$ and ends with $x \notin A$. Since $P$ is an $M_{A}$-alternating path connecting $b_{1}$ and $x, M_{A} \triangle E(P)$ is the desired matching in $G$, which saturates $A$ and $b_{1} \in B-A$.

(b) Let $N_{A}$ and $N_{B}$ be maximum matchings in $G$ which avoid $A$ and $B$, respectively. We may assume that $N_{A}$ saturates $B-A$ since otherwise $N_{A}$ itself is the desired matching. Then by the same argument as in (a), there exists a $N_{A}$-alternating path $P$ in $G\left[N_{A} \triangle N_{B}\right]$ starting with a vertex $b \in B-A$ and ending with a vertex not contained in $A$. If both end-edges of $P$ are contained in $N_{A}$, then $N_{B} \triangle E(P)$ is a matching with $\left\|N_{B}\right\|+1$ edges, contrary to the maximality of $N_{B}$. Thus one endedge of $P$ belongs to $N_{B}$, and thus $N_{A} \triangle E(P)$ is the desired maximum matching in $G$, which avoids $A$ and $b \in B-A$.

To find a matching containing a given set of vertices, we have the following interesting result.

Proposition 1.3.2 (Kierstead [316]) Let $G$ be a graph and $T$ the set of vertices of degree $\Delta(G)$. If $G[T]$ is a bipartite subgraph, then there exists a matching in $G$ saturating all vertices of $T$.

To conclude this section, we present a conjecture due to Macajova and Skoviera.

Conjecture 1.3.1 (Macajova and Skoviera (2005)) Every 2-edge-connected cubic graph has two perfect matchings $M_{1}$ and $M_{2}$ such that $M_{1} \cap M_{2}$ does not contain an odd edge-cut.

\subsection{Sufficient conditions for 1-factors}

Although Tutte's 1-Factor Theorem provides a nice and simple criterion for 1-factors, to check the condition (1.4) is a non-trivial task. So many researchers have attempted to find simple sufficient conditions for the existence of 1-factors by using various graphic parameters, or to determine special classes of graphs to be 1-factorable. The graphic parameters having been studied include connectivity, claw-free, neighborhood, degree-sequence, toughness, binding number, etc. Those approaches not only provide a simple conditions for existence of 1-factors, and also enrich our understanding to the structures of graphs with 1-factors; more importantly it supplies many fresh ideas for the study of other factors. In fact, many of them have been generalized to $k$-factors, $[a, b]$-factors and so on. We will examine it in more details in later chapters, 
for instance, toughness in Sections 3.1 and 3.2, binding number in Section 3.3 and connectivity in Section 3.4.

When we use 1-Factor Theorem to show the existence of 1-factors or maximum matchings in a graph, often we have to deal with the number of odd components and counting the number of edges between odd components and a vertex set. Beside the relation (1.2), the following result will be handy for such estimations, in particular, in regular graphs.

Proposition 1.4.1 Let $G$ be a r-regular graph $(r \geq 2)$, and $S \subseteq V(G)$. Then for each odd component $C$ of $G-S$,

$$
e_{G}(C, S) \equiv r \quad(\bmod 2)
$$

In particular, if $G$ is an $(r-1)$-edge-connected $r$-regular graph, then $e_{G}(C, S) \geq r$.

Proof. Since $|C|$ is odd, we have

$$
r \equiv r|C|=\sum_{u \in V(C)} d_{G}(u)=e_{G}(C, S)+2|E(C)| \equiv e_{G}(C, S) \quad(\bmod 2) .
$$

Moreover, if $G$ is an $(r-1)$-edge-connected $r$-regular graph, then $e_{G}(C, S) \geq r-1$, and by combining this inequality and parity, we have $e_{G}(C, S) \geq r$.

Next, we examine different parameter as sufficient condition for 1-factors.

Connectivity Condition:

Let us start with connectivity, which usually the first parameter one looks at, as a condition for the existence of 1-factors. The first significant result in this direction, actually the first on 1-factors in graphs, was obtained by Petersen (1891), who was particularly interested in 3-regular graphs.

Theorem 1.4.1 (Petersen [475]) Let $G$ be a 2-edge-connected 3-regular graph. Then $G$ has a 1-factor.

Proof. Let $S$ be any nonempty proper subset of $V(G)$. Denote all the odd components of $G-S$ by $C_{1}, C_{2}, \ldots, C_{m}$. From Proposition 1.4.1, we have $e_{G}\left(C_{i}, S\right) \geq 3$ for $1 \leq i \leq m$. Therefore

$$
3|S| \geq e_{G}\left(C_{1} \cup C_{2} \cup \cdots \cup C_{m}, S\right) \geq 3 m
$$

Hence $|S| \leq m=o(G-S)$ or $G$ has a 1-factor by 1-Factor Theorem. 


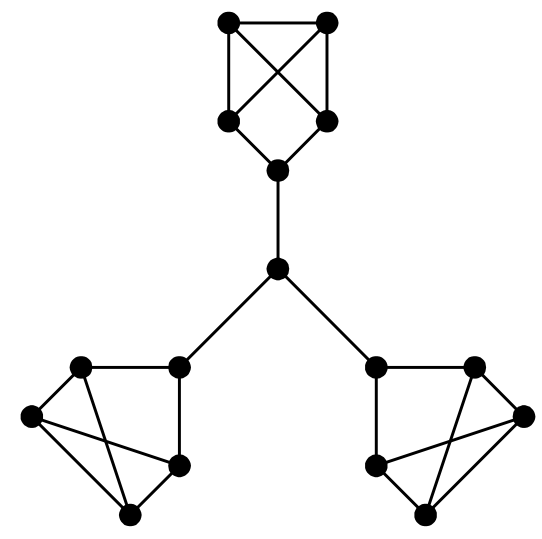

Figure 1.6: A cubic graph without 1-factors

The connectivity condition in Theorem 1.4.1 is best possible, i.e., there exist connected cubic graphs which have no 1-factor. An example is shown in Figure 1.6.

This theorem can be generalized in several ways. Firstly, the 2-edge-connectivity condition can be replaced by "all the bridges of $G$ are contained in the same path"; secondly, the theorem also holds for multigraphs as well; finally, of course, we can generalize it to general $r$-regular graphs. In fact, we prove a slightly stronger result by specifying a 1-factor to pass a given edge or to avoid other edges.

Theorem 1.4.2 Let $r \geq 2$, and $G$ be an $(r-1)$-edge-connected $r$-regular graph.

(a) If $|V(G)| \equiv 0$ (mod 2), then, for every edge e of $G, G$ has a 1-factor containing e. In particular, $G$ has a 1-factor;

(b) if $|V(G)| \equiv 1$ (mod 2), then, for every vertex $v, G-v$ has a 1-factor. That is, $G$ is factor-critical.

Proof. (a) Suppose, to the contrary, that there exists an edge $e=x y$ such that no 1-factor containing $e$. Then $G-\{x, y\}$ has no 1-factor. From 1-Factor Theorem, there exists $S^{\prime} \subseteq V(G)-\{x, y\}$ such that $o\left(G-\{x, y\}-S^{\prime}\right)>\left|S^{\prime}\right|$. Let $S=S^{\prime} \cup\{x, y\}$. Then $o(G-S) \geq\left|S^{\prime}\right|+2=|S|$ by parity. Let $C_{1}, C_{2}, \ldots, C_{m}$ be the odd components of $G-S$, where $m=o(G-S)$. Then, by Proposition 1.4.1, we have $e_{G}\left(C_{i}, S\right) \geq r$. Hence

$$
|\nabla(S)| \leq r|S|-2 \leq r m-2 \text { since } e=x y \text { is in } G[S]
$$

and

$$
|\nabla(S)| \geq e_{G}\left(C_{1} \cup C_{2} \cup \cdots \cup C_{m}, S\right) \geq r m,
$$

a contradiction. Hence, for every edge $e$ of $G, G$ has a 1-factor containing $e$. 
(b) Suppose, to the contrary, that $G$ is not factor-critical. Then, by Theorem ?? (in Section 6.1), there exists $\emptyset \neq S \subseteq V(G)$ such that $o(G-S)>|S|-1$ or $o(G-S) \geq|S|+1$ by parity. Let $C_{1}, C_{2}, \ldots, C_{m}$ be the odd components of $G-S$, where $m=o(G-S)$. Then, by Proposition 1.4.1, we have $e_{G}\left(C_{i}, S\right) \geq r$. Hence

$$
r|S| \geq|\nabla(S)| \geq e_{G}\left(C_{1} \cup C_{2} \cup \cdots \cup C_{m}, S\right) \geq r m \geq r(|S|+1)
$$

a contradiction. Hence $G$ is factor-critical.

The edge-connectivity condition in Theorem 1.4.2 is best possible, i.e., there exist infinitely many $(r-2)$-edge-connected $r$-regular graphs of even order that have no 1-factor. The above theorem holds for multigraphs as well. Note that in part (b) $r$ must be an even integer.

A classic result due to Plesník (1974) showed that there exist 1-factors to avoid a set of edges in regular graphs with edge-connectivity condition. The proof is from [13].

Theorem 1.4.3 (Plesník [479]) Let $r \geq 2$ be an even integer, and $G$ be an $(r-1)$ edge-connected $r$-regular graph of even order. Then for any $r-1$ edges $e_{1}, e_{2}, \ldots, e_{r-1}$ of $G, G$ has a 1-factor excluding $\left\{e_{1}, e_{2}, \ldots, e_{r-1}\right\}$.

Proof. Let $H=G-\left\{e_{1}, \ldots, e_{r-1}\right\}$. We show that $H$ has a 1 -factor. For any vertex set $S \subseteq V(H)=V(G)$, let $C_{1}, C_{2}, \ldots, C_{m}$ be the odd components of $H-S$, where $m=o(H-S)$. Then by the same argument as in the proof of Proposition 1.4.1, we have

$$
e_{G}\left(C_{i}, V(G)-V\left(C_{i}\right)\right) \geq r-1 \text { and } e_{G}\left(C_{i}, V(G)-V\left(C_{i}\right)\right) \equiv r(\bmod 2) .
$$

Hence

$$
r \leq e_{G}\left(C_{i}, V(G)-V\left(C_{i}\right)\right)=e_{G}\left(C_{i}, S\right)+e_{G}\left(C_{i}, V(G)-S-V\left(C_{i}\right)\right) .
$$

Since $C_{i}$ and $C_{j}(i \neq j)$ are components of $H-S$, if there is an edge $e$ between $C_{i}$ and $C_{j}$, then $e \in\left\{e_{1}, \ldots, e_{r-1}\right\}$. That is, $E_{G}\left(C_{i}, V(G)-S-V\left(C_{i}\right)\right) \subseteq\left\{e_{1}, \ldots, e_{r-1}\right\}$ and each of $\left\{e_{1}, \ldots, e_{r-1}\right\}$ appears in at most two such sets $E_{G}\left(C_{i}, V(G)-S-V\left(C_{i}\right)\right)$, and so it yields

$$
\sum_{i=1}^{m} e_{G}\left(C_{i}, V(G)-S-V\left(C_{i}\right)\right) \leq 2\left|\left\{e_{1}, e_{2}, \ldots, e_{r-1}\right\}\right|=2(r-1) .
$$

Therefore

$$
\begin{aligned}
r m & \leq \sum_{i=1}^{m}\left(e_{G}\left(C_{i}, S\right)+e_{G}\left(C_{i}, V(G)-S-V\left(C_{i}\right)\right)\right) \\
& \leq \sum_{x \in S} d_{G}(x)+\sum_{i=1}^{m} e_{G}\left(C_{i}, V(G)-S-V\left(C_{i}\right)\right) \\
& \leq r|S|+2(r-1) .
\end{aligned}
$$


Hence $m \leq|S|+2(r-1) / r<|S|+2$, which implies $m \leq|S|$ by parity. That is, $o(H-S) \leq|S|$ or $H$ has a 1 -factor.

Note that from Theorem 1.4.3, we can give a short proof to Theorem 1.4.2 (a): for a given edge $e=x y$, let the set of other $r-1$ edges incident to the vertex $x$ be $X$, then there exists a 1-factor $F$ excluding $X$ and thus $F$ is a 1-factor containing $e$.

Also note that the above result holds for multigraphs as well.

This result is best possible in the sense that there exist infinitely many $(r-2)$ edge-connected $r$-regular graphs of even order that have no 1-factor.

\section{Claw-free Condition:}

A graph containing no $K_{1,3}$ as an induced subgraph is said to be claw-free. Clawfree graphs have been a popular condition to study for many graphic parameters. An important reason is that under claw-free condition many graph theory problem will become significantly simpler. For instance, to determine a maximum independent set in arbitrary graphs is a $N P$-hard problem but becomes a $P$-problem if restricting to claw-free graphs.

Now we consider 1-factors in claw-free graphs.

Theorem 1.4.4 (Sumner [539])

(a) If $G$ is a connected claw-free graph of even order, then has a 1-factor;

(b) If a $k$-connected graph $(k \geq 2)$ of even order has no induced subgraph isomorphic to $K_{1, k+1}$, then $G$ has a 1 -factor.

Proof. (a) Use induction on $|G|$. Let $P=v_{1}, v_{2}, \ldots, v_{k}$ be a longest path of $G$. Then $k \geq 3$. It follows $N_{G}\left(v_{1}\right) \subseteq V(P)-\left\{v_{1}\right\}$.

Claim. $G-\left\{v_{1}, v_{2}\right\}$ is connected.

If $d_{G}\left(v_{2}\right)=2$, then $G-\left\{v_{1}, v_{2}\right\}$ is connected since $N_{G}\left(v_{1}\right) \subseteq V(P)-\left\{v_{1}\right\}$. If $d_{G}\left(v_{2}\right) \geq 3$. For every $x \in N_{G}\left(v_{2}\right)-V(P), x v_{3}$ must be an edge of $G$ since, otherwise $v_{1} x$ or $v_{1} v_{3}$ is an edge of $G$ as the vertices $x, v_{1}, v_{2}, v_{3}$ do not induce a claw. However, this implies that $G$ contains a path longer than $P$, a contradiction.

Therefore $G-\left\{v_{1}, v_{2}\right\}$ is connected, and of course, is claw-free, and has even order. By the induction hypothesis, $G-\left\{v_{1}, v_{2}\right\}$ has a 1 -factor and so does $G$.

(b) Suppose, to the contrary, that $G$ has no 1-factor. By 1-Factor Theorem, then there exists a subset $S \subseteq V(G)$ such that $o(G-S) \geq|S|+2$ by parity. Assume that $S$ is a minimal such set and let $|S|=m$. Denote the odd components of $G-S$ by $O_{1}, O_{2}, \ldots, O_{t}$. Thus $t \geq m+2$. Moreover, $\mid\left\{v \in S \mid v\right.$ is adjacent to a vertex of $\left.O_{i}\right\} \mid \geq$ $k$, for each $i=1,2, \ldots, t$, and so there are at least $k$ edges from $O_{i}$ to $S$ having distinct end-vertices in $S$. Choosing such a collection of $k$ edges for each $i$ gives a collection 
of $k t \geq k(m+2)$ edges and hence some vertex $x \in S$ is incident with at least $k+1$ of these edges. But then $x$ is the center of an induced $K_{1, k+1}$, a contradiction.

The above theorem can be generalized in several ways. Here we mention one by Ryjáček [509] and others are due to Nebeský [445]. We say that a graph $G$ is almost claw-free if the centers of induced claws in $G$ are independent and their neighborhoods are 2-dominated. It can be showed that every almost claw-free graph is $K_{1,5}$-free.

Theorem 1.4.5 (Ryjáček [509]) Every connected almost claw-free graph of even order has a 1-factor.

Given a graph $G$, if it does not have 1-factors, by 1-Factor Theorem, there exists a vertex set $S_{0}$ such that $o\left(G-S_{0}\right)>\left|S_{0}\right|$ and $o\left(G-S_{0}\right)-\left|S_{0}\right|=\max _{S \subseteq V(G)} o(G-S)-|S|$ (Note that this set is called a barrier set). Sumner [538] showed that there exists a barrier set $S_{0}$, each vertex of $S_{0}$ is a claw center. In fact, a minimal barrier set is such a set. With this remark, Tutte's 1-Factor Theorem can be restated in the following form.

Theorem 1.4.6 (Sumner [538]) A graph $G$ has 1-factor if and only if for any set $S$ consisting of claw centers,

$$
o(G-S) \leq|S|
$$

Neighborhood Condition:

Next we discuss the existence of 1-factors in terms of neighborhood condition.

Theorem 1.4.7 (Win [591]) Let $G$ be a graph of even order $n$. If for any two nonadjacent vertices $x$ and $y$,

$$
d_{G}(x)+d_{G}(y) \geq n+1
$$

then $G$ has a hamiltonian cycle disjoint from a 1-factor. In particular, G has three disjoint 1-factors.

Theorem 1.4.8 (Anderson [55]) Let $G$ be a graph of even order $n$. If, for any subset $S \subseteq V(G)$ with $|S| \leq \frac{3 n}{4}$, we have $\left|N_{G}(S)\right| \geq \frac{4}{3}|S|$, then $G$ has a 1-factor.

Proof. Suppose, to the contrary, that $G$ has no 1-factor. By 1-Factor Theorem, then there exists a vertex-subset $S_{0} \subseteq V(G)$ such that $o\left(G-S_{0}\right)>\left|S_{0}\right|$.

Case 1. $\left|S_{0}\right| \geq \frac{n}{4}$.

Set $S=V(G)-S_{0}$. Then $|S| \leq \frac{3}{4} n$ and thus $\left|N_{G}(S)\right| \geq \frac{4}{3}|S|$. That is,

$$
\frac{4}{3}\left(n-\left|S_{0}\right|\right) \leq\left|N_{G}\left(G-S_{0}\right)\right| \leq n-i\left(G-S_{0}\right)
$$


i.e.,

$$
i\left(G-S_{0}\right) \leq \frac{4}{3}\left|S_{0}\right|-\frac{n}{3} .
$$

Thus

$$
\begin{aligned}
n & \geq\left|S_{0}\right|+i\left(G-S_{0}\right)+3\left(o\left(G-S_{0}\right)-i\left(G-S_{0}\right)\right) \\
& >\left|S_{0}\right|+3\left|S_{0}\right|-\frac{8}{3}\left|S_{0}\right|+\frac{2}{3} n .
\end{aligned}
$$

Hence $\left|S_{0}\right|<\frac{n}{4}$, a contradiction.

Case 2. $\left|S_{0}\right|<\frac{1}{4} n$.

Set $S=V(G)-S_{0}$. Then $|S|>\frac{3}{4} n$. Let $S^{\prime}$ be any subset of $S$ with $\left|S^{\prime}\right|=\frac{3}{4} n$. Then $\left|N_{G}\left(S^{\prime}\right)\right| \geq \frac{4}{3}\left|S^{\prime}\right|=n$ and so $V(G) \subseteq N_{G}(S)$. Thus the vertices of $S$ are adjacent to all the vertices of $G$. Consequently, there are no singleton components in $G-S_{0}$ and thus there are at least $\left|S_{0}\right|+2$ odd components with at least 3 vertices in $G-S_{0}$. Consider all but one of these components. There are, say, $h$ vertices in their union, where $h \geq 3\left|S_{0}\right|+3$. But they can be adjacent to at most $h+\left|S_{0}\right| \leq h+\frac{1}{3}(h-3)<\frac{4}{3} h$ vertices. This contradiction completes the proof.

Note that the value $\frac{4}{3}$ in the above theorem is best possible. To see this, consider the graph $G=(r+2) K_{3} \vee\left(r K_{1}\right)$. Let $S=V\left(r K_{1}\right)$. Then $o(G-S)=r+2>|S|=r$ and thus $G$ has no 1-factor. Let $S^{\prime}=V\left((r+1) K_{3}\right)$. The $\left|N_{G}\left(S^{\prime}\right)\right|=3(r+1)+r$. Since $(4 r+3) /(3 r+3)$ can be made as close to $\frac{4}{3}$ as we like, it follows that the value $\frac{4}{3}$ is best possible.

Degree Sequences:

Let $F$ be a 1-factor of a graph $G$. Then $G-F$ is a subgraph with the degree one less than that in $G$ for each vertex, i.e., $d_{G-F}(x)=d_{G}(x)-1$ for any $x \in V(G)$. This obvious fact turns out to be also sufficient for the existence of 1-factors in a graph. The next theorem gives a condition for the existence of 1-factors in terms of realization of degree sequences.

Theorem 1.4.9 (Kundu [332]; Lovász [410]) Let $G$ be a graph of even order with degree sequence $d_{1}, d_{2}, \ldots, d_{n}$. If $d_{1}-1, d_{2}-1, \ldots, d_{n}-1$ is a degree sequence of some graph, then $G$ has a 1-factor.

Proof. Let $d_{1}, d_{2}, \ldots, d_{n}$ be the degree sequence of $G$ and $d_{1}-1, d_{2}-1, \ldots, d_{n}-1$ the degree sequence of another graph $G^{\prime}$ with $V=V(G)=V\left(G^{\prime}\right)$. Choose $G$ and $G^{\prime}$ so that the symmetric difference of $E(G)$ and $E\left(G^{\prime}\right), E(G) \triangle E\left(G^{\prime}\right)$, is minimal. To show that $G$ has a 1 -factor, we need only to show that $G^{\prime} \subseteq G$.

Suppose that $G^{\prime} \nsubseteq G$, i.e., $E\left(G^{\prime}\right) \nsubseteq E(G)$. Let $x_{0}$ be a vertex which is incident with the most number, say $r$, of edges in $E\left(G^{\prime}\right)-E(G)$. Since $d_{G}\left(x_{0}\right)=d_{G^{\prime}}\left(x_{0}\right)+1$, the definition of symmetric difference implies that there are $r+1$ edges in $E(G)-E\left(G^{\prime}\right)$ 
incident with $x_{0}$. Due to the choice of $x_{0}$ and $E\left(G^{\prime}\right) \nsubseteq E(G)$, there exists a vertex $z \in V$ such that $z x_{0} \in E\left(G^{\prime}\right)-E(G)$ and then we pick up another vertex $w \in V$ with $z w \in E(G)-E\left(G^{\prime}\right)$. Note that such a $w$ exists since $d_{G}(z)=d_{G^{\prime}}(z)+1$.

Claim 1. For any $y \in V-\left\{x_{0}, w\right\}$ with $x_{0} y \in E(G)-E\left(G^{\prime}\right)$, then $w y \in E(G)-$ $E\left(G^{\prime}\right)$.

Otherwise, then $w y \notin E(G)$ or $w y \in E\left(G^{\prime}\right)$. If $w y \notin E(G)$, then we replace the edges $x_{0} y$ and $w z$ by the edges $x_{0} z$ and $w y$ in $G$. The resulting graph has the same degree sequence, but with smaller symmetric difference with respect to $G^{\prime}$, a contradiction to the minimality. Similarly, if $w y \in E\left(G^{\prime}\right)$, then replacing the edges $x_{0} z$ and $w y$ by the edges $x_{0} y$ and $w z$ in $G^{\prime}$ yields a contradiction, again.

From Claim 1, for every edge $y x_{0} \in E(G)-E\left(G^{\prime}\right)$, we have the edge $y w \in$ $E(G)-E\left(G^{\prime}\right)\left(y \neq x_{0}, w\right)$. In addition, there is also the edge $w z$. Note that the edge $x_{0} w$ may or may not be an edge of $E(G)-E\left(G^{\prime}\right)$, but it contributes the same number to $d_{G}\left(x_{0}\right)$ and $d_{G}(w)$ in any case. Thus, we see that the number of edges in $E(G)-E\left(G^{\prime}\right)$ incident with $w$ is greater than the number of edges in $E(G)-E\left(G^{\prime}\right)$ incident with $x_{0}$, a contradiction to the choice of $x_{0}$.

The above theorem can also be rewritten as a sufficient and necessary condition for the realization of degree sequence in graphs with 1-factors.

Corollary 1.4.1 Let $d_{1}, d_{2}, \ldots, d_{n}$ be integers. Then $d_{1}, d_{2}, \ldots, d_{n}$ is a degree sequence of a graph with a 1-factor if and only if $n$ is even and both sequences $d_{1}, d_{2}, \ldots, d_{n}$ and $d_{1}-1, d_{2}-1, \ldots, d_{n}-1$ are degree sequences of graphs.

Induced Subgraph and 1-Factors:

The existence of 1-factors in subgraphs can also ensure the existence of 1-factors in the original graph. This fact is firstly proved by Sumner [539]. In fact, this idea turns out to be very fruitful and can be applied to study many properties related to matchings. We shall present several results about extendability in induced subgraphs in Section 6.2 later.

Theorem 1.4.10 (Sumner [539]) If $G$ is a connected graph of order $2 n(n \geq 2)$ and $k$ is an integer, $1 \leq k \leq n$, such that every connected induced subgraph of order $2 k$ has a 1-factor, then $G$ has a 1-factor.

Proof. We use induction on $n$. It is easy to check the validity of the theorem for $n=2$, 3. Suppose that it holds for some $n-1(n \geq 4)$. Let $G$ and $k$ be given in the theorem. Clearly, we may assume that $k \leq n-1$, since the case of $k=n$ is trivial.

It is easy to see that $G$ does not contain two leaves adjacent to a same vertex $z$, otherwise these two leaves together with $z$ can be extended to a connected induced 
subgraph of order $2 k$ which has no 1 -factor.

Claim 1. There exist two adjacent vertices $x, y \in V(G)$ such that $G-\{x, y\}$ is connected.

Without loss of generality, assume that $G$ is not complete and thus $G$ has diameter $d \geq 2$. Let $a, y \in V(G)$ be vertices of $G$ which are a distance $d$ apart and let $P=a \ldots x y$ be a path of length $d$ joining $a$ and $y$. Suppose that $G^{\prime}=G-\{x, y\}$ is not a connected graph. Let $C$ be the component of $G^{\prime}$ containing $a$. Since $P$ is a diameter of $G$, every vertex in $G^{\prime}-C$ is adjacent to $x$. Thus, if $G^{\prime}-C$ contains a nontrivial component $B$, then for any $b, c \in B$ which are adjacent, every vertex in $G-\{b, c\}$ is joined to $x$ by a path, and hence $G-\{b, c\}$ is connected. Similarly, if there exists a vertex $e \in G^{\prime}-C$ which is adjacent to $y$, then $G-\{e, y\}$ is connected. Hence we may suppose that $G^{\prime}-C$ contains only isolated vertices and each of these vertices is adjacent only to $x$. Thus, every vertex in $G^{\prime}-C$ is a leaf of $G$ adjacent to $x$. Therefore, since $G$ has no two leaves adjacent to a same vertex, $G^{\prime}-C$ must consist of a single isolated vertex $f$. If $y$ were a leaf of $G$, then $f$ and $y$ would be leaves adjacent to a same vertex. Hence, we may assume that $y$ is adjacent to some vertex of $C$, and so $G-\{x, y\}$ is connected.

From Claim 1, $G-\{x, y\}$ is connected and inductive assumption implies that $G-\{x, y\}$ has a 1 -factor $F$. Hence, $F \cup x y$ constitutes a 1-factor of $G$.

Theorem 1.4.11 (Sumner [539]) If $G$ is a connected graph of even order and every induced $K_{1,3}$ contains a bridge whose deletion results in two components of even order, then $G$ has a 1-factor.

Proof. Use induction on $|G|$. It is easy to check the theorem holds for small orders. Assume the theorem holds for graphs with fewer vertices than $|G|$.

If $G$ has no induced $K_{1,3}$, then $G$ has a 1-factor by Theorem 1.4.4 (a). If $G$ does contain an induced $K_{1,3}$, let $e=a b$ be an edge of this $K_{1,3}$ such that the deletion of $e$ results in a graph having two even components $A$ and $B$ with $a \in A$ and $b \in B$. Suppose $A$ contains an induced $K_{1,3}$. Let $f$ be an edge of this subgraph such that $G-f$ contains two components of even order. Since both end-vertices of $f$ lie in $A$, $A-f$ consists of two components $C$ and $D$. We may assume that $a \in D$. Then $C$ is one of the components of $G-f$ and hence has even order (as must $D$ also). Thus $A$ satisfies the condition of the theorem and so must have a 1-factor by induction hypothesis. Similarly, $B$ contains a 1 -factor which together with that in $A$ produces a 1 -factor in $G$.

\section{Other Conditions:}

Many other parameters, e.g., toughness, binding number, independent number, genus, etc., can be used as sufficient conditions for 1-factors. Below we include two such theorems as conclusion of this section. 
Let $G$ be a connected graph. The toughness of $G$ was introduced by Chvátal [153] and defined as

$$
t(G)=\min \left\{\frac{|S|}{\omega(G-S)} \mid S \subseteq V(G), \omega(G-S) \geq 2\right\},
$$

if $G$ is not a complete graph; $t(G)=\infty$ if $G$ is a complete graph. If $t(G) \geq k$, then we call $G k$-tough.

Theorem 1.4.12 (Chvátal [153]) Let $G$ be a connected 1-tough graph of even order. Then $G$ has a 1-factor.

Proof. For any nonempty subset $S \subset V(G)$, if $\omega(G-S) \geq 2$, then

$$
|S| \geq \frac{|S|}{t(G)} \geq \omega(G-S) \geq o(G-S),
$$

or $o(G-S) \leq|S|$. If $\omega(G-S)=1$, then $o(G-S) \leq \omega(G-S)=1 \leq|S|$ since $S$ is nonempty. When $S=\emptyset$, then $o(G-S)=o(G)=0=|S|$ as $G$ is of even order. That is, $o(G-S) \leq|S|$ for any subset $S$ and thus $G$ has a 1 -factor.

The last parameter to consider is genus. The genus, $\gamma^{*}(G)$, of a graph $G$ is defined to be smallest genus (i.e., number of handles) of all surfaces on which $G$ can be embedded.

Theorem 1.4.13 (Nishizeki [466]) Let $G$ be a $n$-connected graph of even order. If $\gamma^{*}(G)<n(n-2) / 4$, then $G$ has a 1 -factor.

\subsection{Gallai-Edmonds Structure Theorem}

In this section, we present a structural theorem about matchings in graphs, which is a refinement of Tutte's 1-Factor Theorem. It is also the foundation for Edmonds' famous polynomial algorithm of maximum matchings. This theorem is a powerful accomplishment and it gives much more deeper characterizations to matchings as a subgraph. However, it seems this result has not reached its full potential yet. It is our hope that more applications and appreciation of this theorem are gained in the future.

The structure developed in the proof of Tutte 1-Factor Theorem (i.e., Theorem 1.3.1) is very handy in many proofs of factor related theorems. Typically, in order to prove the existence of 1 -factors, one uses a contra-positive argument by assuming the graph with the given conditions does not have 1-factors. Without loss of generality, we may choose a maximal such graph $H$, i.e., $H$ has no 1 -factor but if any edge is added, the resulting graph does. Such graphs possess many useful properties, we state it in the next theorem. 
Theorem 1.5.1 (see [414]) A graph $H$ of even order is maximal non-1-factorable if and only if it has the following structure:

$G$ consists of vertex-disjoint complete graphs $S, G_{1}, G_{2}, \ldots, G_{k}$ such that $k=|S|+$ 2 , where $G_{1}, \ldots, G_{k}$ are of odd order and every vertex of $G_{i}$ is connected to every vertex of $S$.

Proof. (outline of the proof)

Let $G$ be a maximal non-1-factorable graph of even order. Define $S=\left\{x \mid d_{G}(x)=\right.$ $|V(G)|-1\}$. We complete the proof by showing

(1) $o(G-S)=|S|+2$ (use parity and maximality);

(2) there are no even component in $G-S$;

(3) each component of $G-S$ is a complete graph.

Based on Tutte's 1-Factor Theorem, we can easily obtained formulas for the deficiency and the matching number of a graph.

Theorem 1.5.2 (Berge's Formula) Let def $(G)$ denote the deficiency of a graph $G$. Then

$$
\begin{aligned}
\operatorname{def}(G) & =\max _{S \subseteq V(G)}\{o(G-S)-|S|\}, \\
\mu(G) & =\frac{1}{2}\left(\min _{S \subseteq V(G)}\{|V(G)|-o(G-S)+|S|\}\right) .
\end{aligned}
$$

Proof. Let $d=\operatorname{def}(G)$. At first, we notice that a matching can cover at most $|V(G)|-d$ vertices.

On the other hand, by Tutte's 1-Factor Theorem, the graph $H=G \vee K_{d}$ has a 1-factor. Those edges of a 1-factor of $H$ that belongs to $G$ do not cover at most $d$ vertices of $G$.

Since $\mu(G)=\frac{1}{2}(|V(G)|-\operatorname{def}(G))$, (1.7) follows immediately.

We defined a barrier as a set $S \subseteq V(G)$ such that $o(G-S)=|S|+\operatorname{def}(G)$. So barriers are such sets which reach the maximum in Berge's Formula. If $G$ has a 1 -factor, then $\emptyset$ is a barrier.

From Tutte's 1-Factor Theorem, we see that a graph $G$ of even order has no 1factor if and only if there exists a subset $S$ such that $G-S$ has at least $|S|+2$ odd components. To further understand the structure of arbitrary graphs from the matching point of view, we refine Tutte's condition and introduce a canonical decomposition. This decomposition was studied by Gallai (1963) and Edmonds (1965), independently. 


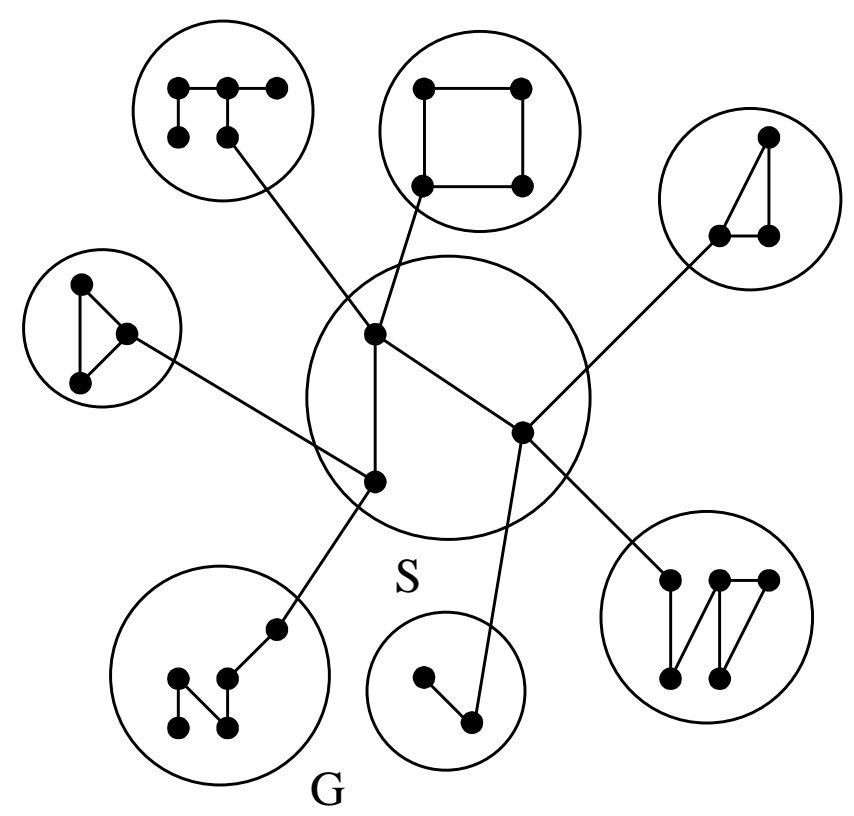

Figure 1.7: A barrier $S$, where $\operatorname{def}(G)=2$ and $\mu(G)=14$

Recall that a graph $G$ is factor-critical if $G-v$ has a 1 -factor for every vertex $v$ of $G$. It is easy to see that if $G$ is factor-critical, then $G$ is of odd order, connected and not a bipartite graph.

For a set $S \subseteq V(G)$, an odd (resp. even) $S$-component is a connected component of the graph $G-S$ of odd (resp. even) cardinality. The total number of vertices in those $S$-components which have no 1-factor is denoted by $\operatorname{Def}(S)$, while the quantity $\operatorname{def}(S)=o(G-S)-|S|$ is called the deficiency of $S$. Directly from the definitions, we have the following two observations.

\section{Observations:}

(1) Every matching in $G$ leaves at least $\operatorname{de} f(S)$ vertices unsaturated;

(2) if a matching $M$ leaves exactly $\operatorname{def}(S)$ vertices unsaturated, then $M$ contains a 1-factor of each even $S$-component, a near-perfect matching of each odd $S$-component, and matches all vertices of $S$ to vertices in distinct odd S-components.

For a vertex set $S$ and a matching $M$, if $M$ has exactly $\operatorname{de} f(S)$ vertices unsaturated (i.e., (2) holds), then $S$ is called a Tutte-Berge set, while $M$ is maximum by (1). The Berge's Formula (1.6) asserts that a Tutte-Berge set $S$ always exists. 
If $|S|+o(G-S) \geq 2$, we denote by $<G, S>$ the bipartite graph obtained from $G$ by deleting all the even $S$-components, contracting each odd $S$-component to a single vertex, and deleting the edges spanned by $S$. For a bipartite graph $H=(A, B)$, the set $A$ satisfies Hall's condition (resp., with positive surplus) if $\left|N_{H}(X)\right| \geq|X|$ (resp., $\left.\left|N_{H}(X)\right|>|X|\right)$ for every non-empty subset $X$ of $A$. Therefore, Hall's Theorem asserts that $H$ has a matching saturated every vertex of $A$ if and only if $A$ satisfies Hall's condition. Finally, we call $S$ a Gallai-Edmonds set with respect to $G$, or a $G-E$ set in short, if $S$ satisfies

(a) all even $S$-components (if any) have a 1 -factor;

(b) all odd $S$-components (if any) are factor-critical;

(c) if $S \neq \emptyset$, then $S$ satisfies Hall's condition with positive surplus in $\langle G, S\rangle$.

Now we are ready to present and prove the famous Gallai-Edmonds Structure Theorem, which is a deep theorem for matchings. It provides a clear structure for graphs without 1-factors and it is the foundation of Edmonds' polynomial algorithm for finding a maximum matching in an arbitrary graph. It deserves more attention than its current status in authors' opinion. The proof used here is given by Kotlov [323] and it derives Gallai-Edmonds Structure Theorem from Hall's Theorem.

Theorem 1.5.3 (Gallai-Edmonds Structure Theorem) Let $G$ be a graph. Then

(i) there exists a Gallai-Edmonds set $S$;

(ii) $S$ is a Tutte-Berge set. That is, every maximum matching of $G$ contains a nearperfect matching of each odd S-component, a 1-factor of each even $S$-component, and matches all vertices of $S$ to vertices in distinct $S$-components;

(iii) the underlying vertex set of all odd $S$-components is the set $D(G)$ of the vertices left unsaturated by at least one maximum matching of $G$, while $S$ is the neighborhood of $D(G)$. In particular, $G$ has a unique Gallai-Edmonds set.

Proof. (i) Among all vertex subsets of $G$ with maximum deficiency, let $S$ have minimum $\operatorname{Def}(S)$ and $|S|=s$. We use induction on $|G|$ to show that $S$ is a G-E set.

Suppose that $C$ is an even $S$-component without 1-factors. For any fix $v \in V(C)$ and let $S^{\prime}=S \cup\{v\}$. Then $\operatorname{def}\left(S^{\prime}\right) \geq \operatorname{def}(S)$ while $\operatorname{Def}\left(S^{\prime}\right)<\operatorname{Def}(S)$. This contradiction shows that $S$ satisfies (a).

Suppose that $v$ is a vertex in an odd $S$-component $C$ such that $C-v$ has no 1-factor. By induction hypothesis, the graph $H=C-v$ has a G-E set $T$. In particular, $\operatorname{def}_{H}(T) \geq 2$. Set $S^{\prime}=S \cup T \cup\{v\}$. Then $\operatorname{def}\left(S^{\prime}\right)=o\left(G-S^{\prime}\right)-\left|S^{\prime}\right|=$ $(o(G-S)+o(H-T)-1)-(s+|T|+1)=\operatorname{def}(S)+\operatorname{def}_{H}(T)-2 \geq \operatorname{def}(S)$ while $\operatorname{Def}\left(S^{\prime}\right)<\operatorname{Def}(S)$, a contradiction. Thus, $S$ satisfies (b). 
Suppose that $X$ is a smallest non-empty subset of $S$ violating Hall's condition with positive surplus in $\langle G, S\rangle$. Set $S^{\prime}=S-X$. If $X$ consists of a single vertex with no neighbors in $\langle G, S\rangle$, then $\operatorname{def}\left(S^{\prime}\right)>\operatorname{def}(S)$, which is a contradiction. Otherwise, $X$ satisfies Hall's condition in $\langle G, S\rangle$. By (b) and Hall's Theorem, $X$ is contained in an even $S^{\prime}$-component with a 1-factor (this even component is spanned by $X$ and the $|X|$ odd $S$-components neighboring with $X$ in $G)$. Consequently, $\operatorname{def}\left(S^{\prime}\right)=\operatorname{def}(S)$ and $\operatorname{Def}\left(S^{\prime}\right)<\operatorname{Def}(S)$. Thus, $S$ satisfies (c).

Let now $S$ be a G-E set, and $v$ a fixed vertex in an odd $S$-component $C$. By (c) and Hall's Theorem, $S$ can be matched to vertices in $s$ distinct odd $S$-components different from $C$. By (a) and (b), this matching can be extended by (near-)perfect matchings of the $S$-components to obtain a matching $M$ avoiding $v$. Since $M$ leaves $\operatorname{def}(S)$ vertices unsaturated, it is maximum, and (ii) and (iii) follow immediately.

From Theorem 1.5.3, the G-E set plays an important role in the structure of a graph. We can actually determine this G-E set explicitly as follow: for a graph $G$, denote by $D(G)$ the set of all vertices in $G$ which are not saturated by at least one maximum matching of $G$. Let $A(G)$ be the neighbor set of $D(G)$, i.e., the set of vertices in $V(G)-D(G)$ adjacent to at least one vertex in $D(G)$. Finally let $C(G)=V(G)-D(G)-A(G)$. Clearly, this partition is well-defined for every graph and does not rely on the choices of maximum matchings. In fact, the set $A(G)$ is our G-E set. The partition $(D(G), A(G), C(G))$ is called a canonical decomposition.

This partition of $V(G)$ is also refinement of Tutte's 1-factor Theorem, $A(G)$ can be thought as a Tutte-set $S$ (which is a barrier), $D(G)$ is the union of the odd components of $G-S$ and $C(G)$ is the union of the even components of $G-S$. As an example, Figure 1.8 shows the canonical decomposition $(D(G), A(G), C(G))$.

With the canonical decomposition $(D(G), A(G), C(G))$, we can restate Theorem 1.5.3 in the following form, which is the usual formation in most literatures.

Theorem 1.5.4 (Gallai-Edmonds Structure Theorem) Let $G$ be a graph and let $D(G), C(G)$ and $A(G)$ be the vertex-partition defined above. Then

(i) the components of the subgraph induced by $D(G)$ are factor-critical;

(ii) the subgraph induced by $C(G)$ has a 1-factor;

(iii) if $M$ is any maximum matching of $G$, it contains a near-perfect matching of each component of $D(G)$, a 1-factor of each component of $C(G)$ and matches all vertices of $A(G)$ with vertices in distinct components of $D(G)$;

(iv) the bipartite graph obtained from $G$ by deleting the vertices of $C(G)$ and the edges spanned by $A(G)$ and by contracting each component of $D(G)$ to a single vertex has positive surplus (as viewed from $A(G)$ ); 


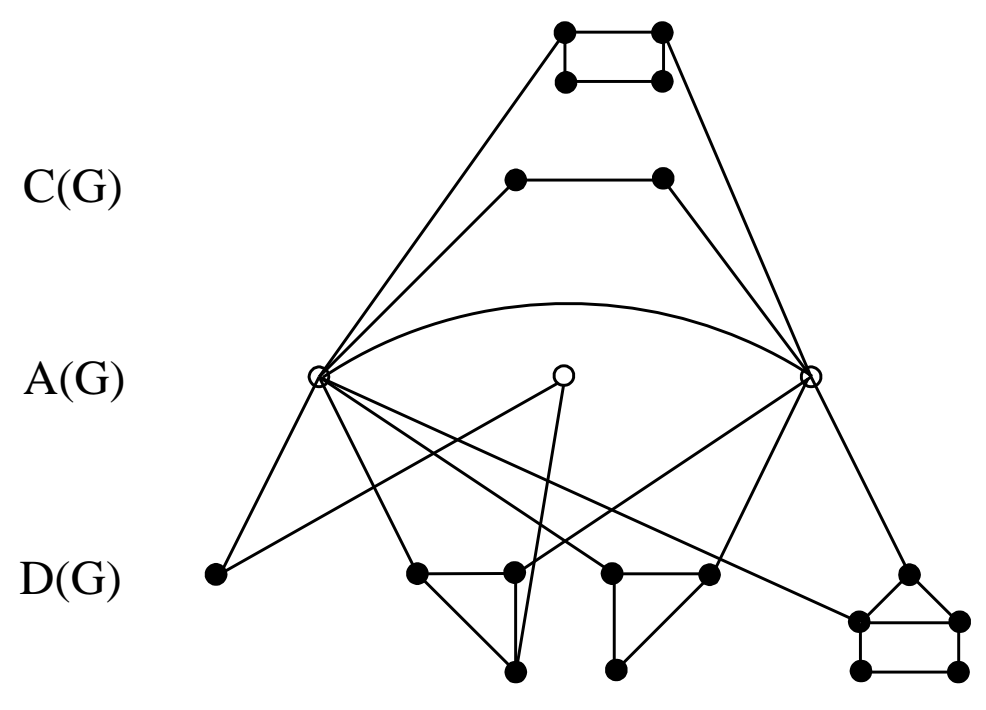

Figure 1.8: The canonical decomposition $(D(G), A(G), C(G))$

(v) the size of maximum matching is $\frac{1}{2}(|V(G)|-\omega(D(G))+|A(G)|)$.

Note: For a bipartite graph $G=(X, Y)$, the surplus of a set $S \subseteq X$ is defined by $|N(S)|-|S|$. The surplus of $G$ is the minimum surplus of all non-empty subset of $X$.

Clearly, Theorem 1.5.4 provides more information than Tutte's 1-Factor Theorem. In fact, the latter can be easily derived from former. To see this, note that since $o(G-S) \leq|S|$ holds for all $S \subseteq V(G)$, in particular $o(G-A(G)) \leq|A(G)|$ and then substituting this into the formula in (v), we have $\frac{1}{2}(|V(G)|-\omega(D(G))+|A(G)|) \geq$ $\frac{1}{2}|V(G)|$. Thus $G$ has a 1-factor. Similarly, let $S=A(G)$ and (v) yields equality of Berge's Formula.

In fact, the structure provided by Gallai-Edmonds Theorem has many other important consequences as well. Many of the results in this chapter are consequences of this powerful theorem.

Remark 1.5.1. (1) From the part (v), it is immediate that the deficiency of $G$, $\operatorname{def}(G)=\omega(D(G))-|A(G)|$; that is, it is the number of components of $D(G)$ left unmatched into $A(G)$ by an arbitrary maximum matching of $G$.

(2) If $G$ has no 1-factor, then every edge incidents with a vertex of $D(G)$ lies in some maximum matching of $G$. To see this, let $u \in D(G)$ and $u v$ be any edge incidents to $u$. By the definition of $D(G)$, there is a maximum matching missing $u$. Hence by the maximality of $M$, there is an edge $v w$ in $M$ which covers $v$. But then $M-v w \cup u v$ is the desired matching. 
It follows from (iv) that no edge induced by $A(G)$ or connecting $A(G)$ to $C(G)$ belongs to any maximum matching. The edges in the subgraph induced by $C(G)$ show a much more complicated behavior in this respect and this problem will be discussed later in Chapter 2.

(3) If $G$ itself is a factor-critical graph, then we have a degenerate case of the canonical decomposition; namely, $D(G)=V(G)$, so $A(G)=C(G)=\emptyset$.

(4) If $G$ has a 1-factor, we have another degenerate case; namely, $D(G)=\emptyset$, so $A(G)=\emptyset$ and hence $C(G)=V(G)$.

(5) If $G$ is a bipartite graph with bipartition $(X, Y)$ and if it has positive surplus (from $X$ ), then $A(G)=X$ and $D(G)=Y$. To see this, note that $\mu(G)=|X|$ and Hall's Theorem implies that $G-v$ has a maximum matching of $X$ into $Y-v$ for every $v \in Y$. Hence $Y=D(G)$ and $X=A(G)$.

Thus the Gallai-Edmonds canonical decomposition is trivial in three cases: factor-critical graphs, graphs with 1-factors and bipartite graphs with positive surplus. On the other hand, it tells us that every graph can be built up from graphs of these three types.

Besides the properties mentioned in Theorem 1.5.4 and Remark 1.5.1, the sets $D(G), C(G)$ and $A(G)$ have other interesting features as well. The next assertion was used as a lemma to prove Theorems 1.5.3 and 1.5.4 in several literatures. Even though we do not need it for the proof of Theorems 1.5.3, the assertion has its own merit to be mentioned.

Theorem 1.5.5 (Stability Lemma, see [414]) Let $G$ be a graph and let $D(G), C(G)$ and $A(G)$ be defined above. Then

(i) for any $u \in A(G)$, then $A(G-u)=A(G)-u, C(G-u)=C(G)$, and $D(G-u)=$ $D(G)$;

(ii) for any $u \in C(G)$, then $A(G-u) \supseteq A(G), C(G-u) \subseteq C(G)-u$, and $D(G-u) \supseteq D(G)$;

(iii) for any $u \in D(G)$, then $A(G-u) \subseteq A(G), C(G-u) \supseteq C(G)$, and $D(G-u) \subseteq$ $D(G)-u$.

Proof. We only give the detailed proof of part (i) here. For parts (ii) and (iii), it can be proved similarly (see [414]).

(i) From the definitions of $D(G), C(G)$ and $A(G)$, it is clear that we need only to show $D(G-u)=D(G)$.

Let $M$ be a maximum matching of $G$ and thus $\mu(G)=|M|$. Since $u \notin D(G)$, then $M$ covers $u$. Hence $\mu(G-u)=\mu(G)-1$. Moreover, since $M$ is a maximum matching of $G, M-u$ is a maximum matching of $G-u$. 
To see $D(G) \subseteq D(G-u)$, choose any $v \in D(G)$, let $M_{v}$ be a maximum matching of $G$ which misses $v$. Then $M_{v}-u$ is a maximum matching of $G-u$ and moreover, $M_{v}-u$ misses $v$ too, so $v \in D(G-u)$. Thus $D(G) \subseteq D(G-u)$.

To see $D(G-u) \subseteq D(G)$, choose any $v \in D(G-u)$. Then there is a maximum matching $M^{\prime}$ of $G-u$ which misses $v$. Let $w$ be any vertex in $D(G)$ adjacent to $u$ in $G$ and let $M$ be a maximum matching of $G$ misses $w$. If $M$ misses $v$, then $v \in D(G)$ follows, so we assume that $M$ covers $v$. Consider $M \cup M^{\prime}$. By definition, $M^{\prime}$ avoids $v$. Thus the component of $M \cup M^{\prime}$ covering $v$ must be a path $P$ starting at $v$ with an edge of $M-M^{\prime}$.

Suppose $P$ ends with an edge of $M^{\prime}$. Then $M \triangle P$ is a new matching $M^{\prime \prime}$ in $G$ missing $v$. Moreover, $\left|M^{\prime \prime}\right|=|M|$, so $M^{\prime \prime}$ is a maximum matching and $v \in D(G)$. Otherwise, $P$ ends with an edge of $M$. Let $M^{\prime \prime \prime}=M^{\prime} \triangle P$. Then $M^{\prime \prime \prime}$ is a matching and $\left|M^{\prime \prime \prime}\right|>|M|$. So $M^{\prime \prime \prime} \nsubseteq E(G-u)$ by the maximality of $M^{\prime}$. Thus $P$ must end at $u$. But now $M \triangle E(P \cup u w)$ is a maximum matching of $G$ avoiding $v$ and thus $v \in D(G)$.

For bipartite graphs, we have the following structural theorem.

Theorem 1.5.6 (see [414]) Let $G=\left(U_{1}, U_{2}\right)$ be a connected bipartite graph, and let $C_{1}=C(G) \cap U_{1}$ and $C_{2}=C(G) \cap U_{2}$. Then the following statements hold

(i) $G[D(G)]$ is an independent set $G$;

(ii) $G[C(G)]$ has a 1-factor, and $\left|C_{1}\right|=\left|C_{2}\right|$;

(iii) every maximum matching $M$ in $G$ consists of a 1-factor of $G[C(G)]$ and a matching in $G[A(G) \cup D(G)]$ saturating $A(G)$;

(iv) both $A(G) \cap C_{1}$ and $A(G) \cap C_{2}$ are minimum vertex covers of $G$;

(v) both $D(G) \cap C_{1}$ and $D(G) \cap C_{2}$ are maximum independent subsets of $G$.

As an application of Gallai-Edmonds Structure Theorem, we prove the following result regarding the connectivity of allowed edges. More applications of Theorem 1.5.4 can be found in Section 5.1.

Theorem 1.5.7 (see [414]) Let $G$ be a connected graph with 1-factors. If $C(G-x)=\emptyset$ for all $x \in V(G)$, then all allowed edges form a connected spanning subgraph of $G$.

Proof. Suppose, by the contrary, that there exists a partition of $V(G)$, say $\left(V_{1}, V_{2}\right)$ where $V_{1} \neq \emptyset \neq V_{2}$, such that all edges of $E\left(V_{1}, V_{2}\right)$ are not allowed, i.e., for any 1-factor $M$, we have $M \cap E\left(V_{1}, V_{2}\right)=\emptyset$. Thus $M$ must match $V_{i}$ onto itself and so $\left|V_{i}\right|$ is even $(i=1,2)$.

Claim 1. There exists a subset $S$ such that $|S| \geq 2$ and $o(G-S)=|S|$. 
Let $v_{1} v_{2} \in E\left(V_{1}, V_{2}\right)$, where $v_{1} \in V_{1}$ and $v_{2} \in V_{2}$. Then $v_{1} v_{2}$ is not allowed, that is, $G^{\prime}=G-\left\{v_{1}, v_{2}\right\}$ has no 1-factor. From 1-Factor Theorem, there exists a subset $S^{\prime} \subseteq V\left(G^{\prime}\right)$ such that $o\left(G^{\prime}-S^{\prime}\right)>\left|S^{\prime}\right|$ or $o\left(G^{\prime}-S^{\prime}\right) \geq\left|S^{\prime}\right|+2$ by parity. Let $S=S^{\prime} \cup\left\{v_{1}, v_{2}\right\}$. Then $o(G-S)=o\left(G^{\prime}-S^{\prime}\right) \geq\left|S^{\prime}\right|+2=|S|$. On the other hand, since $G$ has 1 -factors, we have $o(G-X) \leq|X|$ for any subset $X \subseteq V(G)$. Thus $o(G-S)=|S|$ or $S$ is a barrier.

Without loss of generality, we assume that $S$ is a maximal barrier.

Claim 2. Every edge $e=x y$ with $x \in S$ and $y \notin S$ is allowed in $G$.

Consider the graph $G-x$. Since $\operatorname{def}(G)=0$, so $\operatorname{def}(G-x)=1$. Because of $o(G-S)=|S|, x \in S$ and $\operatorname{def}(G-x)=1$, every maximum matching of $G-x$ saturates every vertex of $S-x$. By the definition of $D(G-x)$, so $S-x \subseteq A(G-x) \cup C(G-x)=$ $A(G-x)$ as $C(G-x)=\emptyset$. On the other hand, by Stability Lemma (i.e., Theorem 1.5.5), $A(G-x)=A(G)-x$ and thus $A(G-x) \cup x=A(G)$ is a barrier in $G$. By the maximality of $S, A(G-x) \cup x \subseteq S$. Thus

$$
S=A(G-x) \cup x .
$$

Since $y \notin S$, by (1.8), $y \in D(G-x) \cup C(G-x)=D(G-x)$ as $C(G-x)=\emptyset$ again. So there exists a maximum matching $M_{x}$ of $G-x$ missing $y$ but saturating all other vertices of $G-x$. But then $M_{x} \cup\{x y\}$ is a 1-factor of $G$, i.e., $x y$ is an allowed edge of $G$.

Claim 3. $G-S$ has no odd component joined to both $S \cup V_{1}$ and $S \cap V_{2}$.

Suppose, to the contrary, that $H$ is such a component. Let $u v$ be an edge with $u \in V(H)$ and $v \in S \cap V_{1}$. By Claim 2, there exists a 1-factor $F$ of $G$ containing $u v$. Thus $u \in V_{1}$ by the definition of $V_{1}$.

Since $|V(G)|$ is even, we have $D(G-v) \neq \emptyset$ but $C(G-v)=\emptyset$. Let $e_{v}$ be an edge of $F$ which is incident to $v$. Then $F-e_{v}$ is a maximum matching of $G-v$. Moreover, $F-e_{v}$ saturates all vertices of $A(G-v)$ and has at most one edge joining each odd component of $D(G-v)$ (of which $H$ is one) to $A(G-v)$. Recall $S=A(G-v) \cup v$, upon reinstalling $v$ and $e_{v}$ we have exactly one edge of $F$ joining $S$ to each component of $G-S$ and in particular, exactly one edge of $F$ joining $H$ to $S$. So $E(H) \cap F$ is a near-perfect matching of $H$. Thus $E\left(H-V_{2}\right) \cap F$ is a near-perfect matching of $H-V_{2}$, and so $\left|V(H) \cap V_{1}\right|$ is odd. Similarly, $\left|V(H) \cap V_{2}\right|$ is odd and hence $|V(H)|$ is even, a contradiction.

By Claims 2 and 3, all odd components of $G-S$ are joined to $S \cap V_{1}$ or $S \cap V_{2}$, but not both. For $i=1,2$, let $a_{i}$ be the number of odd components of $G-S$ joined to $S \cap V_{i}$. Then $a_{1}+a_{2}=o(G-S)=|S|$. Without loss of generality, assume $a_{1} \leq\left|S \cap V_{1}\right|$. Let $x \in S \cap V_{2}$. Then, from (1.8), $A(G-v)$ has a subset of $k=\left|S \cap V_{1}\right|$ vertices joined to at most $k$ odd components of $(G-x)-A(G-x)$, which contradicts to the part (iv) of Gallai-Edmonds Structure Theorem.

The proof is complete. 
Kelmans [314] studied the induced star packing problem as a natural generalization of the matching problem and obtained a Gallai-Edmonds type of structure theorem for induced star packing and also included Tutte's 1-Factor Theorem, Berge's Formula, the matching matroid theorem as special cases.

Pulleyblank [503] extend Theorem 1.5.4 to fractional matchings. We will provide more details in Section 8.1.

\subsection{Number of 1-factors}

Let $\Phi(G)$ denote the number of 1-factors in a graph $G$. One of focuses in the study of matching theory is to determine the function $\Phi(G)$. However, it seems very unlikely that a formula or an efficient algorithm for computing $\Phi(G)$ will ever be found. In particular, Valiant (1979) proved that the problem of determining $\Phi(G)$ is NP-hard, even when $G$ is bipartite. This left very little room for finding the exact value of $\Phi(G)$. Naturally, the next move is to find a lower bound for $\Phi(G)$.

Let's first present a few classical results in this direction.

Theorem 1.6.1 (Hall (1948)) Let $G=(X, Y)$ be a bipartite graph with a 1-factor and $d(x) \geq k$ for every $x \in X$. Then $\Phi(G) \geq k$ !.

Theorem 1.6.2 (Beineke and Plummer (1967)) Let $G$ be a 2-connected graph with a 1-factor. Then $\Phi(G) \geq 2$.

Theorem 1.6.3 (Zaks (1970)) Let $G$ be a $k$-connected graph with a 1-factor $(k \geq 2)$. Then $\Phi(G) \geq k !$ !. In particular, $\Phi\left(K_{n}\right)=(n-1) ! !=(n-1)(n-3) \ldots 1$. The bounds are sharp when $k$ is odd.

Theorem 1.6.4 Let $G$ be a connected graph of order $n$ with a unique 1-factor. Then

(a) $G$ has a bridge belonging to the 1-factor;

(b) $G$ contains a vertex of degree $\leq\left\lfloor\log _{2}(n+1)\right\rfloor$; and

(c) $|E(G)| \leq \frac{1}{4} n^{2}$.

Note that, in the above theorem, (a) is from Kotzig (1959); (b) is due to Lovász (1972) and (c) is from Hetyei (unpublished manuscript, see [406]).

Theorem 1.6.5 (Lovász [406]) Let $G$ be a k-connected graph with a 1-factor and $G$ is not bicritical. Then $\Phi(G) \geq k$ !

An immediate consequence of Theorem 1.6.5 is that a $k$-connected graph $G$ with 1-factors has $\Phi(G) \geq k$ ! or $G$ is bicritical. Bicritical graphs are a family of important graphs, and it plays a unreplaceable role in the study of matchings, for example, it can be used to develop a decomposition theory for elementary graphs (ref. Section 5.2) 
and it can also be used to determine the dimension of 1-factors lattice (ref. Section $5.4)$.

From the definition of bicriticality, it seems that this family of graphs are very rich in 1-factors. However, bicritical graphs is somewhat counterintuitive. In fact, bicritical graphs contain much less 1-factors than other graphs and is more difficult to bound $\Phi(G)$ than most other graphs, as shown in the next theorem. This theorem has a weaker condition, 2-connected and $\delta(G) \geq k$, than Theorem 1.6.5.

Theorem 1.6.6 (Mader (1976)) Let $G$ be a 2-connected graph with $\delta(G) \geq k$ and have a 1-factor. Then either

(a) there are at least $k$ ! 1-factors in $G$ (i.e., $\Phi(G) \geq k$ !), or

(b) $G$ is bicritical. In this case, $\Phi(G) \geq|V(G)| / 2+1$.

The investigation of lower bound for general graphs is still ongoing. On the other hand, the lower bounds for some special families of graphs are also researched. For example, Lovász and Plummer [415] obtained the best possible lower bound $\Phi(H)$ for Halin graphs $H$ (see Theorem ?? for the details).

It has been shown that one can bound $\Phi(G)$ below by a certain matrix function called Pfaffian. In the case when $G$ is planar, the Pfaffian matrices can be used to exactly compute $\Phi(G)$ in polynomial time. (The definition of Pfaffian orientation can be found in Section 5.4)

Now we turn to the problem of finding a lower bound for the number of nearperfect matchings in a factor-critical graphs. The following result was obtained by Pulleyblank (1973) and he used linear programming technique to prove it. Here we present a short proof from [414].

Theorem 1.6.7 (Pulleyblank [502]) Every 2-connected factor-critical graph $G$ contains at least $|E(G)|$ near-perfect matchings.

Proof. Since $G$ is a 2-connected factor-critical graph, from Theorem ??, so $G$ can be expressed as $G=P_{0}+P_{1}+P_{2}+\cdots+P_{m}$, where $P_{0}$ is an odd cycle and each $P_{r}(1 \leq r \leq m)$ is an open path of odd length joining two different vertices of $P_{0}+P_{1}+\cdots+P_{r-1}$. Denote that $G_{r}=P_{0}+P_{1}+\cdots+P_{r}$. Then $G_{r}$ is factor-critical.

We use induction on $m$. If $m=0$, the result clearly holds. Suppose the result holds for $r \geq 0$. We consider $G_{r+1}=G_{r}+P_{r+1}$, which is a 2-connected factor-critical graph. Let $P_{r+1}=x u_{1} \ldots u_{l-1} y$, where $l=1$ or $l \geq 3$.

If $F$ is any near-perfect matching of $G_{r}$, then $F^{\prime}=F \cup\left\{u_{1} u_{2}, \ldots, u_{l-2} u_{l-1}\right\}$ is a near-perfect matching of $G_{r+1}$. By induction hypothesis, there are $\left|E\left(G_{r}\right)\right|=$ $\left|E\left(G_{r+1}\right)\right|-l$ near-perfect matchings in $G_{r+1}$.

Since $G_{r}$ is factor-critical, for each $u_{i}(1 \leq i \leq l-1)$ we can easily find a 1 -factor $F_{u_{i}}$ of $G_{r+1}-u_{i}$. So we obtain $l-1$ more near-perfect matchings of $G_{r+1}$. Note that 
each near-perfect matching $F_{u_{i}}$ saturates only one of $x$ and $y$ with an edge of $P_{r+1}$. Next we construct the last near-perfect matching of $G_{r+1}$, which saturates both of $x$ and $y$ with the edges of $P_{r+1}$. Let $F_{x}$ be a 1-factor of $G_{r}-x$. Then $F_{x}$ must cover $y$ with an edge $y z \in E\left(G_{r}\right)$. Therefore $F^{\prime \prime}=\left(F_{x}-\{y z\}\right) \cup\left\{x u_{1}, u_{2} u_{3}, \ldots, u_{l-1} y\right\}$ is a 1-factor of $G_{r+1}-z$ and thus the desired near-perfect matching of $G_{r+1}$.

We complete the induction.

Now we divert our attention from determining the value of $\Phi(G)$ to study of the structure of graphs which are sensitive to $\Phi(G)$. The most results in this area are due to Lovász [406]. Reader may choose to read this part after Chapter 5 (in particular, Section 5.1) since some terms and results are given in details there.

A graph $G$ with a 1-factor is $\Phi$-maximal if $\Phi(G \cup e)>\Phi(G)$ for any edge $e \notin E(G)$, i.e., the graph $G \cup e$ has more 1-factors than $G$. Note that a 1-extendable graph is $\Phi$-maximal if and only if it is bicritical. Every graph with 1-factors is contained in a $\Phi$-maximal graph because we can add edges to a graph as long as no new 1factor are generated. Many important graph-theoretic properties are preserved by this procedure.

A subset $S \subseteq V(G)$ is called extreme if $\operatorname{def}(G-S)=\operatorname{def}(G)+|S|$. From the definition of extreme sets, it is easy exercises to see the following properties of extreme sets.

Lemma 1.6.1 Let $G$ be a graph. Then

(a) every subset of an extreme set is extreme;

(b) if $S$ is an extreme set and $S \subseteq T$, then $T-S$ is an extreme set too.

Below, we characterize $\Phi$-maximal graphs.

Theorem 1.6.8 Let $G$ be any graph with 1-factors. Then $G$ is $\Phi$-maximal if and only every extreme set in $G$ induces a complete graph, all edges of which are forbidden in $G$.

Proof. Assume that $G$ is $\Phi$-maximal. Let $X$ be an extreme set in $G$ and $\{x, y\} \subseteq X$. By Lemma 1.6.1, $\{x, y\}$ is an extreme set of $G$.

Suppose $x$ and $y$ are not adjacent in $G$. Then $\operatorname{def}((G \cup x y)-x-y)=\operatorname{def}(G-$ $x-y)=\operatorname{def}(G)+2$ since $\{x, y\}$ is extreme in $G$. But $\operatorname{def}(G \cup x y)=\operatorname{def}(G)=0$, so we have $\operatorname{def}((G \cup x y)-x-y)=\operatorname{def}(G \cup x y)+2$ and thus $\{x, y\}$ is extreme in $G \cup x y$. Therefore, $x y$ is forbidden in $G \cup x y$ and so $G$ is not $\Phi$-maximal, a contradiction. Then we must have $x y \in E(G)$ and thus $x y$ is forbidden in $G$.

Conversely, suppose $G$ is not $\Phi$-maximal and thus there are two vertices $x, y \in$ $V(G)$ with $x y \notin E(G)$, such that $G$ and $G \cup x y$ have the same 1-factors. Thus $x y$ is forbidden in $G \cup x y$ and $\{x, y\}$ is extreme in $G \cup x y$. So $\operatorname{def}(G-x-y)=$ $\operatorname{def}((G \cup x y)-x-y)=\operatorname{def}(G \cup x y)+2=0+2=\operatorname{def}(G)+2$ and thus $\{x, y\}$ is 
extreme in $G$. But this contradicts the hypothesis that $x y$ must be a forbidden edge of $G$.

In addition, if a $\Phi$-maximal graph is also elementary (i.e., allowed edges form a connected subgraph), then Theorem 1.6.8 yields the following.

Theorem 1.6.9 If $G$ is a $\Phi$-maximal elementary graph, then the forbidden edges of $G$ constitute vertex-disjoint complete subgraphs induced by the classes of $\mathcal{P}(G)$.

The next result shows us that the Construction of general elementary graphs discussed in Section 5.2 is "more unique" when graphs are $\Phi$-maximal.

Theorem 1.6.10 Let $G$ be a $\Phi$-maximal elementary graph and let $G^{*}$ be the graph obtained by contracting each class $S$ of $\mathcal{P}(G)$ to a vertex $t_{S}$. Then

(a) the block of $G^{*}$ (i.e., the maximal 2-connected subgraph of $G^{*}$ ) are bicritical;

(b) the number of blocks of $G^{*}$ containing $t_{S}$ is exactly $|S|$;

(c) if we build $G$ by iterating the construction of Theorem ??, then the initial bicritical graphs must be precisely the blocks of $G^{*}$, and

(d) the elementary bipartite graphs used in the last steps of the Construction of $G$ are uniquely determined in the sense that they must be of the form $G_{S}^{\prime}$, for some $S \in \mathcal{P}(G)$.

Now we proceed to the main result of this section - Cathedral Theorem - for all $\Phi$-maximal non-elementary graphs. First, we introduce Cathedral Construction, which was studied by Kotzig (1960):

Cathedral Construction. Let $G_{0}$ be any $\Phi$-maximal elementary graph. To each class $S \in \mathcal{P}\left(G_{0}\right)$ assign an (already constructed) $\Phi$-maximal graph $G_{S}$ or the empty set. For each $S \in \mathcal{P}\left(G_{0}\right)$ join every vertex of $S$ to every vertex of $G_{S}$. If $G_{S}$ is not empty, we call the subgraph $G_{S}$ the tower over $S$ and call $S$ the foundation of that tower. (Note that a tower and its foundation are vertex-disjoint.)

Now we can state Cathedral Theorem, but the proof is long and involved and we omit it.

Theorem 1.6.11 (Cathedral Theorem)

(a) Every graph $G$, built up by iterating Cathedral Construction using smaller $\Phi$ maximal graphs, is itself $\Phi$-maximal;

(b) the allowed edges of $G$ are precisely those edges which are allowed in one of the elementary graphs used in one of the steps. 

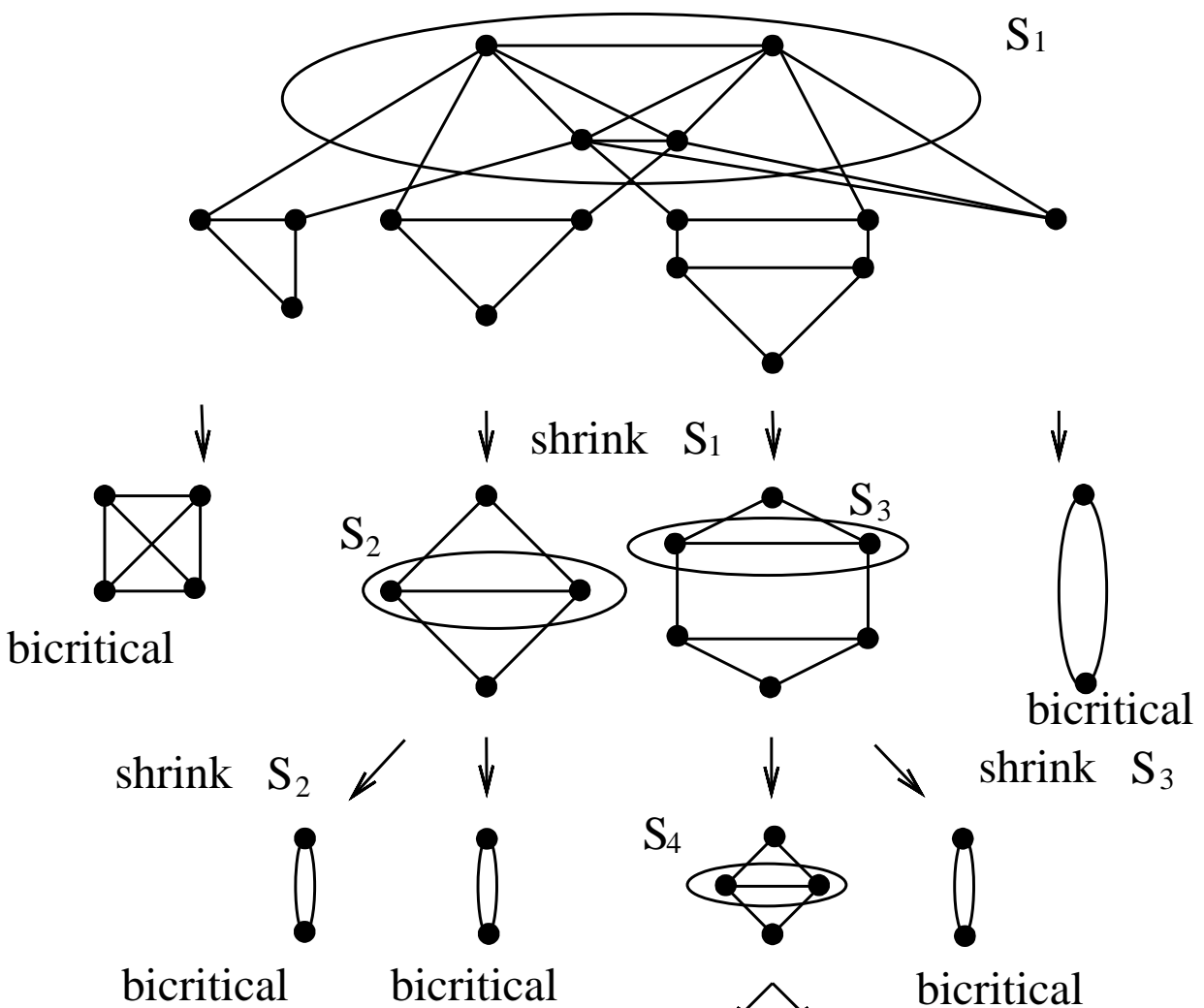

$\searrow$ shrink $\mathrm{S}_{3}$

bicritical

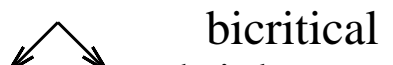
shrink $\mathrm{S}_{4}$

bicritical

Figure 1.9: Construction of a $\Phi$-maximal elementary graph

(c) Conversely, if $G$ is any $\Phi$-maximal graph, it can be built up using Cathedral Construction starting with a $\Phi$-maximal elementary graph $G_{0}$ and a collection of $\left|\mathcal{P}\left(G_{0}\right)\right|$ smaller $\Phi$-maximal graphs (some perhaps empty) already constructed. The graph $G_{0}$ may be uniquely described as the subgraph of $G$ induced by those vertices of $G$ which, for each $x \in V(G)$, does not lie in $C(G-x)$.

An illustration of Cathedral structure is shown in Figure 1.10.

Determining $\Phi(G)$ for a graph $G$ is an extremely difficult task, even for a special class of graphs, e.g., bipartite graph. One of important tools in studying $\Phi(G)$ of bipartite graphs is to use the permanent of incident matrix of $G$. There is a direct correspondence between binary $n \times n$ matrices and balanced bipartite graphs of order $2 n$. So to compute the exact value of $\Phi(G)$ is equivalent to find the permanent 


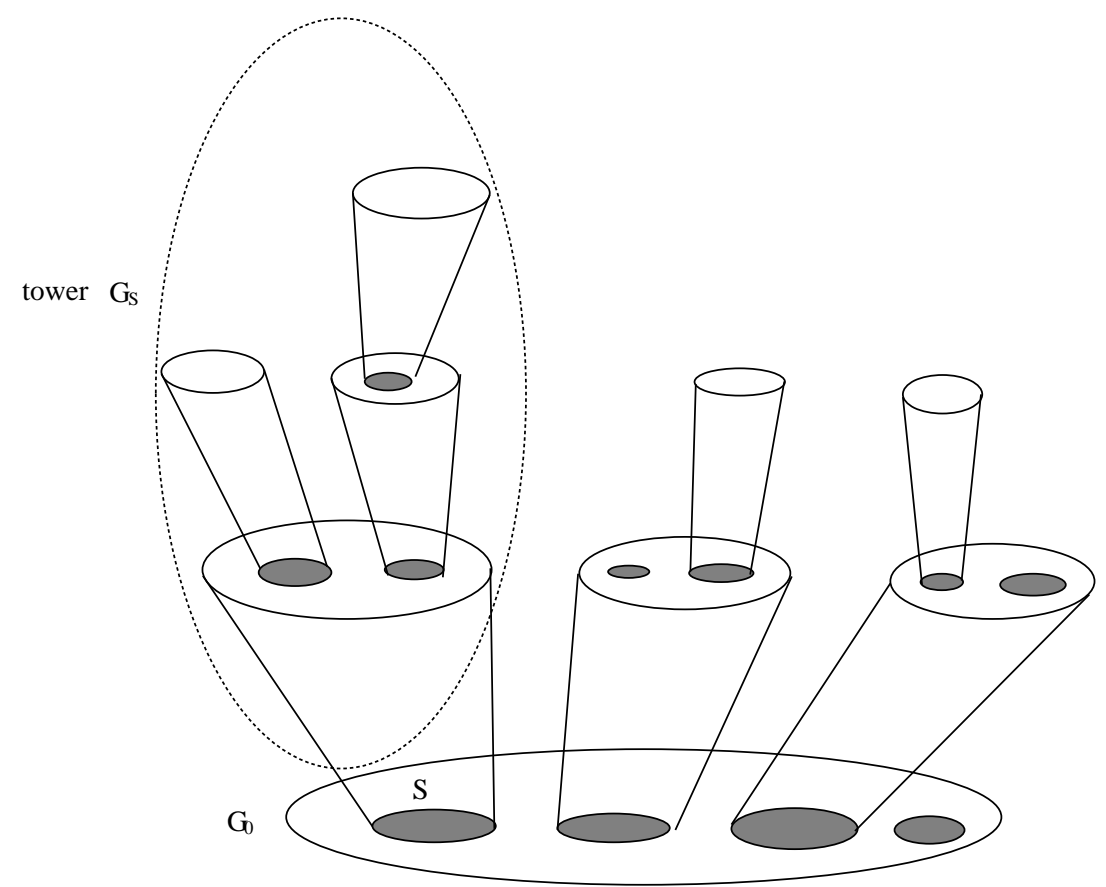

Figure 1.10: A Cathedral Construction

of corresponding matrices. Even though to determine the permanent of matrices is again a $N P$-complete problem, however the equivalence between the permanent and $\Phi(G)$ leads to the discovery of a good non-trivial lower bound for $\Phi(G)$ when $G$ is bipartite and regular. The lower bound and upper bound are obtained by Schrijver and Brègman, respectively.

Theorem 1.6.12 (Schrijver (1998); Brègman (1973)) Let $G$ be a k-regular bipartite graph on $2 n$ vertices. Then

$$
\left(\frac{(k-1)^{k-1}}{k^{k-2}}\right)^{n} \leq \Phi(G) \leq(k !)^{n / k}
$$

As a conclusion of this section, as well as the chapter, we pose a conjecture on $\Phi(G)$ by Lovász and Plummer (1989).

Conjecture 1.6.1 (Lovász and Plummer [418]) Let $G$ be a 2-edge-connected cubic graph $G$. Then there exists a constant $c>1$ such that $\Phi(G) \geq c^{n}$.

There are some partial results known for this conjecture. For example, Voorhoeve (1979) showed that if $G$ is a cubic bipartite graph on $2 n$ vertices, then $\Phi(G) \geq(4 / 3)^{n}$. 


\section{Bibliography}

[1] P. Adams, M. Mahdian and E. S. Mahmoodian, On the forced matching numbers of bipartite graphs, Discrete Math. 281 (2004) (1-3) 1-12.

[2] L. Addario-Berry, R. E. L. Aldred, K. Dalal and B. A. Reed, Vertex colouring edge partitions, J. Combin. Theory Ser. B 94 (2005) (2) 237-244.

[3] L. Addario-Berry, K. Dalal, C. McDiarmid, B. Reed and A. Thomason, Vertexcolouring edge-weightings, Combinatorica 27 (2007) (1) 1-12.

[4] L. Addario-Berry, K. Dalal and B. A. Reed, Degree constrained subgraphs, Discrete Appl. Math. 156 (2008) (7) 1168-1174.

[5] R. Aharoni, A generalization of Tutte's 1-factor theorem to countable graphs, J. Combin. Theory Ser. B 37 (1984) (3) 199-209.

[6] R. Aharoni, Infinite matching theory, Discrete Math. 95 (1991) (1-3) 5-22.

[7] M. Aigner and S. Brandt, Embedding arbitrary graphs of maximum degree two, J. London Math. Soc. (2) 48 (1993) (1) 39-51.

[8] A. Ainouche, Quasi-claw-free graphs, Discrete Math. 179 (1998) (1-3) 13-26.

[9] J. Akiyama, D. Avis and H. Era, On a $\{1,2\}$-factor of a graph, TRU Math. 16 (1980) (2) 97-102.

[10] J. Akiyama, G. Exoo and F. Harary, Covering and packing in graphs IV: Linear arboricity, Networks 11 (1981) (1) 69-72.

[11] J. Akiyama and M. Kano, Almost-regular factorization of graphs, J. Graph Theory 9 (1985) (1) 123-128.

[12] J. Akiyama and M. Kano, Factors and factorizations of graphs - a survey, J. Graph Theory 9 (1985) (1) 1-42.

[13] J. Akiyama and M. Kano, Factors and Factorizations of Graphs, Ver. 1.0, June 2007 (in press). 
[14] J. Akiyama and M. Kano, Path factors of a graph, in Graphs and applications, Wiley-Intersci. Publ., New York, 1985, 1-21.

[15] R. E. L. Aldred, Y. Egawa, J. Fujisawa, K. Ota and A. Saito, The existence of a 2-factor in $K_{1, n}$-free graphs with large connectivity and large edge-connectivity, (manuscript).

[16] R. E. L. Aldred, D. A. Holton and D. Lou, N-extendability of symmetric graphs, J. Graph Theory 17 (1993) (2) 253-261.

[17] R. E. L. Aldred, D. A. Holton, D. Lou and A. Saito, $M$-alternating paths in n-extendable bipartite graphs, Discrete Math. 269 (2003) (1-3) 1-11.

[18] R. E. L. Aldred, D. A. Holton, D. Lou and N. Zhong, Characterizing $2 k$-critical graphs and n-extendable graphs, Discrete Math. 287 (2004) (1-3) 135-139.

[19] R. E. L. Aldred, D. A. Holton, M. I. Porteous and M. D. Plummer, Two results on matching extensions with prescribed and proscribed edge sets, Discrete Math. 206 (1999) (1-3) 35-43.

[20] R. E. L. Aldred, D. A. Holton and J. Sheehan, 2-factors with prescribed and proscribed edges, J. Graph Theory 49 (2005) (1) 48-58.

[21] R. E. L. Aldred and B. Jackson, Edge proximity conditions for extendability in cubic bipartite graphs, J. Graph Theory 55 (2007) (2) 112-120.

[22] R. E. L. Aldred, K. Kawarabayashi and M. D. Plummer, On the matching extendability of graphs in surfaces, J. Combin. Theory Ser. B 98 (2008) (1) $105-115$.

[23] R. E. L. Aldred and M. D. Plummer, Edge proximity and matching extension in planar triangulations, Australas. J. Combin. 29 (2004) 215-224.

[24] R. E. L. Aldred and M. D. Plummer, On matching extensions with prescribed and proscribed edge sets II, Discrete Math. 197/198 (1999) 29-40.

[25] R. E. L. Aldred and M. D. Plummer, On restricted matching extension in planar graphs, Discrete Math. 231 (2001) (1-3) 73-79.

[26] N. Alon, The linear arboricity of graphs, Israel J. Math. 62 (1988) (3) 311-325.

[27] N. Alon, S. Friedland and G. Kalai, Regular subgraphs of almost regular graphs, J. Combin. Theory Ser. B 37 (1984) (1) 79-91.

[28] N. Alon and B. Sudakov, On graphs with subgraphs having large independence numbers, J. Graph Theory 56 (2007) (2) 149-157. 
[29] N. Alon, V. J. Teague and N. C. Wormald, Linear arboricity and linear $k$ arboricity of regular graphs, Graphs Combin. 17 (2001) (1) 11-16.

[30] N. Alon and R. Yuster, $H$-factors in dense graphs, J. Combin. Theory Ser. B 66 (1996) (2) 269-282.

[31] A. Amahashi, On factors with all degrees odd, Graphs Combin. 1 (1985) (1) 111-114.

[32] A. Amahashi and M. Kano, On factors with given components, Discrete Math. 42 (1982) (1) 1-6.

[33] D. Amar, E. Flandrin, G. Gancarzewicz and A. P. Wojda, Bipartite graphs with every matching in a cycle, Discrete Math. 307 (2007) (11-12) 1525-1537.

[34] N. Ananchuen, On domination critical graphs with cutvertices having connected domination number 3, Int. Math. Forum 2 (2007) (61-64) 3041-3052.

[35] N. Ananchuen, On a minimum cutset of strongly $k$-extendable graphs, J. Combin. Math. Combin. Comput. 45 (2003) 63-78.

[36] N. Ananchuen, On minimum degree of strongly $k$-extendable graphs, J. Combin. Math. Combin. Comput. 38 (2001) 149-159.

[37] N. Ananchuen, On strongly $k$-extendable graphs, J. Combin. Math. Combin. Comput. 38 (2001) 3-19.

[38] N. Ananchuen, W. Ananchuen and M. D. Plummer, Matching properties in connected domination critical graphs, Discrete Math. 308 (2008) (7) 1260-1267.

[39] N. Anunchuen and L. Caccetta, On critically k-extendable graphs, Australas. J. Combin. 6 (1992) 39-65.

[40] N. Anunchuen and L. Caccetta, On (n-2)-extendable graphs, J. Combin. Math. Combin. Comput. 16 (1994) 115-128.

[41] N. Anunchuen and L. Caccetta, On minimally k-extendable graphs, Australas. J. Combin. 9 (1994) 153-168.

[42] N. Ananchuen and L. Caccetta, A note on $k$-extendable graphs and independence number, Australas. J. Combin. 12 (1995) 59-65.

[43] N. Ananchuen and L. Caccetta, On $(n-2)$-extendable graphs. II, J. Combin. Math. Combin. Comput. 20 (1996) 65-80.

[44] N. Ananchuen and L. Caccetta, Matching extension and minimum degree, Discrete Math. 170 (1997) (1-3) 1-13. 
[45] N. Ananchuen and L. Caccetta, On strongly edge-critical graphs of given diameter, Australas. J. Combin. 8 (1993) 99-122.

[46] N. Ananchuen, L. Caccetta and W. Ananchuen, A characterization of maximal non- $k$-factor-critical graphs, Discrete Math. 307 (2007) (1) 108-114.

[47] N. Ananchuen and M. D. Plummer, 3-factor-criticality in domination critical graphs, Discrete Math. 307 (2007) (23) 3006-3015.

[48] N. Ananchuen and M. D. Plummer, Matchings in 3-vertex-critical graphs: The even case, Networks 45 (2005) (4) 210-213.

[49] N. Ananchuen and M. D. Plummer, Matchings in 3-vertex-critical graphs: The odd case, Discrete Math. 307 (2007) (13) 1651-1658.

[50] N. Ananchuen and M. D. Plummer, Matching properties in domination critical graphs, Discrete Math. 277 (2004) 1-13.

[51] N. Ananchuen and M. D. Plummer, On the connectivity and matchings in 3vertex-critical claw-free graphs, Util. Math. 69 (2006) 85-96.

[52] N. Ananchuen and M. D. Plummer, Some results related to the toughness of 3-domination critical graphs, Discrete Math. 272 (2003) (1) 5-15.

[53] N. Ananchuen and M. D. Plummer, Some results related to the toughness of 3-domination critical graphs. II, Util. Math. 70 (2006) 11-32.

[54] N. Ananchuen and A. Saito, Factor criticality and complete closure of graphs, Discrete Math. 265 (2003) (1-3) 13-21.

[55] I. Anderson, Perfect matchings of a graph, J. Combin. Theory Ser. B 10 (1971) (3) 183-186.

[56] I. Anderson, Sufficient conditions for matchings, Proc. Edinb. Math. Soc. (2) 18 (1972) 129-136.

[57] K. Ando, Y. Egawa, A. Kaneko, K. Kawarabayashi and H. Matsuda, Path factors in claw-free graphs, Discrete Math. 243 (2002) (1-3) 195-200.

[58] K. Ando and K. Kawarabayashi, Some forbidden subgraph conditions for a graph to have a $k$-contractible edge, Discrete Math. 267 (2003) (1-3) 3-11.

[59] R. P. Anstee, An algorithmic proof of Tutte's f-factor theorem, J. Algorithms 6 (1985) (1) 112-131.

[60] R. P. Anstee, Matching theory: Fractional to integral, New Zealand J. Math. 21 (1992) 17-32. 
[61] R. P. Anstee, Simplified existence theorems for $(g, f)$-factors, Discrete Appl. Math. 27 (1990) (1-2) 29-38.

[62] R. P. Anstee and L. Caccetta, Orthogonal matchings, Discrete Math. 179 (1998) (1-3) 37-47.

[63] R. P. Anstee and Y. Nam, More sufficient conditions for a graph to have factors, Discrete Math. 184 (1998) (1-3) 15-24.

[64] M. A. Babenko, On ear decompositions of strongly connected bidirected graphs, arXiv:math/0609221v1 (2006).

[65] F. Bäbler, Über die Zerlegung regulärer streckenkomplexe ungerader ordnung, Comment. Math. Helvetici 10 (1938) 275-287.

[66] B. Bai, X. Huan and Q. Yu, Every Cayley graph on dicyclic group is 2extendable (preprint).

[67] E. Balas, Integer and fractional matchings, in P. Hansen (Ed.) Annals of Discrete Mathematics (11) - Studies on Graphs and Discrete Programming, vol. 59, North-Holland, 1981, 1-13.

[68] M. L. Balinski, On maximum matching, minimum covering and their connections, in Proceedings of the Princeton Symposium on Mathematical Programming, Princeton, 1970, 303-311.

[69] M. L. Balinski, On perfect matchings, SIAM Rev. 12 (1970) (4) 570-572,

[70] M. Bartha and M. Krész, Tutte type theorems for graphs having a perfect internal matching, Inform. Process. Lett. 91 (2004) (6) 277-284.

[71] D. Bauer, H. J. Broersma, J. van den Heuvel and H. J. Veldman, On hamiltonian properties of 2-tough graphs, J. Graph Theory 18 (1994) (6) 539-543.

[72] D. Bauer, H. Broersma, N. Kahl, A. Morgana, E. Schmeichel and T. Surowiec, Tutte sets in graphs II: The complexity of finding maximum Tutte sets, Discrete Appl. Math. 155 (2007) (10) 1336-1343.

[73] D. Bauer, H. J. Broersma, A. Morgana and E. Schmeichel, Tutte sets in graphs I: Maximal Tutte sets and D-graphs, J. Graph Theory 55 (2007) (4) 343-358.

[74] D. Bauer, H. Broersma and E. Schmeichel, More progress on tough graphs the Y2K report, Electron. Notes Discrete Math. 11 (2002) 63-80.

[75] D. Bauer, H. Broersma and E. Schmeichel, Toughness in graphs - a survey, Graphs Combin. 22 (2006) (1) 1-35. 
[76] D. Bauer and E. Schmeichel, Toughness and the cycle structure of graphs, in J. W. K. John Gimbel and L. V. Quintas (Eds.) Annals of Discrete Mathematics - Quo Vadis, Graph Theory? - A Source Book for Challenges and Directions, vol. 55, Elsevier, 1993, 145-151.

[77] D. Bauer and E. Schmeichel, Toughness, minimum degree, and the existence of 2-factors, J. Graph Theory 18 (1994) (3) 241-256.

[78] C. Berge, Sur le couplage maximum d'un graphe, C. R. Acad. Sci. Paris 247(1958) 258-259.

[79] C. Berge and M. Las Vergnas, On the existence of subgraphs with degree constraints, Nederl. Akad. Wet., Proc., Ser. A 81 (1978) 165-176.

[80] J.-C. Bermond and M. Las Vergnas, Regular factors in nearly regular graphs, Discrete Math. 50 (1984) 9-13.

[81] E. Bertram and P. Horak, Decomposing 4-regular graphs into triangle-free 2factors, SIAM J. Discrete Math. 10 (1997) (2) 309-317.

[82] Q. Bian, Some results on factors and fractional factors of graphs, Ph.D. thesis, Shandong University, Shandong, China (2005).

[83] T. Biedl, E. D. Demaine, C. A. Duncan, R. Fleischer and S. G. Kobourov, Tight bounds on maximal and maximum matchings, Discrete Math. 285 (2004) (1-3) 7-15.

[84] T. Böhme, J. Harant and M. Tkáč, On certain Hamiltonian cycles in planar graphs, J. Graph Theory 32 (1999) (1) 81-96.

[85] B. Bollobás, Modern graph theory, vol. 184 of Graduate Texts in Mathematics, Springer, 1998.

[86] B. Bollobás and B. D. McKay, The number of matchings in random regular graphs and bipartite graphs, J. Combin. Theory Ser. B 41 (1986) (1) 80-91.

[87] B. Bollobás, A. Saito and N. C. Wormald, Regular factors of regular graphs, J. Graph Theory 9 (1985) (1) 97-103.

[88] J. A. Bondy, Large cycles in graphs, Discrete Math. 1 (1971) (2) 121-132.

[89] J. A. Bondy and L. Lovász, Lengths of cycles in Halin graphs, J. Graph Theory 9 (1985) (3) 397-410.

[90] J.-M. Bourjolly and W. R. Pulleyblank, König-Egerváry graphs, 2-bicritical graphs and fractional matchings, Discrete Appl. Math. 24 (1989) (1-3) 63-82. 
[91] S. Brandt, Private communication.

[92] S. Brandt, G. Chen, R. Faudree, R. J. Gould and L. Lesniak, Degree conditions for 2-factors, J. Graph Theory 24 (1997) (2) 165-173.

[93] R. C. Brigham, P. Z. Chinn and R. D. Dutton, Vertex domination-critical graphs, Networks 18 (1988) (3) 173-179.

[94] R. C. Brigham, T. W. Haynes, M. A. Henning and D. F. Rall, Bicritical domination, Discrete Math. 305 (2005) (1-3) 18-32.

[95] H. J. Broersma, M. Kriesell and Z. Ryjáček, On factors of 4-connected claw-free graphs, J. Graph Theory 37 (2001) (2) 125-136.

[96] H. J. Broersma, Z. Ryjáček and I. Schiermeyer, Dirac's minimum degree condition restricted to claws, Discrete Math. 167-168 (1997) 155-166.

[97] J. Brousek, Forbidden triples for hamiltonicity, Discrete Math. 251 (2002) (1-3) $71-76$.

[98] J. Brousek, Z. Ryjáček and O. Favaron, Forbidden subgraphs, hamiltonicity and closure in claw-free graphs, Discrete Math. 196 (1999) (1-3) 29-50.

[99] J. Brousek, Z. Ryjáček and I. Schiermeyer, Forbidden subgraphs, stability and hamiltonicity, Discrete Math. 197-198 (1999) 143-155.

[100] H. L. I. Buchanan, Matchings including or excluding certain edge sets in bipartite graphs, Bull. London Math. Soc. 29 (1997) (3) 303-306.

[101] J. Cai and G. Liu, Stability number and $f$-factors in $K_{1, n}$-free graphs, Ars Combin. (to appear).

[102] J. Cai and G. Liu, Stability number and fractional $f$-factors in graphs, Ars Combin. 80 (2006) 141-146.

[103] M. Cai, [a,b]-factorizations of graphs, J. Graph Theory 15 (1991) (3) 283-301.

[104] M. Cai, A degree condition for the existence of connected $[k, k+1]$-factors, $J$. Syst. Sci. Complex. 8 (1995) (4) 364-368.

[105] M. Cai, Connected [k,k+1]-factors of graphs, Discrete Math. 169 (1997) (1-3) $1-16$.

[106] M. Cai, On some factor theorems of graphs, Discrete Math. 98 (1991) (3) 223229.

[107] M. Cai, O. Favaron and H. Li, (2,k)-factor-critical graphs and toughness, Graphs Combin. 15 (1999) (2) 137-142. 
[108] M. Cai and Y. Li, A $[k, k+1]$-factor containing a given Hamiltonian cycle, Electron. J. Combin. 6 (1999) (1) R4.

[109] M. Cai, Y. Li and M. Kano, A $[k, k+1]$-factor containing a given hamiltonian cycle, Science in China Series A 41 (1998) (9) 933-938.

[110] K. Cameron, Coloured matchings in bipartite graphs, Discrete Math. 169 (1997) (1-3) 205-209.

[111] K. Cameron and T. Walker, The graphs with maximum induced matching and maximum matching the same size, Discrete Math. 299 (2005) (1-3) 49-55.

[112] C. N. Campos and C. L. Lucchesi, On the relation between the Petersen graph and the characteristic of separating cuts of matching covered graphs, Institute of Computing, Unicamp, (2000).

[113] Y. Caro, On induced subgraphs with odd degrees, Discrete Math. 132 (1994) (13) 23-28.

[114] M. H. de Carvalho, An $O(V E)$ algorithm for ear decompositions of matchingcovered graphs, ACM Trans. Algorithms 1 (2005) (2) 324-337.

[115] M. H. de Carvalho and C. H. C. Little, Ear decompositions in combed graphs, Electron. J. Combin. 15 (2008) (1) R19.

[116] M. H. de Carvalho and C. H. C. Little, Minimal bipartite join covered graphs, to appear.

[117] M. H. de Carvalho and C. L. Lucchesi, Matching covered graphs and subdivisions of $K_{4}$ and $\bar{C}_{6}, J$. Combin. Theory Ser. B 66 (1996) (2) 263-268.

[118] M. H. Carvalho, C. L. Lucchesi and U. S. R. Murty, Ear decompositions of matching covered graphs, Combinatorica 19 (1999) (2) 151-174.

[119] M. H. de Carvalho, C. L. Lucchesi and U. S. R. Murty, How to build a brace, Institute of Computing, University of Campinas, (2005).

[120] M. H. de Carvalho, C. L. Lucchesi and U. S. R. Murty, On a conjecture of Lovász concerning bricks, Institute of Computing, University of Campinas, (1998).

[121] M. H. de Carvalho, C. L. Lucchesi and U. S. R. Murty, On a conjecture of Lovász concerning bricks: I. the characteristic of a matching covered graph, $J$. Combin. Theory Ser. B 85 (2002) (1) 94-136.

[122] M. H. de Carvalho, C. L. Lucchesi and U. S. R. Murty, On a conjecture of Lovász concerning bricks: II. bricks of finite characteristic, J. Combin. Theory Ser. B 85 (2002) (1) 137-180. 
[123] M. H. de Carvalho, C. L. Lucchesi and U. S. R. Murty, Optimal ear decompositions of matching covered graphs and bases for the matching lattice, J. Combin. Theory Ser. B 85 (2002) (1) 59-93.

[124] M. H. de Carvalho, C. L. Lucchesi and U. S. R. Murty, The matching lattice, in B. A. Reed (Ed.) Recent advances in algorithms and combinatorics, Springer, New York, 2003, 1-25.

[125] M. H. de Carvalho, C. L. Lucchesi and U. S. R. Murty, The perfect matching polytope and solid bricks, J. Combin. Theory Ser. B 92 (2004) (2) 319-324.

[126] M. H. de Carvalho, C. L. Lucchesi and U. S. R. Murty, Graphs with independent perfect matchings, J. Graph Theory 48 (2005) (1) 19-50.

[127] M. H. de Carvalho, C. L. Lucchesi and U. S. R. Murty, How to build a brick, Discrete Math. 306 (2006) (19-20) 2383-2410.

[128] F. Castagna and G. Prins, Every generalized Petersen graph has a Tait coloring, Pacific Journal of Mathematics 40 (1972) 53-58.

[129] P. A. Catlin, Subgraphs of graphs, I, Discrete Math. 10 (1974) (2) 225-233.

[130] P. A. Catlin and H.-J. Lai, Supereulerian graphs and the Petersen graph, J. Combin. Theory Ser. B 66 (1996) (1) 123-139.

[131] C. Chan and T. Nishimura, A recursive theorem on matching extension, Australas. J. Combin. 21 (2000) 49-55.

[132] O. Chan, C. C. Chen and Q. Yu, On 2-extendable abelian Cayley graphs, Discrete Math. 146 (1995) (1-3) 19-32.

[133] G. J. Chang, C. Lu, J. Wu and Q. Yu, Vertex coloring 2-edge weighting of bipartite graphs, (manuscript) 2007.

[134] C. Chen, Binding number and minimum degree for $[a, b]$-factors, Syst. Sci. Math. Sci. 6 (1993) (2) 179-185.

[135] C. Chen, Binding number and toughness for matching extension, Discrete Math. 146 (1995) (1-3) 303-306.

[136] C. Chen, Matchings and matching extensions in graphs, Discrete Math. 186 (1998) (1-3) 95-103.

[137] C. Chen, Minimum degree and binding number for $k$-factors with prescribed properties, Acta Math. Sci. Ser. B Engl. Ed. 12 (1992) (4) 374-380.

[138] C. Chen, On matchings and $k$-factors in graphs with odd-cycle property, Congr. Numer. 109 (1995) 135-140. 
[139] C. Chen, Remarks on the binding number for $[a, b]$-factors, Syst. Sci. Math. Sci. 5 (1992) (2) 141-145.

[140] C. Chen, Remarks on graphs with odd-cycle property, Util. Math. 45 (1994) 229-236.

[141] C. Chen, Toughness of graphs and $k$-factors with given properties, Ars Combin. 34 (1992) 55-64.

[142] C. Chen, Toughness of graphs and [2,b]-factors, Graphs Combin. 10 (1994) (2) 97-100.

[143] C. Chen and G. Liu, Toughness of graphs and $[a, b]$-factors with prescribed properties, J. Combin. Math. Combin. Comput. 12 (1992) 215-221.

[144] C. Chen and J. Wang, Factors in graphs with odd-cycle property, Discrete Math. 112 (1993) (1-3) 29-40.

[145] C. C. Chen, J. Liu and Q. Yu, On the classification of 2-extendable Cayley graphs on dihedral groups, Australas. J. Combin. 6 (1992) 209-219.

[146] C. C. Chen and N. F. Quimpo, On strongly hamiltonian abelian group graphs, Combinatorial mathematics VIII, Proc. 8th Aust. Conf., Geelong/Aust. 1980, Lect. Notes Math. 884, 23-34. (1981).

[147] G. Chen, J. R. Faudree, R. J. Gould and A. Saito, 2-factors in claw-free graphs, Discuss. Math. Graph Theory 20 (2000) (2) 165-172.

[148] G. Chen, R. J. Faudree, R. J. Gould, M. S. Jacobson, L. Lesniak and F. Pfender, Linear forests and ordered cycles, Discuss. Math. Graph Theory 24 (2004) (3) 359-372.

[149] G. Chen, R. J. Gould and M. S. Jacobson, On 2-factors containing 1-factors in bipartite graphs, Discrete Math. 197-198 (1999) 185-194.

[150] Y. Chen, F. Tian and B. Wei, Degree sums and path-factors in graphs, Graphs Combin. 17 (2001) (1) 61-71.

[151] S. A. Choudum and M. S. Paulraj, Regular factors in $K_{1,3}$-free graphs, J. Graph Theory 15 (1991) (3) 259-265.

[152] V. Chungphaisan, Factors of graphs and degree sequences, Nanta Math. 9 (1976) 41-49.

[153] V. Chvátal, Tough graphs and Hamiltonian circuits, Discrete Math. 5 (1973) (3) 215-228. 
[154] V. Chvátal and P. Erdös, A note on Hamiltonian circuits, Discrete Math. 2 (1972) (2) 111-113.

[155] V. Chvátal, H. Fleischner, J. Sheehan and C. Thomassen, Three-regular subgraphs of four-regular graphs, J. Graph Theory 3 (1979) (4) 371-386.

[156] M. Ciucu, Enumeration of perfect matchings in graphs with reflective symmetry, J. Combin. Theory Ser. A 77 (1997) (1) 67-97.

[157] G. Cornuéjols, General factors of graphs, J. Combin. Theory Ser. $B 45$ (1988) (2) 185-198.

[158] G. Cornuéjols and W. Pulleyblank, A matching problem with side conditions, Discrete Math. 29 (1980) (2) 135-159.

[159] J. R. Correa and M. Matamala, Some remarks about factors of graphs, J. Graph Theory 57 (2008) (4) 265-274.

[160] Y. Cui and M. Kano, Some results on odd factors of graphs, J. Graph Theory 12 (1988) (3) 327-333.

[161] W. H. Cunningham, Matching, matroids, and extensions, Math. Program. 91 (2002) (3) 515-542.

[162] N. Dean, The matching extendability of surfaces, J. Combin. Theory Ser. B 54 (1992) (1) 133-141.

[163] N. Dean and J. Zito, Well-covered graphs and extendability, Discrete Math. 126 (1994) (1-3) 67-80.

[164] G. A. Dirac, Some theorems on abstract graphs, Proc. London Math. Soc. (3) 2 (1952) 69-81.

[165] J. Donald, J. Elwin, R. Hager and P. Salamon, Handle bases and bounds on the number of subgraphs, J. Combin. Theory, Ser. B 42 (1987) 1-13.

[166] T. Došlić, Counting perfect matchings in $n$-extendable graphs, Discrete Math. 308 (2008) (11) 2297-2300.

[167] T. Došlić, On some structural properties of Fullerene graphs, J. Math. Chem. 31 (2002) (2) 187-195.

[168] J. Edmonds, Paths, trees, and flowers, Canad. J. Math. 17 (1965) 449-467.

[169] J. Edmonds, Matroids and the greedy algorithm, Math. Program. 1 (1971) (1) $127-136$. 
[170] J. Edmonds, W. Pulleyblank and L. Lovász, Brick decompositions and the matching rank of graphs, Combinatorica 2 (1982) (3) 247-274.

[171] Y. Egawa, Era's conjecture on $[k, k+1]$-factorizations of regular graphs, Ars Combin. 21 (1986) 217-220.

[172] Y. Egawa and H. Enomoto, Sufficient conditions for the existence of $k$-factors, in Recent studies in graph theory, Vishwa international Publications, 1989, 96105.

[173] Y. Egawa, H. Enomoto, R. J. Faudree, H. Li and I. Schiermeyer, Two-factors each component of which contains a specified vertex, J. Graph Theory 43 (2003) (3) 188-198.

[174] Y. Egawa, H. Enomoto and A. Saito, Factors and induced subgraphs, Discrete Math. 68 (1988) (2-3) 179-189.

[175] Y. Egawa, H. Enomoto and A. Saito, On component factors, Graphs Combin. 2 (1986) (1) 223-225.

[176] Y. Egawa, R. J. Faudree, E. Györi, Y. Ishigami, R. H. Schelp and H. Wang, Vertex-disjoint cycles containing specified edges, Graphs Combin. 16 (2000) (1) 81-92.

[177] Y. Egawa, S. Fujita and K. Ota, $K_{1,3}$-factors in graphs, Discrete Math. In Press, Corrected Proof -.

[178] Y. Egawa and M. Kano, Sufficient conditions for graphs to have $(g, f)$-factors, Discrete Math. 151 (1996) (1-3) 87-90.

[179] Y. Egawa, M. Kano and A. K. Kelmans, Star partitions of graphs, J. Graph Theory 25 (1997) (3) 185-190.

[180] Y. Egawa and K. Ota, Regular factors in $K_{1, n}$-free graphs, J. Graph Theory 15 (1991) (3) 337-344.

[181] Y. Egawa and K. Ota, Vertex-disjoint claws in graphs, Discrete Math. 197-198 (1999) 225-246.

[182] E. Egerváry, On combinatorial properties of matrices. Math. Lapok 38 (1931) $16-28$.

[183] M. H. El-Zahar, On circuits in graphs, Discrete Math. 50 (1984) 227-230.

[184] M. N. Ellingham, Y. Nam and H.-J. Voss, Connected $(g, f)$-factors, J. Graph Theory 39 (2002) (1) 62-75. 
[185] M. N. Ellingham and X. Zha, Toughness, trees, and walks, J. Graph Theory 33 (2000) (3) 125-137.

[186] H. Enomoto, Toughness and the existence of $k$-factors. II, Graphs Combin. 2 (1986) (1) 37-42.

[187] H. Enomoto, Toughness and the existence of $k$-factors. III, Discrete Math. 189 (1998) (1-3) 277-282.

[188] H. Enomoto and M. Hagita, Toughness and the existence of $k$-factors. IV, Discrete Math. 216 (2000) (1-3) 111-120.

[189] H. Enomoto, B. Jackson, P. Katerinis and A. Saito, Toughness and the existence of $k$-factors, J. Graph Theory 9 (1985) (1) 87-95.

[190] H. Enomoto, A. Kaneko and Z. Tuza, $P_{3}$-factors and covering cycles in graphs of minimum degree $n / 3$, in Combinatorics, Proc. 7th Hung. Colloq., Eger/Hung, vol. 52 of Colloq. Math. Soc. János Bolyai, Colloq. Math. Soc. János Bolyai, 1988, 213-220.

[191] H. Enomoto and S. Matsunaga, Graph decompositions without isolated vertices. II, J. Math. Soc. Japan 49 (1997) (1) 161-180.

[192] H. Enomoto and S. Matsunaga, Graph decompositions without isolated vertices III, J. Graph Theory 24 (1997) (2) 155-164.

[193] H. Enomoto, K. Ota and M. Kano, A sufficient condition for a bipartite graph to have a $k$-factor, J. Graph Theory 12 (1988) (1) 141-151.

[194] H. Enomoto, M. D. Plummer and A. Saito, Neighborhood unions and factor critical graphs, Discrete Math. 205 (1999) (1-3) 217-220.

[195] H. Enomoto and T. Tokuda, Complete-factors and f-factors, Discrete Math. 220 (2000) (1-3) 239-242.

[196] G.-H. Fan, New sufficient conditions for cycles in graphs, J. Combin. Theory Ser. B 37 (1984) (3) 221-227.

[197] R. J. Faudree, Survey of results on k-ordered graphs, Discrete Math. 229 (2001) (1-3) 73-87.

[198] J. R. Faudree, R. J. Faudree, R. J. Gould, M. S. Jacobson, L. Lesniak, On k-ordered graphs, J. Graph Theory 35 (2000) 69-82.

[199] J. R. Faudree, R. J. Faudree and Z. Ryjáček, Forbidden subgraphs that imply 2-factors, Discrete Math. 308 (2008) (9) 1571-1582. 
[200] R. J. Faudree, O. Favaron, E. Flandrin, H. Li and Z. Liu, On 2-factors in claw-free graphs, Discrete Math. 206 (1999) (1-3) 131-137.

[201] R. J. Faudree, E. Flandrin and Z. Ryjáček, Claw-free graphs - a survey, Discrete Math. 164 (1997) (1-3) 87-147.

[202] R. J. Faudree, R. J. Gould, M. S. Jacobson, L. Lesniak and A. Saito, A note on 2-factors with two components, Discrete Math. 300 (2005) (1-3) 218-224.

[203] R. J. Faudree, R. J. Gould, M. S. Jacobson, L. Lesniak and A. Saito, Toughness, degrees and 2-factors, Discrete Math. 286 (2004) (3) 245-249.

[204] R. J. Faudree, R. J. Gould, A. V. Kostochka, L. Lesniak, I. Schiermeyer and A. Saito, Degree conditions for $k$-ordered hamiltonian graphs, J. Graph Theory 42 (2003) (3) 199-210.

[205] O. Favaron, Extendability and factor-criticality, Discrete Math. 213 (2000) (1-3) 115-122.

[206] O. Favaron, On $k$-factor-critical graphs, Discuss. Math. Graph Theory 16 (1996) (1) 41-51.

[207] O. Favaron, E. Flandrin and Z. Ryjáček, Factor-criticality and matching extension in DCT-graphs, Discuss. Math. Graph Theory 17 (1997) (2) 271-278.

[208] O. Favaron, M. C. Heydemann, J. C. Meyer and D. Sotteau, A parameter linked with $G$-factors and the binding number, Discrete Math. 91 (1990) (3) 311-316.

[209] O. Favaron and M. Shi, Minimally $k$-factor-critical graphs, Australas. J. Combin. 17 (1998) 89-97.

[210] O. Favaron, F. Tian and L. Zhang, Independence and hamiltonicity in 3domination-critical graphs, J. Graph Theory 25 (1997) (3) 173-184.

[211] H. Feng and G. Liu, Orthogonal factorizations of graphs, J. Graph Theory 40 (2002) (4) 267-276.

[212] J. Fink, Perfect matchings extend to Hamilton cycles in hypercubes, J. Combin. Theory Ser. B 97 (2007) (6) 1074-1076.

[213] I. Fischer and C. H. C. Little, A characterisation of Pfaffian near bipartite graphs, J. Combin. Theory Ser. B 82 (2001) (2) 175-222.

[214] E. Flandrin, F. Tian, B. Wei and L. Zhang, Some properties of 3-dominationcritical graphs, Discrete Math. 205 (1999) 65-76.

[215] J. Folkman and D. R. Fulkerson, Flows in infinite graphs, Journal of Combinatorial Theory 8 (1970) (1) 30-44. 
[216] P. Fraisse, P. Hell and D. G. Kirkpatrick, A note on $f$-factors in directed and undirected multigraphs, Graphs Combin. 2 (1986) (1) 61-66.

[217] A. Frank, Conservative weightings and ear-decompositions of graphs, Combinatorica 13 (1993) (1) 65-81.

[218] A. Frank, L. Chi Lau and J. Szabó, A note on degree-constrained subgraphs, Discrete Math. 308 (2008) (12) 2647-2648.

[219] A. Frank, E. Györi and A. Sebü, A question on matching extendability, London Math. Soc., Lecture Notes, Vol. 141 (1989), 84-114.

[220] S. Fujita, K. Kawarabayashi, C. L. Lucchesi, K. Ota, M. D. Plummer and A. Saito, A pair of forbidden subgraphs and perfect matchings, J. Combin. Theory Ser. B 96 (2006) (3) 315-324.

[221] D. Fulkerson, A. Hoffman and M. McAndrew, Some properties of graphs with multiple edges, Canad. J. Math. 17 (1965) 166-177.

[222] J. Fulman, Domination in vertex and edge critical graphs, (manuscript) 1992.

[223] J. Fulman, D. Hanson and G. Macgillivray, Vertex domination-critical graphs, Networks 25 (1995) (2) 41-43.

[224] H. N. Gabow, Algorithmic proofs of two relations between connectivity and the 1-factors of a graph, Discrete Math. 26 (1979) (1) 33-40.

[225] H. N. Gabow, H. Kaplan and R. E. Tarjan, Unique maximum matching algorithms, J. Algorithms 40 (2001) (2) 159-183.

[226] T. Gallai, On factorisation of graphs, Acta Math. Hungar. 1 (1950) (1) 133-153.

[227] G. Gancarzewicz and A. P. Wojda, Graphs with every $k$-matching in a Hamiltonian cycle, Discrete Math. 213 (2000) (1-3) 141-151.

[228] M. R. Garey and D. S. Johnson, Computers and Intractability: A Guide to the Theory of NP-completeness, W. H. Freeman and Company, Publishers, San Francisco, 1979.

[229] C. Godsil and G. Royle, Algebraic graph theory, vol. 207 of Graduate Texts in Mathematics, New York, NY: Springer, 2001.

[230] F. Göring and G. Katona, Local topological toughness and local factors, Graphs Combin. 23 (2007) (4) 387-399.

[231] R. J. Gould, Advances on the Hamiltonian problem - a survey, Graphs Combin. 19 (2003) (1) 7-52. 
[232] R. J. Gould, Updating the hamiltonian problem - survey, J. Graph Theory 15 (1991) (2) 121-157.

[233] R. J. Gould and J. M. Harris, Traceability in graphs with forbidden triples of subgraphs, Discrete Math. 189 (1998) (1-3) 123-132.

[234] F. Guldan, Some results on linear arboricity, J. Graph Theory 10 (1986) 505509.

[235] P. Gvozdjak and J. Nešetřil, High girth and extendability, Discrete Math. 154 (1996) (1-3) 77-84.

[236] E. Györi and W. Imrich, On the strong product of a $k$-extendable and an $l$ extendable graph, Graphs Combin. 17 (2001) (2) 245-253.

[237] E. Györi and M. D. Plummer, The cartesian product of a $k$-extendable and an $l$-extendable graph is $(k+l+1)$-extendable, Discrete Math. 101 (1992) (1-3) 87-96.

[238] M. Habib and B. Peroche, Some problems about linear arboricity, Discrete Math. 41 (1982) (2) 219-220.

[239] J. Haglund and J. B. Remmel, Cycles and perfect matchings, Discrete Math. 274 (2004) (1-3) 93-108.

[240] A. Hajnal and E. Szemerédi, Proof of a conjecture of Erdös, Comninatorial Theorey and its Applications, Vol. II, Eds. Erodös, Rényi and Sós, Colloq. Math. Soc. J. Bolyai 4, North-Holland, Amersterdam, 1970, 601-623.

[241] P. Hansen, F. Zhang and M. Zheng, Perfect matchings and ears in elementary bipartite graphs, Discrete Math. 176 (1997) (1-3) 131-138.

[242] K. Heinrich, P. Hell, D. G. Kirkpatrick and G. Liu, A simple existence criterion for $(g<f)$ - factors, Discrete Math. 85 (1990) (3) 313-317.

[243] K. Heinrich, P. Horak, W. Wallis and Q. Yu, Perfect double covers with paths of length four, J. Graph Theory 21 (1996) (2) 187-197.

[244] G. Helde and O. Vieten, Hamilton cycles in maximal planar graphs, Electron. Notes Discrete Math. 25 (2006) 71-71.

[245] P. Hell, Graph packings, Electron. Notes Discrete Math. 5 (2000) 170-173.

[246] P. Hell and D. G. Kirkpatrick, Packings by cliques and by finite families of graphs, Discrete Math. 49 (1984) (1) 45-59.

[247] G. R. T. Hendry, Maximum graphs with a unique $k$-factor, J. Combin. Theory Ser. B 37 (1984) (1) 53-63. 
[248] A. J. W. Hilton, Factorizations of regular graphs of high degree, J. Graph Theory 9 (1985) (1) 193-196.

[249] A. Hoffmann, Regular factors in regular multipartite graphs, Discrete Math. 258 (2002) (1-3) 43-62.

[250] A. Hoffmann, E. Sidorowicz and L. Volkmann, Extremal bipartite graphs with a unique $k$-factor, Discuss. Math. Graph Theory 26 (2006) 181-192.

[251] A. Hoffmann and L. Volkmann, On unique $k$-factors and unique $[1, k]$-factors in graphs, Discrete Math. 278 (2004) (1-3) 127-138.

[252] D. A. Holton and D. Lou, Matching extension of strongly regular graphs, Australas. J. Combin. 6 (1992) 187-208.

[253] D. A. Holton and D. Lou, N-extendability of line graphs, (preprint).

[254] D. A. Holton, D. Lou and K. L. McAvaney, n-extendability of line graphs, power graphs, and total graphs, Australas. J. Combin. 11 (1995) 215-222.

[255] D. A. Holton, D. Lou and M. D. Plummer, On the 2-extendability of planar graphs, Discrete Math. 96 (1991) (2) 81-99.

[256] D. A. Holton and M. D. Plummer, 2-extendability in 3-polytopes, in Combinatorics, Proc. 7th Hung. Colloq., Eger/Hung., vol. 52 of Colloq. Math. Soc. Jnos Bolyai, 1988, 281-300.

[257] D. A. Holton and M. D. Plummer, Cycles through prescribed and forbidden points sets, Ann. Dicrete Math. 16 (1982) 129-147.

[258] R. Hu and D. Lou, Two Theorems of Matchings, Discrete Math. (to appear).

[259] Z. Hu, A generalization of Fan's condition and forbidden subgraph conditions for hamiltonicity, Discrete Math. 196 (1999) (1-3) 167-175.

[260] T. Iida and T. Nishimura, An Ore-type condition for the existence of $k$-factors in graphs, Graphs Combin. 7 (1991) (4) 353-361.

[261] T. Iida and T. Nishimura, Neighborhood conditions and $k$-factors, Tokyo J. Math. 20 (1997) (2) 411-418.

[262] Y. Ishigami and T. Jiang, Vertex-disjoint cycles containing prescribed vertices, J. Graph Theory 42 (2003) (4) 276-296.

[263] Y. Ishigami and H. Wang, An extension of a theorem on cycles containing specified independent edges, Discrete Math. 245 (2002) (1-3) 127-137. 
[264] B. Jackson and O. Ordaz, A chvátal-erdös condition for (1,1)-factors in digraphs, Discrete Math. 57 (1985) (1-2) 199-201.

[265] B. Jackson and R. W. Whitty, A note concerning graphs with unique $f$-factors, J. Graph Theory 13 (1989) (5) 577-580.

[266] K. Jacobs and D. Heiratssatz, in Selecta Mathematica 1, Springer-Verlag, Heidelberg, 1969, 103-141.

[267] S. Janson, T. Łuczak and A. Ruciński, Random graphs, Wiley-Interscience Series in Discrete Mathematics and Optimization, New York, 2000.

[268] Z. Jin, H. Yan and Q. Yu, Generalization of matching extensions in graphs (II), Discrete Appl. Math. 155 (2007) (10) 1267-1274.

[269] A. Joentgen and L. Volkmann, Factors of locally almost regular graphs, Bull. London Math. Soc. 23 (1991) (2) 121-122.

[270] P. Johann, On the structure of graphs with a unique $k$-factor, J. Graph Theory 35 (2000) (4) 227-243.

[271] R. Johansson, An El-Zahár type condition ensuring path-factors, J. Graph Theory 28 (1998) (1) 39-42.

[272] A. Kaneko, A necessary and sufficient condition for the existence of a path factor every component of which is a path of length at least two, J. Combin. Theory Ser. B 88 (2003) (2) 195-218.

[273] A. Kaneko, Paths and cycles concerning independent edges, Graphs Combin. 6 (1990) (4) 339-363.

[274] A. Kaneko and K. Ota, On minimally $(n, \lambda)$-connected graphs, J. Combin. Theory Ser. B 80 (2000) (1) 156-171.

[275] A. Kaneko and K. Yoshimoto, A 2-factor with two components of a graph satisfying the Chvátal-Erdös condition, J. Graph Theory 43 (2003) (4) 269279.

[276] A. Kaneko and K. Yoshimoto, On a 2-factor with a specified edge in a graph satisfying the Ore condition, Discrete Math. 257 (2002) (2-3) 445-461.

[277] M. Kano, [a,b]-factorization of a graph, J. Graph Theory 9 (1985) (1) 129-146.

[278] M. Kano, A sufficient condition for a graph to have $[a, b]$-factors, Graphs Combin. 6 (1990) (3) 245-251.

[279] M. Kano, An Ore-type sufficient condition for a gaph to have a connected $[2, k]$ factor, private communication. 
[280] M. Kano, Factors of regular graphs, J. Combin. Theory Ser. B 41 (1986) (1) $27-36$.

[281] M. Kano, Graph factors with given properties, in Graph theory, Proc. 1st Southeast Asian Colloq., Singapore 1983, Lect. Notes Math. 1073, 1984, 161-168.

[282] M. Kano, Regular subgraphs of a regular graph, Graph theory and its applications: East and West (Jinan, 1986), Ann. New York Acad. Sci., 576, New York Acad. Sci., New York (1989), 281-284.

[283] M. Kano, Some current results and problems on factors of graphs, in: Proc. 3rd China-USA Internat. Conf. on Graph Theory and its Applications (World Sci. Publishing, River Edge, New York, 1994) 93-98.

[284] M. Kano, Sufficient conditions for a graph to have factors, Discrete Math. 80 (1990) (2) 159-165.

[285] M. Kano and G. Y. Katona, Odd subgraphs and matchings, Discrete Math. 250 (2002) (1-3) 265-272.

[286] M. Kano and G. Y. Katona, Structure theorem and algorithm on $(1, f)$-odd subgraph, Discrete Math. 307 (2007) (11-12) 1404-1417.

[287] M. Kano, C. Lee and K. Suzuki, Path and cycle factors of cubic bipartite graphs, (manuscript).

[288] M. Kano and H. Matsuda, Some results on $(1, f)$-odd factors, in Combinatorics, graph theory, and algorithms, New Issues Press, Kalamazoo, MI, 1999, 527-533.

[289] M. Kano and A. Saito, [a,b]-factors of graphs, Discrete Math. 47 (1983) 113116.

[290] M. Kano and N. Tokushige, Binding numbers and $f$-factors of graphs, J. Combin. Theory Ser. B 54 (1992) (2) 213-221.

[291] M. Kano and Q. Yu, Pan-factorial property in regular graphs, Electron. J. Combin. 12 (2005) (1) N23.

[292] M. Karoński, T. Łuczak and A. Thomason, Edge weights and vertex colours, J. Combin. Theory Ser. B 91 (2004) (1) 151-157.

[293] P. Katerinis, A Chvátal-Erdös condition for an $r$-factor in a graph, Ars Combin. 20 (1985) 185-191.

[294] P. Katerinis, Minimum degree of bipartite graphs and the existence of $k$-factors, Graphs Combin. 6 (1990) (3) 253-258. 
[295] P. Katerinis, Regular factors in regular graphs, Discrete Math. 113 (1993) (1-3) 269-274.

[296] P. Katerinis, Some conditions for the existence of $f$-factors, J. Graph Theory 9 (1985) (4) 513-521.

[297] P. Katerinis, Some results on the existence of $2 n$-factors in terms of vertexdeleted subgraphs, Ars Combin. 16 (1983) 271-277.

[298] P. Katerinis, Toughness of graphs and 2-factors with given properties, Ars Combin. (to appear).

[299] P. Katerinis, Toughness of graphs and the existence of factors, Discrete Math. 80 (1990) (1) 81-92.

[300] P. Katerinis, Two sufficient conditions for a 2-factor in a bipartite graph, J. Graph Theory 11 (1987) (1) 1-6.

[301] P. Katerinis and N. Tsikopoulos, Independence number, connectivity and $f$ factors, Util. Math. 57 (2000) 81-95.

[302] P. Katerinis and N. Tsikopoulos, Minimum degree and $f$-factors in graphs, New Zealand J. Math. 29 (2000) (1) 33-40.

[303] P. Katerinis and N. Tsikopoulos, Perfect matchings in regular bipartite graphs, Graphs Combin. 12 (1996) (1) 327-331.

[304] P. Katerinis and D. R. Woodall, Binding numbers of graphs and the existence of k-factors, Q. J. Math., Oxf. II. Ser. 38 (1987) (2) 221-228.

[305] G. Y. Katona, Properties of edge-tough graphs, Graphs Combin. 15 (1999) (3) 315-325.

[306] G. Y. Katona, Toughness and edge-toughness, Discrete Math. 164 (1997) (1-3) 187-196.

[307] K. Kawarabayashi, $K_{4}^{-}$-factor in a graph, J. Graph Theory 39 (2002) 111-128.

[308] K. Kawarabayashi, A theorem on paths in locally planar triangulations, European J. Combin. 25 (2004) (6) 781-784.

[309] K. Kawarabayashi, H. Matsuda, Y. Oda and K. Ota, Path factors in cubic graphs, J. Graph Theory 39 (2002) (3) 188-193.

[310] K. Kawarabayashi, K. Ota and A. Saito, Hamiltonian cycles in $n$-factor-critical graphs, Discrete Math. 240 (2001) (1-3) 71-82. 
[311] K. Kawarabayashi, K. Ota and A. Saito, Hamiltonian cycles in $n$-extendable graphs, J. Graph Theory 40 (2002) (2) 75-82.

[312] K. Kawarabayashi, M. D. Plummer and A. Saito, Domination in a graph with a 2-factor, J. Graph Theory 52 (2006) (1) 1-6.

[313] K. Kawarabayashi, M. D. Plummer and A. Saito, On two equimatchable graph classes, Discrete Math. 266 (2003) (1-3) 263-274.

[314] A. K. Kelmans, Optimal packing of induced stars in a graph, Discrete Math. 173 (1997) (1-3) 97-127.

[315] C. Kenyon and E. Rémila, Perfect matchings in the triangular lattice, Discrete Math. 152 (1996) (1-3) 191-210.

[316] H. A. Kierstead, An Effective Version of Hall's Theorem, Proc. of Ameri. Math. Soc. 88 (1983) 124-128.

[317] K. Kimura, Factors and vertex-deleted subgraphs, Discrete Math. 306 (2006) (6) 607-611.

[318] Z. Király and J. Szabó, Induced Factor Problems, (manuscript).

[319] D. G. Kirkpatrick and P. Hell, On the complexity of general graph factor problems, SIAM J. Comput. 12 (1983) (3) 601-609.

[320] S. Kleinerman, Bounds on the forcing numbers of bipartite graphs, Discrete Math. 306 (2006) (1) 66-73.

[321] K. Kotani, Factors and connected induced subgraphs, Graphs Combin. 17 (2001) (3) 511-515.

[322] A. Kotlov, Matchings and Hadwiger's conjecture, Discrete Math. 244 (2002) (13) $241-252$.

[323] A. Kotlov, Short Proof of the Gallai-Edmonds Structure Theorem, (unpublished) arXiv:math.Co/0011204 v1, Nov. 2000.

[324] M. Kouider, Covering vertices by cycles, J. Graph Theory 18 (1994) (8) 757-776.

[325] M. Kouider and Z. Lonc, Stability number and $[a, b]$-factors in graphs, J. Graph Theory 46 (2004) (4) 254-264.

[326] M. Kouider and M. Mahéo, 2-edge-connected [2, k]-factors in graphs, J. Combin. Math. Combin. Comput. 35 (2000) 89-95.

[327] M. Kouider and M. Mahéo, Connected [a,b]-factors in graphs, Combinatorica 22 (2002) (1) 71-82. 
[328] M. Kouider and G. Sabidussi, Factorizations of 4-regular graphs and Petersen's theorem, J. Combin. Theory Ser. B 63 (1995) (2) 170-184.

[329] M. Kouider and D. Sotteau, On the existence of a matching orthogonal to a 2-factorization, Discrete Math. 73 (1989) (3) 301-304.

[330] M. Kouider and P. D. Vestergaard, Connected factors in graphs - a survey, Graphs Combin. 21 (2005) (1) 1-26.

[331] S. Kundu, Generalizations of the k-factor theorem, Discrete Math. 9 (1974) (2) 173-179.

[332] S. Kundu, The $k$-factor conjecture is true, Discrete Math. 6 (1973) (4) 367-376.

[333] D. Labbate, Characterizing minimally 1-factorable $r$-regular bipartite graphs, Discrete Math. 248 (2002) (1-3) 109-123.

[334] F. Lam and L. Pachter, Forcing numbers of stop signs, Theoretical Computer Science 303 (2003) (2-3) 409-416.

[335] P. C. B. Lam, G. Liu, G. Li and W. C. Shiu, Orthogonal ( $g, f)$-factorizations in networks, Networks 35 (2000) (4) 274-278.

[336] M. Las Vergnas, An extension of Tutte's 1-factor theorem, Discrete Math. 23 (1978) (3) 241-255.

[337] U. Lenkewitz and L. Volkmann, Neighbourhood and degree conditions for the existence of regular factors, Ars Combin. 42 (1996) 33-47.

[338] J. Li, A new degree condition for graphs to have $[a, b]$-factor, Discrete Math. 290 (2005) (1) 99-103.

[339] J. Li, On neighborhood condition for graphs to have $[a, b]$-factors, Discrete Math. 260 (2003) (1-3) 217-221.

[340] Y. Li, On small treewidth problem and algorithm of matching extension, $\mathrm{Ph}$. D. Dissertation, Zhongshan University, 2007.

[341] G. Li, C. Chen and G. Yu, Orthogonal factorizations of graphs, Discrete Math. 245 (2002) (1-3) 173-194.

[342] G. Li and Z. Liu, On connected factors in $k_{1,3}$-free graphs, Acta Mathematicae Applicatae Sinica (English Series) 14 (1998) (1) 43-47.

[343] G. Li and Z. Liu, ( $g, f)$-factorizations orthogonal to a subgraph in graphs, Sci. China Ser. A 41 (1998) (3) 267-272. 
[344] G. Li, Y. Xu, C. Chen and Z. Liu, On connected $[g, f+1]$-factors in graphs, Combinatorica 25 (2005) (4) 393-405.

[345] G. Li, B. Zhu and C. Chen, On connected $[k, k+1]$-factors in claw-free graphs, Ars Combin. 62 (2002) 207-219.

[346] H. Li and G. Wang, Color degree and heterochromatic matchings in edge-colored bipartite graphs, (manuscript).

[347] J. Li and Y. Ma, Complete-factors and $(g, f)$-factors, Discrete Math. 265 (2003) (1-3) 385-391.

[348] Y. Li and M. Cai, A degree condition for the existence of connected factors, Australas. J. Combin. 14 (1996) 77-83.

[349] Y. Li and M. Cai, A degree condition for a graph to have $[a, b]$-factors, J. Graph Theory 27 (1998) (1) 1-6.

[350] C. Liang and Y. Liu, ID-factor-critical Claw-free Graphs, (manuscript), 2006.

[351] N. B. Limaye and D. G. Sarvate, On $r$-extendability of the hypercube $Q_{n}$, Math. Bohem. 122 (1997) (3) 249-255.

[352] H. Lin and X. Guo, On $(2 n+1)$-contractible graphs and 2n-pairs contractible graphs, (manuscript).

[353] C. H. C. Little, A theorem on connected graphs in which every edge belongs to a 1-factor, J. Aust. Math. Soc. 18 (1974) 450-452.

[354] C. H. C. Little, D. D. Grant and D. A. Holton, On defect-d matchings in graphs, Discrete Math. 13 (1975) (1) 41-54.

[355] C. H. C. Little and F. Rendl, An algorithm for the ear decomposition of a 1-factor covered graph, J. Aust. Math. Soc. 46 (1989) (2) 296-301.

[356] C. H. C. Little, F. Rendl and I. Fischer, Towards a characterisation of Pfaffian near bipartite graphs, Discrete Math. 244 (2002) (1-3) 279-297.

[357] G. Liu, On ( $g, f)$-covered graphs, Acta Math. Sci. Ser. B Engl. Ed. 8 (1988) (2) 181-184.

[358] G. Liu, On [a,b]-covered graphs, J. Combin. Math. Combin. Comput. 5 (1989) 14-22.

[359] G. Liu, On $f$-covered Graphs, Seventeenth Manitoba Conference on Numerical Math. and Computing (Winnipeg, MB, 1987), Congress. Numer. 61 (1988) 8186. 
[360] G. Liu, Orthogonal ( $g, f)$-factorizations in graphs, Discrete Math. 143 (1995) (13) $153-158$.

[361] G. Liu and P. Li, Minimum fractional $(g, f)$-factors of graphs, Graphs Combin., (to appear).

[362] G. Liu and J. Wang, ( $a, b, k)$-critical graphs, Chinese Sci. Bull. 42 (1997) (17) 1492-1493.

[363] G. Liu and J. Wang, (a,b,k)-critical graphs, Adv. Math. (China) 27 (1998) (6) 536-540.

[364] G. Liu and Q. Yu, Generalization of matching extensions in graphs, Discrete Math. 231 (2001) (1-3) 311-320.

[365] G. Liu and Q. Yu, On n-edge-deletable and n-critical graphs, Bull. Inst. Combin. Appl. 24 (1998) 65-72.

[366] G. Liu and Q. Yu, On $(n, k)$-extendable graphs and induced subgraphs, International Math. Forum 23 (2007) 1141-1148.

[367] G. Liu and Q. Yu, Strongly pan-factorial property in cages, Ars Combin. (to appear).

[368] G. Liu and Q. Yu, Toughness and perfect matching in graphs, Ars Combin. 48 (1998) 129-134.

[369] G. Liu and Q. Yu, $k$-factors and extendability with prescribed components, Congr. Numer. 139 (1999) 77-88.

[370] G. Liu, Q. Yu and L. Zhang, Maximum fractional factors in graphs, Appl. Math. Lett. 20 (2007) (12) 1237-1243.

[371] G. Liu and W. Zang, f-factors in bipartite $(m f)$-graphs, Discrete Appl. Math. 136 (2004) (1) 45-54.

[372] G. Liu and L. Zhang, Characterizations of maximum fractional $(g, f)$-factors of graphs, Disc. Appl. Math. (to appear).

[373] G. Liu and L. Zhang, Factors and factorization of graphs, Adv. Math. (China) 29 (2000) (4) 289-296.

[374] G. Liu and L. Zhang, Fractional $(g, f)$-factors of graphs, Acta Math. Sci. Ser. B Engl. Ed. 21 (2001) (4) 541-545.

[375] G. Liu and L. Zhang, Maximum fractional (0,f)-factors of graphs, Math. Appl. (Wuhan) 13 (2000) (1) 31-35. 
[376] G. Liu and L. Zhang, Properties of fractional k-factors of graphs, Acta Math. Sci. Ser. B Engl. Ed. 25 (2005) (2) 301-304.

[377] G. Liu and L. Zhang, Toughness and the existence of fractional $k$-factors of graphs, Discrete Math. 308 (2008) 1741-1748.

[378] G. Liu and X. Zhang, Fractional factors and fractional Hamilton graphs, Adv. Math. (China) 35 (2006) (3) 257-264.

[379] G. Liu and B. Zhu, Some problems on factorizations with constraints in bipartite graphs, Discrete Appl. Math. 128 (2003) (2-3) 421-434.

[380] J. Liu and Q. Yu, Matching extensions and products of graphs, in J. W. K. John Gimbel and L. V. Quintas (Eds.) Quo Vadis, Graph Theory? - A Source Book for Challenges and Directions, vol. 55, Elsevier, 1993, 191-200.

[381] J. Liu and H. Zhou, Maximum induced matchings in graphs, Discrete Math. 170 (1997) (1-3) 277-281.

[382] Y. Liu and J. Hao, The enumeration of near-perfect matchings of factor-critical graphs, Discrete Math. 243 (2002) (1-3) 259-266.

[383] Y. Liu and G. Liu, The fractional matching numbers of graphs, Networks 40 (2002) (4) 228-231.

[384] Y. Liu and G. Liu, Number of maximum matchings of bipartite graphs with positive surplus, Discrete Math. 274 (2004) (1-3) 311-318.

[385] Y. Liu and Y. Ma, Maximal Non-ID-factor-critical Graphs, J. of Math. Study 36 (2003) 374-378.

[386] Z. Lonc and Z. Ryjáček, Factors of claw-free graphs, Czechoslovak Math. J. 41 (1991) 120-130.

[387] D. Lou, Some conditions for $n$-extendable graphs, Australas. J. Combin. 9 (1994) 123-136.

[388] D. Lou, A local independence number condition for $n$-extendable graphs, Discrete Math. 195 (1999) (1-3) 263-268.

[389] D. Lou, Characterizing minimally $n$-extendable bipartite graphs, Discrete Math. 308 (2008) (11) 2269-2272.

[390] D. Lou, On 2-extendable planar graphs, J. of Zhongshan University, (in Chinese), 29 (1990) 124-126.

[391] D. Lou, On local neighborhood condition of $n$-extendable graphs, (in Chinese) Ke Xui Tong Bao, 41 (1996) 1899-1901. 
[392] D. Lou, On the structure of minimally $n$-extendable bipartite graphs, Discrete Math. 202 (1999) (1-3) 173-181.

[393] D. Lou, On matchability of graphs, Australas. J. Combin. 21 (2000) 201-210.

[394] D. Lou and D. A. Holton, Lower bound of cyclic edge connectivity for nextendability of regular graphs, Discrete Math. 112 (1993) (1-3) 139-150.

[395] D. Lou and D. Rao, Characterizing factor critical graphs and an algorithm, Australas. J. Combin. 30 (2004) 51-56.

[396] D. Lou and D. Rao, Graphs with no $M$-alternating path between two vertices, Discrete Math. 280 (2004) (1-3) 237-240.

[397] D. Lou, A. Saito and L. Teng, A note on internally disjoint alternating paths in bipartite graphs, Discrete Math. 290 (2005) (1) 105-108.

[398] D. Lou, A. Saito and L. Teng, To determine 1-extendable graphs and its algorithm, Ars Combin. 69 (2003) 223-228.

[399] D. Lou and Q. Yu, Connectivity of $k$-extendable graphs with large $k$, Discrete Appl. Math. 136 (2004) (1) 55-61.

[400] D. Lou and Q. Yu, Sufficient conditions for n-matchable graphs, Australas. J. Combin. 29 (2004) 127-133.

[401] D. Lou and N. Zhong, A highly efficient algorithm to determine bicritical graphs, Proc. of the 7th Annual International Conference on Computing and Combin., Lecture Notes in Computer Science 2108 (2001) 349-356.

[402] D. Lou and Q. Zhu, The 2-extendability of strongly regular graphs, Discrete Math. 148 (1996) (1-3) 133-140.

[403] L. Lovász, Generalized factors of graphs, Combinatorial theory and its applications, II, (Proc. Colloq., Balatonfured, 1969), North-Holland, Amsterdam (1970), 773-781.

[404] L. Lovász, Subgraphs with prescribed valencies, Journal of Combinatorial Theory 8 (1970) (4) 391-416.

[405] L. Lovász, The factorization of graphs. II, Acta Math. Hungar. 23 (1972) (1) $223-246$.

[406] L. Lovász, On the structure of factorizable graphs, Acta Math. Hungar. 23 (1972) (1) 179-195.

[407] L. Lovász, On the structure of factorizable graphs. II, Acta Math. Hungar. 23 (1972) (3) 465-478. 
[408] L. Lovász, Antifactors of graphs, Period. Math. Hungar. 4 (1973) (2) 121-123.

[409] L. Lovász, A note on factor-critical graphs, Studia Sci. Math. Hungar. 7 (1973) 279-280.

[410] L. Lovász, Valencies of graphs with 1-factors, Period. Math. Hungar. 5 (1974) (2) 149-151.

[411] L. Lovász, Combinatorial problems and exercises, Amsterdam-New YorkOxford: North-Holland Publishing Company. Budapest: Akademiai Kiado, Publishing House of the Hungarian Academy of Sciences, 1979.

[412] L. Lovász, Ear-decompositions of matching-covered graphs, Combinatorica 3 (1983) (1) 105-117.

[413] L. Lovász, Matching structure and the matching lattice, J. Combin. Theory Ser. B 43 (1987) (2) 187-222.

[414] L. Lovász and M. D. Plummer, Matching theory, Annals of Discrete Mathematics, Vol. 29. North-Holland Mathematics Studies, Vol. 121, Amsterdam etc.: North-Holland. 1986.

[415] L. Lovász and M. D. Plummer, On a family of planar bicritical graphs, Proc. London Math. Soc. s3-30 (1975) (2) 160-176.

[416] L. Lovász and M. D. Plummer, On minimal elementary bipartite graphs, J. Combin. Theory Ser. B 23 (1977) (1) 127-138.

[417] L. Lovász and M. D. Plummer, On bicritical graphs, in Infinite and finite sets, North-Holland, 1975, 1051-1079.

[418] L. Lovász and M. D. Plummer, Some recent results on graph matching, Graph Theory and Its Applications East and West: Proceedings of the First ChinaUSA International Graph Theory Conference, Annals of the New York Academy of Sciences, 576 (1989) 389-398.

[419] V. V. Lozin, On maximum induced matchings in bipartite graphs, Inform. Process. Lett. 81 (2002) (1) 7-11.

[420] C. L. Lucchesi and M. H. de Carvalho, Base for the matching lattice of matching covered graphs, Technical Report DCC 95-09.

[421] Y. Ma and G. Liu, Fractional factors and isolated toughness of graphs, Math. Appl. (Wuhan) 19 (2006) 188-194.

[422] Y. Ma and G. Liu, Isolated toughness and the existence of fractional factors, Acta Math. Appl. Sin. 26 (2003) (1) 133-140. 
[423] W. Mader, Über die anzahl der von den 1-faktoren eines graphen überdeckten ecken, Math. Nachr. 56 (1973) 195-200.

[424] E. Mahmoodian, On factors of a graph, Canad. J. Math. 29 (1977) 438-440.

[425] P. Maschlanka and L. Volkmann, Independence number in $n$-extendable graphs, Discrete Math. 154 (1996) (1-3) 167-178.

[426] H. Matsuda, A neighborhood condition for graphs to have [a,b]-factors, Discrete Math. 224 (2000) (1-3) 289-292.

[427] H. Matsuda, A neighborhood condition for graphs to have $[a, b]$-factors II, Graphs Combin. 18 (2002) (4) 763-768.

[428] H. Matsuda, Degree conditions for the existence of $[k, k+1]$-factors containing a given Hamiltonian cycle, Australas. J. Combin. 26 (2002) 273-281.

[429] H. Matsuda, Degree conditions for Hamiltonian graphs to have $[a, b]$-factors containing a given Hamiltonian cycle, Discrete Math. 280 (2004) (1-3) 241-250.

[430] H. Matsuda, Fan-type results for the existence of $[a, b]$-factors, Discrete Math. 306 (2006) (7) 688-693.

[431] H. Matsuda, On 2-edge-connected $[a, b]$-factors of graphs with Ore-type condition, Discrete Math. 296 (2005) (2-3) 225-234.

[432] H. Matsuda, Ore-type conditions for the existence of even [2, b]-factors in graphs, Discrete Math. 304 (2005) (1-3) 51-61.

[433] W. McCuaig, Brace generation, J. Graph Theory 38 (2001) (3) 124-169.

[434] W. McCuaig, Even dicycles, J. Graph Theory 35 (2000) (1) 46-68.

[435] C. McDiarmid and B. Reed, Almost every graph can be covered by $\left\lceil\frac{\Delta}{2}\right\rceil$ linear forests, Combin. Probab. Comput. 4 (1995) (3) 257-268.

[436] K. McDougal, On asymmetric $(0,1)$-matrices with given row and column sum vectors, Discrete Math. 137 (1995) (1-3) 377-381.

[437] A. McGregor-Macdonald, The $E(m, n)$ property, M.S. Thesis, Univ. of Otago, Dunedin, New Zealand, 2000.

[438] N. S. Mendelsohn and A. L. Dulmage, Some generalizations of the problem of distinct representatives, Canad. J. Math. 10 (1958) 230-241.

[439] A. A. A. Miranda and C. L. Lucchesi, A polynomial time algorithm for recognizing near-bipartite pfaffian graphs, Electron. Notes Discrete Math. 30 (2008)171176. 
[440] V. Mkrtchyan, A note on minimal matching covered graphs, Discrete Math. 306 (2006) (4) 452-455.

[441] U. S. R. Murty, An application of Rado's theorem to disjoint matchings in bipartite graphs, J. London Math. Soc. (2) 17 (1978) (2) 193-194.

[442] D. Naddef and W. R. Pulleyblank, Matchings in regular graphs, Discrete Math. 34 (1981) (3) 283-291.

[443] D. J. Naddef and W. R. Pulleyblank, Ear decompositions of elementary graphs and GF(2)-rank of perfect matchings, in M. G. Achim Bachem and B. Korte (Eds.) Bonn Workshop on Combinatorial Optimization, vol. 66, North-Holland, 1982, 241-260.

[444] Y. Nam, Ore-type condition for the existence of connected factors, J. Graph Theory 56 (2007) (3) 241-248.

[445] L. Nebeský, Some sufficient conditions for the existence of a 1-factor, J. Graph Theory 2 (1978) (3) 251-255.

[446] V. Neumann-Lara and E. Rivera-Campo, Spanning trees with bounded degrees, Combinatorica 11 (1991) (1) 55-61.

[447] L. Ng and M. Schultz, k-ordered Hamiltonian graphs, J. Graph Theory 24 (1997) (1) 45-57.

[448] T. Niessen, A characterization of graphs having all $(g, f)$-factors, J. Combin. Theory Ser. B 72 (1998) (1) 152-156.

[449] T. Niessen, Minimum degree, independence number and regular factors, Graphs Combin. 11 (1995) (4) 367-378.

[450] T. Niessen, Nash-Williams conditions and the existence of $k$-factors, Ars Combin. 34 (1992) 251-256.

[451] T. Niessen and B. Randerath, Regular factors of simple regular graphs and factor-spectra, Discrete Math. 185 (1998) (1-3) 89-103.

[452] Q. Ning, On T-joins and odd factors, Oper. Res. Lett. 6 (1987) (3) 135-138.

[453] T. Nishimura, A closure concept in factor-critical graphs, Discrete Math. 259 (2002) (1-3) 319-324.

[454] T. Nishimura, A degree condition for the existence of $k$-factors, J. Graph Theory 16 (1992) (2) 141-151.

[455] T. Nishimura, A new recursive theorem on n-extendibility, Graphs Combin. 13 (1997) (1) 79-83. 
[456] T. Nishimura, A theorem on n-extendable graphs, Ars Combin. 38 (1994) 3-5.

[457] T. Nishimura, Component factors and induced subgraphs, J. Graph Theory 22 (1996) (4) 305-308.

[458] T. Nishimura, Degree factors of line graphs, Ars Combin. 38 (1994) 149-159.

[459] T. Nishimura, Independence number, connectivity, and degree factors, SUT J. Math. 25 (1989) (1) 79-87.

[460] T. Nishimura, Independence number, connectivity, and $r$-factors, J. Graph Theory 13 (1989) (1) 63-69.

[461] T. Nishimura, Note on a closure concept and matching extension, Australas. J. Combin. 32 (2005) 171-175.

[462] T. Nishimura, On 1-factors and matching extension, Discrete Math. 222 (2000) (1-3) 285-290.

[463] T. Nishimura, Regular factors of line graphs, Discrete Math. 85 (1990) (2) 215219.

[464] T. Nishimura, Regular factors of line graphs. II, Math. Japon. 36 (1991) (6) 1033-1040.

[465] T. Nishimura and A. Saito, Two recursive theorems on $n$-extendibility, Discrete Math. 162 (1996) (1-3) 319-323.

[466] T. Nishizeki, On the relationship between the genus and the cardinality of the maximum matchings of a graph, Discrete Math. 25 (1979) (2) 149-156.

[467] T. Nishizeki and I. Baybars, Lower bounds on the cardinality of the maximum matchings of planar graphs, Discrete Math. 28 (1979) (3) 255-267.

[468] S. Norine, C. H. C. Little and K. L. Teo, A new proof of a characterisation of Pfaffian bipartite graphs, J. Combin. Theory Ser. B 91 (2004) (1) 123-126.

[469] S. Norine and R. Thomas, Generating bricks, J. Combin. Theory Ser. B 97 (2007) (5) 769-817.

[470] S. Norine and R. Thomas, Minimal bricks, J. Combin. Theory Ser. B 96 (2006) (4) 505-513.

[471] O. Ore, Graphs and subgraphs, Trans. Amer. Math. Soc. 84 (1957) 109-136.

[472] O. Ore, Note on Hamilton circuits, Amer. Math. Monthly 67 (1960) (1) 55. 
[473] K. Ota and T. Tokuda, A degree condition for the existence of regular factors in $K_{1, n}$-free graphs, J. Graph Theory 22 (1996) (1) 59-64.

[474] V. Patel, Union of perfect matchings in cubic graphs and implications of the Berge-Fulkerson conjecture, CDAM Research Report LSE-CDAM-2006-06, 2006.

[475] J. Peterson, Die Theorie der regulären Graphen, Acta Math. 15 (1891) 193-220.

[476] M. Plantholt and S. K. Tipnis, All regular multigraphs of even order and high degree are 1-factorable, Electron. J. Combin. 8 (2001) (1) R41.

[477] M. J. Plantholt and S. K. Tipnis, Regular multigraphs of high degree are 1factorizable, J. London Math. Soc. (2) 44 (1991) (3) 393-400.

[478] J. Plesník, Connectivity of regular graphs and the existene of 1-factors, Mat. Ćasop. 22 (1972) 310-318.

[479] J. Plesník, Remarks on rgular factors of regular graphs, Czechoslovak Math. J. 24 (1974) 292-300.

[480] M. D. Plummer, 2-extendability in two classes of claw-free graphs, Graph Theory, Combinatorics and Applications, Vol. 2, Eds.: Y. Alavi and A. Schwenk, John Wiley \& Sons, New York, 1995, 905-922.

[481] M. D. Plummer, A theorem on matchings in the plane, in C. T. B. T. Lars Dovling Andersen, Ivan Tafteberg Jakobsen and P. D. Vestergaard (Eds.) Graph Theory in Memory of G.A. Dirac, vol. 41, Elsevier, 1988, 347-354.

[482] M. D. Plummer, Degree sums, neighborhood unions and matching extension in graphs, in: R. Bodendiek, ed., Contemporary Methods in Graph Theory (B. I. Wissensch-aftsverlag, Mannheim, 1990) 489-502.

[483] M. D. Plummer, Extending matchings in claw-free graphs, Discrete Math. 125 (1994) (1-3) 301-307.

[484] M. D. Plummer, Extending matchings in graphs: A survey, Discrete Math. 127 (1994) (1-3) 277-292.

[485] M. D. Plummer, Extending matchings in graphs: an update, Congr. Numer. 116 (1996) 3-32.

[486] M. D. Plummer, Extending matchings in planar graphs IV, Discrete Math. 109 (1992) (1-3) 207-219.

[487] M. D. Plummer, Extending matchings in planar graphs V, Discrete Math. 150 (1996) (1-3) 315-324. 
[488] M. D. Plummer, Graph factors and factorization: 1985-2003: A survey, Discrete Math. 307 (2007) (7-8) 791-821.

[489] M. D. Plummer, Matching extension in bipartite graphs, Congr. Numer. 54 (1986) 245-258.

[490] M. D. Plummer, Matching extension and connectivity in graphs, Congress. Numer. 63 (1988) 147-160.

[491] M. D. Plummer, Matching extension and the genus of a graph, J. Combin. Theory Ser. B 44 (1988) (3) 329-337.

[492] M. D. Plummer, Matching extension in regular graphs, Graph Theory, Combinatorics, Algorithms and Applications, SIAM, Philadelphia, 1991, 416-424.

[493] M. D. Plummer, Matching theory-a sampler: from dénes könig to the present, Discrete Math. 100 (1992) (1-3) 177-219.

[494] M. D. Plummer, On n-extendable graphs, Discrete Math. 31 (1980) (2) 201-210.

[495] M. D. Plummer, Toughness and matching extension in graphs, Discrete Math. 72 (1988) (1-3) 311-320.

[496] M. D. Plummer and A. Saito, Closure and factor-critical graphs, Discrete Math. 215 (2000) (1-3) 171-179.

[497] M. D. Plummer and A. Saito, Forbidden subgraphs and bounds on the size of a maximum matching, J. Graph Theory 50 (2005) (1) 1-12.

[498] M. D. Plummer and X. Zha, On the $p$-factor-criticality of the Klein bottle, Discrete Math. 287 (2004) (1-3) 171-175.

[499] M. I. Porteous, Generalizing matching extension, M. A. Thesis, Univ. of Otago, 1995

[500] M. I. Porteous and R. E. L. Aldred, Matching extensions with prescribed and forbidden edges, Australas. J. Combin. 13 (1996) 163-174.

[501] C. R. Pranesachar, A class of 'matching-equivalent' bipartite graphs, Discrete Math. 203 (1999) (1-3) 207-213.

[502] W. R. Pulleyblank, Faces of matching polyhedra, University of Waterloo, Ph. D. Thesis, 1973.

[503] W. R. Pulleyblank, Fractional matchings and the Edmonds-Gallai theorem, Discrete Appl. Math. 16 (1987) (1) 51-58. 
[504] W. R. Pulleyblank, Matching and extensions, Handbook of Combinatorics, Elsevier, 1995, 179-232.

[505] W. R. Pulleyblank, Minimum node covers and 2-bicritical graphs, Math. Program. 17 (1979) (1) 91-103.

[506] J. Qian, Induced matching extendable graph powers, Graphs Combin. 22 (2006) (3) 391-398.

[507] D. Rautenbach and L. Volkmann, Independent domination and matchings in graphs, Discrete Math. 259 (2002) (1-3) 325-330.

[508] A. M. Robertshaw and D. R. Woodall, Binding number conditions for matching extension, Discrete Math. 248 (2002) (1-3) 169-179.

[509] Z. Ryjáček, Almost claw-free graphs, J. Graph Theory 18 (1994) (5) 469-477.

[510] Z. Ryjáček, Factors and circuits in $K_{l, 3}$-free graphs, in: Combinatorics and Graph Theory, Vol. 25, Polish Scientific Publishers, Warsaw, Banach Centre Publications, 1989, 137-145.

[511] Z. Ryjáček, Matching extension in $K_{1, r}$-free graphs with independent claw centers, Discrete Math. 164 (1997) (1-3) 257-263.

[512] Z. Ryjáček, A. Saito and R. H. Schelp, Closure, 2-factors, and cycle coverings in claw-free graphs, J. Graph Theory 32 (1999) (2) 109-117.

[513] H. A. B. Saip and C. L. Lucchesiy, Matching algorithms for bipartite graphs, manuscript.

[514] A. Saito, One-factors and k-factors, Discrete Math. 91 (1990) (3) 323-326.

[515] A. Saito and M. Watanabe, Partitioning graphs into induced stars, Ars Combin. 36 (1993) 3-6.

[516] D. P. Sanders, On paths in planar graphs, J. Graph Theory 24 (1997) (4) 341345.

[517] G. N. Sárközy, On 2-factors with $k$ components, (manuscript).

[518] E. R. Scheinerman and D. H. Ullman, Fractional graph theory, John Wiley and Sons, Inc., New York, 1997.

[519] T. Schönberger, Ein Beweis des Petersenschen Graphensatzes, Acta Litt. Acad. Sci. Szeged 7 (1934) 51-57.

[520] J. Schönheim and A. Bialostocki, Decomposition of $K_{n}$ into subgraphs of prescribed type, Arch. Math. (Basel) 31 (1978) (1) 105-112. 
[521] G. Schrag and L. Cammack, On the 2-extendability of the generalized Petersen graphs, Discrete Math. 78 (1989) (1-2) 169-177.

[522] A. Schrijver, Bounds of permanents, and the number of 1-factor and 1factorizations of bipartite graphs, Surveys in Combinatorics, London Math. Soc. Lecture Note Ser. 82 (1983), 107-134.

[523] A. Schrijver, Counting 1-factors in regular bipartite graphs, J. Combin. Theory Ser. B 72 (1998) (1) 122-135.

[524] A. Schrijver, Matching, Edge-Colouring, Dimers, manucript.

[525] A. Sebö, General antifactors of graphs, J. Combin. Theory Ser. B 58 (1993) (2) $174-184$.

[526] J. C. Setubal, New experimental results for bipartite matching, (manuscript).

[527] P. D. Seymour, On multicolourings of cubic graphs, and conjectures of Fulkerson and Tutte, Proc. London Math. Soc. 38 (1979) 423-460.

[528] K. W. Shavo, A result on extendibility in the powers of graphs, J. of Graph Theory, 56 (2007) 1-22.

[529] M. Shi, X. Yuan, M.-c. Cai and O. Favaron, $(3, k)$-factor-critical graphs and toughness, Graphs Combin. 15 (1999) (4) 463-471.

[530] H. Shirazi and J. Verstraëte, A note on polynomials and f-factors of graphs, manuscript, 2007.

[531] B. Spille and L. Szegö, A Gallai-Edmonds-type structure theorem for pathmatchings, J. Graph Theory 46 (2004) (2) 93-102.

[532] K. Steffens, Maximal tight sets and the Edmonds-Gallai decomposition for matchings, Combinatorica 5 (1985) (4) 359-365.

[533] A. Steger and M.-l. Yu, On induced matchings, Discrete Math. 120 (1993) (1-3) $291-295$.

[534] M. Stehlík, A generalisation of matching and colouring, Discrete Math. 285 (2004) (1-3) 257-265.

[535] R. A. Stong, On 1-factorizability of Cayley graphs, J. Combin. Theory Ser. B 39 (1985) (3) 298-307.

[536] R. A. Stong, Orthogonal matchings, Discrete Math. 256 (2002) (1-2) 513-518.

[537] H. Su and H. Zhang, The factor-criticality of surfaces, (manusctript). 
[538] D. P. Sumner, 1-factors and antifactor sets, J. London Math. Soc. (2) 13 (1976) (2) 351-359.

[539] D. P. Sumner, Graphs with 1-factors, Proc. Amer. Math. Soc. 42 (1974) (1) $8-12$.

[540] D. P. Sumner, On Tutte's factorization theorem, Graphs and Combinatorics (Proc. Capital Conf., George Washington Univ., Washington, D.C., 1973, Vol. 406, Springer, Berlin, (1974) 350-355.

[541] D. P. Summer, Randomly matchable graphs, J. Graph Theory 3 (1979) (2) $183-186$.

[542] D. P. Sumner, Subtrees of a graph and the chromatic number, The Theory and Applications of Graphs, Wiley, New York, 1981, 557-576.

[543] Z. Szigeti, On a matroid defined by ear-decompositions of graphs, Combinatorica 16 (1996) (2) 233-241.

[544] Z. Szigeti, On generalizations of matching-covered graphs, European J. Combin. 22 (2001) (6) 865-877.

[545] Z. Szigeti, The two ear theorem on matching-covered graphs, J. Combin. Theory Ser. B 74 (1998) (1) 104-109.

[546] Z. Szigeti, Perfect matchings versus odd cuts, Combinatorica 22 (2002) (4) $575-589$.

[547] R. Thomas, A survey of Pfaffian orientations of graphs, Proceedings of the International Congress of Mathematicians, Madrid, Spain, 2006.

[548] C. Thomassen, A remark on the factor theorems of Lovász and Tutte, J. Graph Theory 5 (1981) (4) 441-442.

[549] C. Thomassen, Graph decomposition with constraints on the connectivity and minimum degree, J. Graph Theory 7 (1983) (2) 165-167.

[550] C. Thomassen, Tilings of the Torus and the Klein bottle and vertex-transitive graphs on a fixed surface, Trans. Amer. Math. Soc. 323 (1991) (2) 605-635.

[551] F. Tian, A short proof of Fan's theorem, Discrete Math. 286 (2004) (3) 285-286.

[552] F. Tian, B. Wei and L. Zhang, Hamiltonicity in 3-domination-critical graphs with $\alpha=\delta+2$, Discrete Appl. Math. 92 (1999) (1) 57-70.

[553] T. Tokuda, A degree condition for the existence of $[a, b]$-factors in $K_{1, n}$-free graphs, Tokyo J. Math. 7 (1998) 377-380. 
[554] T. Tokuda, Connected $[a, b]$-factors in $K_{1, n}$-free graphs containing an $[a, b]$ factor, Discrete Math. 207 (1999) (1-3) 293-298.

[555] T. Tokuda, Connected factors in $K_{1, n}$-free graphs containing an $[a, b]$-factor, Discrete Math. 306 (2006) (21) 2806-2810.

[556] T. Tokuda, B. Xu and J. Wang, Connected factors and spanning trees in graphs, Graphs Combin. 19 (2003) (2) 259-262.

[557] N. Tokushige, Binding number and minimum degree for $k$-factors, J. Graph Theory 13 (1989) (5) 607-617.

[558] E. Tomová, On the decomposition of the complete directed graph into factors with given diameters, Mat. Časopis Sloven. Akad. Vied 20 (1970) 257-261.

[559] J. Topp and P. D. Vestergaard, Odd factors of a graph, Graphs Combin. 9 (1993) (4) 371-381.

[560] J. Topp and P. D. Vestergaard, Totally equimatchable graphs, Discrete Math. 164 (1997) (1-3) 285-290.

[561] M. Truszczyński, Linear arboricity of graphs, Math. Slovaca 36 (1986) 105-108.

[562] N. Tsuyoshi, Independence number, connectivity, and r-factors, J. Graph Theory 13 (1989) 63-69.

[563] W. T. Tutte, The factorization of linear graphs, J. London Math. Soc. (2) 22 (1947) (2) 107-111.

[564] W. T. Tutte, The factors of graphs, Canad. J. Math. 4 (1952) 314-328.

[565] W. T. Tutte, The 1-factors of oriented graphs, Proc. Amer. Math. Soc. 4 (1953) 922-931.

[566] W. T. Tutte, A short proof of the factor theorem for finite graphs, Canad. J. Math. 6 (1954) 347-352.

[567] W. T. Tutte, Spanning subgraphs with specified valencies, Discrete Math. 9 (1974) (1) 97-108.

[568] W. T. Tutte, The subgraph problem, in B. Bollobás (Ed.) Advances in Graph Theory, vol. 3, Elsevier, 1978, 289-295.

[569] W. T. Tutte, Graph factors, Combinatorica 1 (1981) (1) 79-97.

[570] V. V. Vazirani and M. Yannakakis, Pfaffian orientations, 0-1 permanents, and even cycles in directed graphs, Discrete Appl. Math. 25 (1989) 179-190. 
[571] L. Volkmann, A short proof of a theorem of Kano and Yu on factors in regular graphs, Electron. J. Combin. 14 (2007) (1) N10.

[572] L. Volkmann, On regular $(1, q)$-extendable graphs, manuscript.

[573] L. Volkmann, Regular graphs, regular factors, and the impact of Petersen's theorems, Jahresber. Deutsch. Math.-Verein. 97 (1995) (1) 19-42.

[574] L. Volkmann, The maximum size of graphs with a unique $k$-factor, Combinatorica 24 (2004) (3) 531-540.

[575] L. Volkmann, The Petersen graph is not 1-factorable: postscript to the Petersen graph is not 3-edge-colorable-a new proof' [discrete math. 268 (2003) 325-326], Discrete Math. 287 (2004) (1-3) 193-194.

[576] M. Šajna, On the problem of a matching orthogonal to a 2-factorization, Discrete Math. 218 (2000) (1-3) 277-282.

[577] M. Štehlík, A hypergraph version of the Gallai-Edmonds theorem, Electron. Notes Discrete Math. 28 (2007) 387-391.

[578] K. L. Walcher, Matching extension in the powers of $n$-connected graphs, $J$. Graph Theory 23 (1996) (4) 355-360.

[579] C. Wang, A degree condition for the existence of $k$-factors with prescribed properties, Int. J. Math. Comput. Sci. 6 (2005) 863-873,

[580] Q. Wang and J. Yuan, 4-regular claw-free IM-extendable graphs, Discrete Math. 294 (2005) (3) 303-309.

[581] Q. Wang and J. Yuan, Maximal IM-unextendable graphs, Discrete Math. 240 (2001) (1-3) 295-298.

[582] T. Wang and Q. Yu, A conjecture on $k$-factor-critical and 3 - $\gamma$-critical graphs, (submit).

[583] T. Wang and Q. Yu, Factor-critical property in 3-dominating-critical graphs, Discrete Math. (in Press).

[584] X. Wang and Y. Lin, Extremal Graphs about Bipartite Matching Extendability, (manuscript).

[585] X. Wang, Z. Zhang and Y. Lin, Bipartite matching extendable graphs, (manuscript).

[586] B. Wei and Y. Zhu, Hamiltonian $k$-factors in graphs, J. Graph Theory 25 (1997) (3) 217-227. 
[587] X. Wen, Researches on defect $n$-extendable graphs and the OHP problem of Halin graphs, Ph. D. Dissertation, Zhongshan University, 2008.

[588] X. Wen and D. Lou, Characterizing defect $n$-extendable graphs and $(2 n+1)$ critical graphs, (manuscript).

[589] X. Wen and D. Lou, Path decomposition of defect 1-extendable bipartite graphs, Australas. J. Combin. 39 (2007) 175-182.

[590] H. Whitney, Non-separable and planar graphs, Trans. Amer. Math. Soc. 34 (1932) (2) 339-362.

[591] S. Win, A sufficient condition for a graph to contain three disjoint 1-factors, $J$. Graph Theory 6 (1982) (4) 489-492.

[592] S. Win, On a connection between the existence of $k$-trees and the toughness of a graph, Graphs Combin. 5 (1989) (1) 201-205.

[593] D. R. Woodall, The binding number of a graph and its Anderson number, $J$. Combin. Theory Ser. B 15 (1973) (3) 225-255.

[594] D. R. Woodall, $k$-factors and neighbourhoods of independent sets in graphs, $J$. London Math. Soc. (2) 41 (1990) (3) 385-392.

[595] J.-L. Wu, On the linear arboricity of planar graphs, J. Graph Theory 31 (1999) (2) 129-134.

[596] Z. Wu, X. Yang and Q. Yu, Factor-criticality of Cartesian Product, (preprint).

[597] B. Xu, Z. Liu and T. Tokuda, Connected factors in $K_{1, n}$-free graphs containing a $(g, f)$-factor, Graphs Combin. 14 (1998) (4) 393-395.

[598] R. Xu, Factor, Connected Factor and Extendability of Graphs. Ph.D. Thesis, Shandong University, 1999.

[599] R. Xu, Some Ore-type conditions for the existence of connected $[2, k]$-factors in graphs, Discrete Math. 194 (1999) (1-3) 295-298.

[600] R. Xu and Q. Yu, (4,k)-factor-critical graphs and toughness, Advances in Math. 28 (1999) 539-540.

[601] R. Xu and Q. Yu, Degree-sum conditions for $k$-extendable graphs, Congr. Numer. 163 (2003) 189-195.

[602] R. Xu and Q. Yu, Note on toughness and (k,r)-factor-critical graphs, Bull. Inst. Combin. Appl. 29 (2000) 85-87. 
[603] R. Xu and Q. Yu, Ore-type conditions for the existence of $k$-factors with prescribed properties, Congr. Numer. 162 (2003) 97-109.

[604] R. Xu and Q. Yu, Some conditions for $k$-maximal/minimal graphs, (unpublished manuscript) 2002.

[605] J. Yan and G. Liu, Vertex-disjoint quadrilaterals in bipartite graphs, J. Systems Science and Complexing 17 (2004) (4) 532-537.

[606] G. Yan, A new result on Alspach's problem, Graphs Combin. 15 (1999) (3) 365-371.

[607] G. Yan, Some new results on $(g, f)$-factorizations of graphs, J. Combin. Math. Combin. Comput. 18 (1995) 177-185.

[608] G. Y. Yan, J. F. Pan, C. K. Wong and T. Tokuda, Decomposition of graphs into ( $g, f)$-factors, Graphs Combin. 16 (2000) (1) 117-126.

[609] W. Yan, Y. N. Yeh and F. Zhang, Enumeration of perfect matchings of graphs with rotational symmetry by Pfaffians, (manuscript).

[610] W. Yan and F. Zhang, Enumeration of perfect matchings of graphs with reflective symmetry by Pfaffians, Adv. in Appl. Math. 32 (2004) (4) 655-668.

[611] W. Yan and F. Zhang, Enumeration of perfect matchings of a type of cartesian products of graphs, Discrete Appl. Math. 154 (2006) (1) 145-157.

[612] J. Yang, Y. Ma and G. Liu, Fractional $(g, f)$-factors in graphs, Appl. Math. J. Chinese Univ. Ser. A 16 (2001) (4) 385-390.

[613] J. Yu and G. Liu, Binding number and minimum degree conditions for graphs to have fractional factors, J. Shandong Univ., 39 (2004) 1-5 (in Chinese).

[614] J. Yu and G. Liu, Fractional k-factors in graphs, Gongcheng Shuxue Xuebao 22 (2005) (2) 377-380.

[615] J. Yu, G. Liu, M. Ma and B. Cao, A degree condition for graphs to have fractional factors, Adv. Math. (China) 35 (2006) (5) 621-628.

[616] Q. Yu, A note on extendability and factor-criticality, Ann. Comb. 6 (2002) (3) 479-481.

[617] Q. Yu, A note on n-extendable graphs, J. Graph Theory 16 (1992) (4) 349-353.

[618] Q. Yu, Classifying 2-extendable generalized Petersen graphs, Discrete Math. 103 (1992) (2) 209-220. 
[619] Q. Yu, Characterizations of various matching extensions in graphs, Australas. J. Combin. 7 (1993) 55-64.

[620] Q. Yu, On barrier sets of star-factors, Graphs Combin. 6 (1990) (1) 71-76.

[621] Q. Yu, On star factor covered graphs, J. Math. (Wuhan) 11 (1991) (4) 450-454.

[622] Q. Yu, Some theorems on the star-factors, J. Shandong Univ. Nat. Sci. 22 (1987) (4) 24-28.

[623] Q. Yu and Z. Zhang, Extremal properties of $(1, f)$-odd factors in graphs, Ars Combin. 84 (2007) 161-170.

[624] X. Yu, Disjoint paths, planarizing cycles, and spanning walks, Trans. Amer. Math. Soc. 349 (1997) (4) 1333-1358,

[625] J. Yuan, Induced Matching Extendable Graphs - A Survey, (manuscript).

[626] J. Yuan, Induced matching extendable graphs, J. Graph Theory 28 (1998) (4) 203-213.

[627] J. Yuan and F. Yang, NP-Completeness of induced matching problem and CoNP-Completeness of induced matching extendable problem, OR Transactions 4 (2000) 76-80.

[628] C. Q. Zhang and Y. J. Zhu, Factorizations of regular graphs, J. Combin. Theory Ser. B 56 (1992) (1) 74-89.

[629] F. Zhang and H. Zhang, Construction for bicritical graphs and $k$-extendable bipartite graphs, Discrete Math. 306 (2006) (13) 1415-1423.

[630] H. Zhang and F. Zhang, Plane elementary bipartite graphs, Discrete Appl. Math. 105 (2000) (1-3) 291-311.

[631] L. Zhang, Factors and fractional factors of graphs and tree graphs, Ph. D. Thesis, Shandong University, 200111-38 (in Chinese).

[632] L. Zhang and G. Liu, Fractional k-factors of graphs, J. Systems Sci. Math. Sci. 21 (2001) (1) 88-92.

[633] Z. Zhang, Equivalence between $k$-extendable graphs and $n$-factor-critical graphs and their properties, Ph. D. Thesis, Zhongshan University, China, 2008.

[634] Z. Zhang, Y. Li, D. Lou, $M$-alternating Hamilton cycles and $M$-alternating Hamilton paths, (submitted).

[635] Z. Zhang and D. Lou, Bipartite graphs with a perfect matching and digraphs, Austra. J. Combin. (to appear). 
[636] Z. Zhang, D. Lou and X. Zhang, Notes on factor-criticality, extendibility and independence number, Ars Combin. 87 (2008) 139-146.

[637] Z. Zhang, T. Wang and D. Lou, Equivalence between extendability and factorcriticality, Ars Combin. 85 (2007) 279-285. 